\title{
Modular Manipulator For Robotics Applications
}

\section{FINAL REPORT}

(May 1998 - July 2001)

Submitted By:

Program Manager:

Stephen Grupinski

E-Mail: stevegru@armautomation.com

Principal Investigator:

Joseph W. Geisinger, Ph.D.

E-Mail: joewg@armautomation.com

ARM Automation, Inc.

14141 West Highway 290, Suite 700

Austin, TX 78737

Phone Number: (512) 894-3534

FAX Number: (512) 858-2518

Submitted To:

COR: Vijendra Kothari

E-Mail: VIJENDRA.KOTHARI@fetc.doe.gov

U. S. Department of Energy

Federal Energy Technology Center

P.O. Box 880

3610 Collins Ferry Road

Morgantown, West Virginia 26507-0880

For:

Work Performed Under DOE Contract:

DE-AC26-98FT40371

July 2001 


\section{DISCLAIMER}

This report was prepared as an account of work sponsored by an agency of the United States Government. Neither the United States Government nor any agency thereof, nor any of their employees, makes any warranty, express or implied, or assumes any legal responsibility for the accuracy, completeness, or usefulness of any information, apparatus, product, or process disclosed, or represents that its use would not infringe privately owned rights. Reference herein to any specific commercial product, process, or service by trade name, trademark, manufacturer, or otherwise does not necessarily constitute or imply its endorsement, recommendation, or favoring by the United States Government or any agency thereof. The views and options of authors expressed herein do not necessarily state or reflect those of the United States Government or any agency thereof. 


\begin{abstract}
ARM Automation, Inc. is developing a framework of modular actuators that can address the DOE's wide range of robotics needs. The objective of this effort is to demonstrate the effectiveness of this technology by constructing a manipulator from these actuators within a glovebox for Automated Plutonium Processing (APP). At the end of the project, the system of actuators was used to construct several different manipulator configurations, which accommodate common glovebox tasks such as repackaging. The modular nature and quickconnects of this system simplify installation into "hot" boxes and any potential modifications or repair therein.

This work focused on the development of self-contained robotic actuator modules including the embedded electronic controls for the purpose of building a manipulator system. Both of the actuators developed under this project contain the control electronics, sensors, motor, gear train, wiring, system communications and mechanical interfaces of a complete robotics servo device. Test actuators and accompanying DISC ${ }^{\mathrm{TM}_{\mathrm{S}}}$ underwent validation testing at The University of Texas at Austin and ARM Automation, Inc. following final design and fabrication. The system also included custom links, an umbilical cord, an open architecture PC-based system controller, and operational software that permitted integration into a completely functional robotic manipulator system. The open architecture on which this system is based avoids proprietary interfaces and communication protocols which only serve to limit the capabilities and flexibility of automation equipment.

The system was integrated and tested in the contractor's facility for intended performance and operations. The manipulator was tested using the full-scale equipment and process mock-ups. The project produced a practical and operational system including a quantitative evaluation of its performance and cost.
\end{abstract}




\section{TABLE OF CONTENTS}

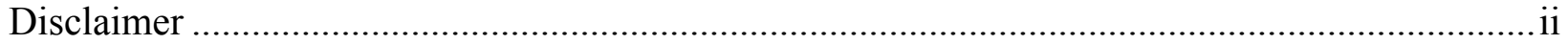

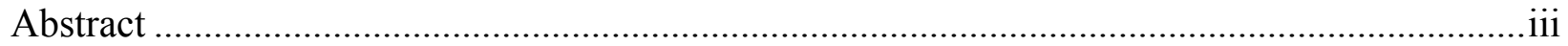

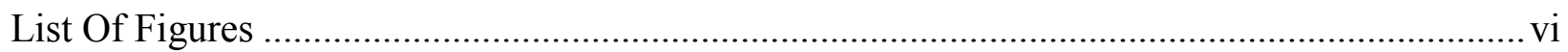

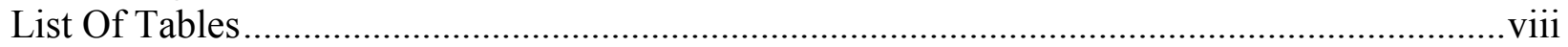

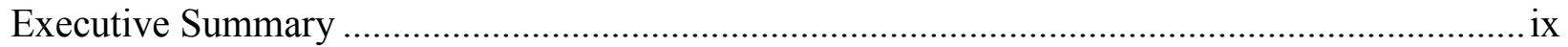

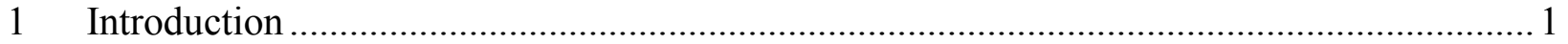

$1.1 \quad$ Project Background .......................................................................................... 2

$1.2 \quad$ Project Objectives ............................................................................................ 4

1.3 Evaluation Of State-Of-The-Art Manipulators And DOE's EM Automation Needs ..... 5

1.3.1 Automated Plutonium Processing and Glovebox Automation Survey ................. 7

1.3.2 Plutonium Processing Characteristics And Glovebox Automation Needs ............ 7

1.3.3 Developed Glovebox Automation Systems ................................................. 8

1.3.4 Glovebox Manipulator And Actuator Requirements ..................................... 11

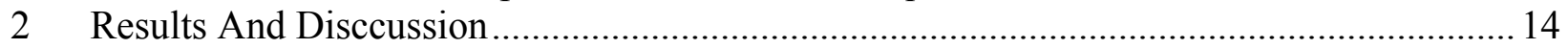

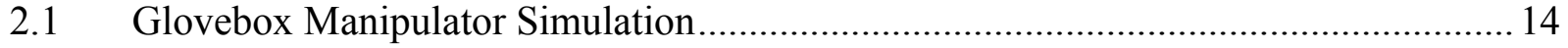

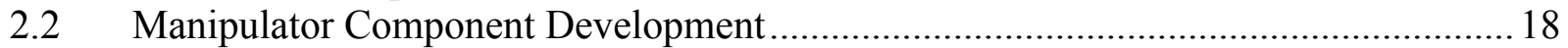

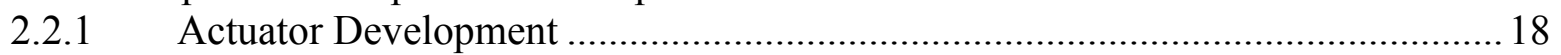

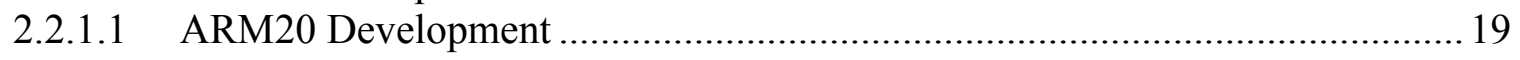

2.2.1.1.1 ARM20 Mechanical Development..................................................... 19

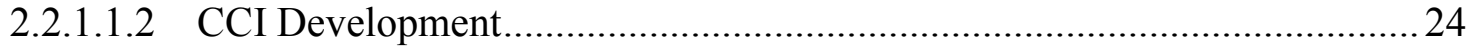

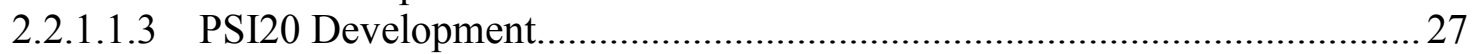

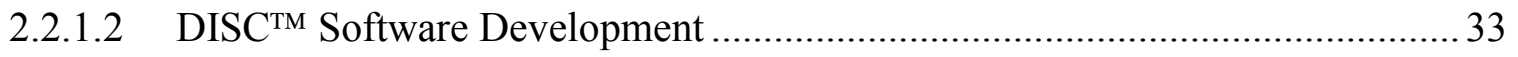

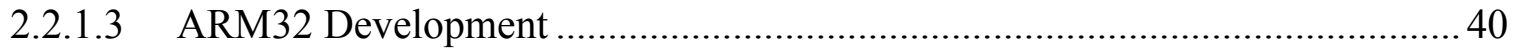

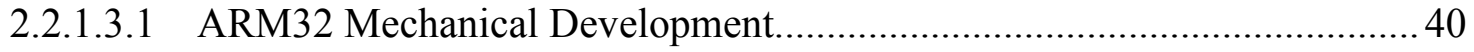

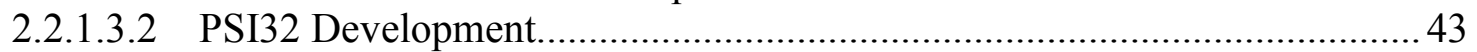

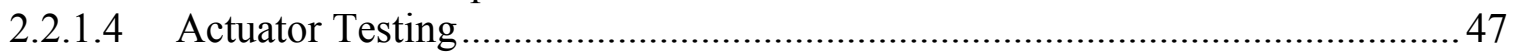

2.2.2 Remaining Manipulator Components ............................................................52

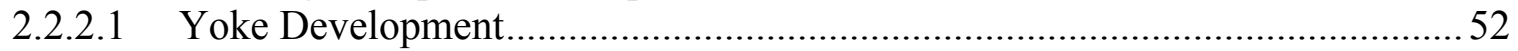

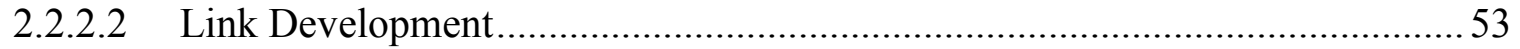

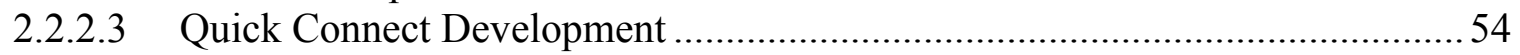

2.2.2.4 Base And End-Effector Plate Development................................................55

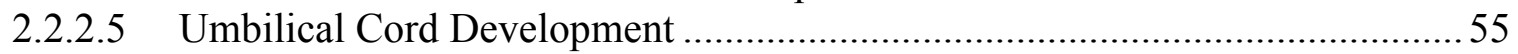

2.2.3 Integrated Power Supply And System Controller .......................................... 56

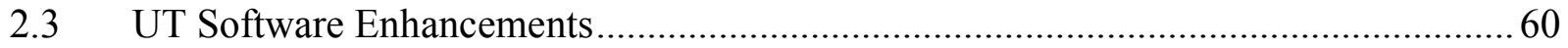

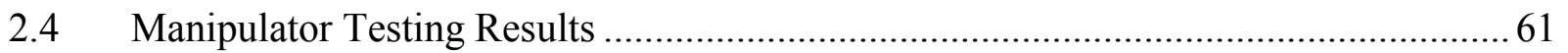

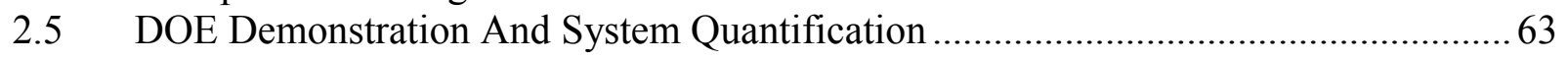

2.5.1 Assembly Of Manipulator Using Quick Connects........................................... 63

2.5.2 Assembly of a Convenience Can Inside a Primary Can......................................6 65

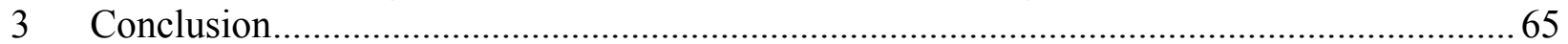

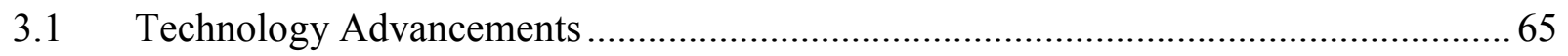

3.2 DOE Commercial Viability Of Modular Robotics ............................................... 68

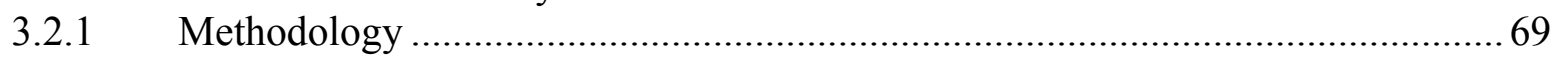

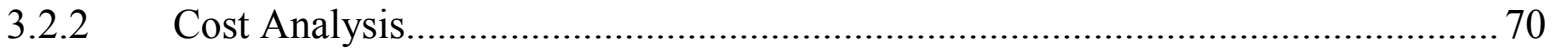

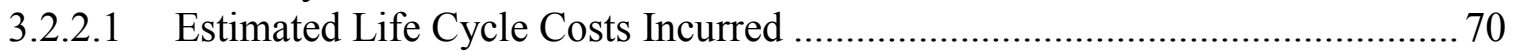

3.2.2.2 Estimated Cost Reduction ............................................................... 70 


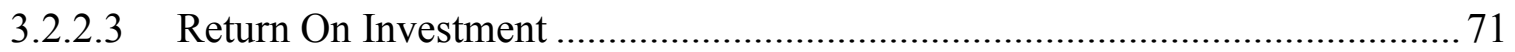

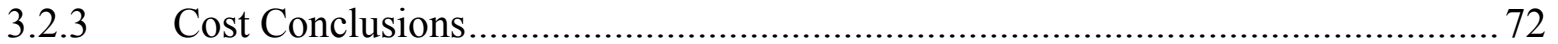

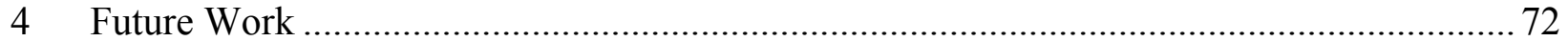

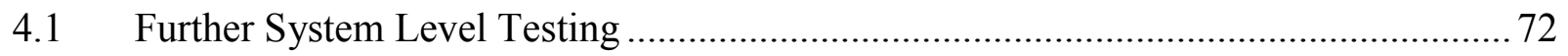

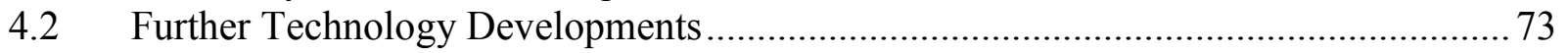

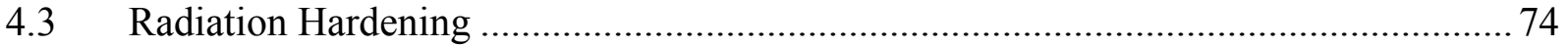

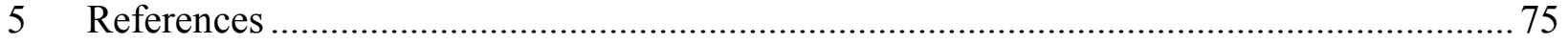

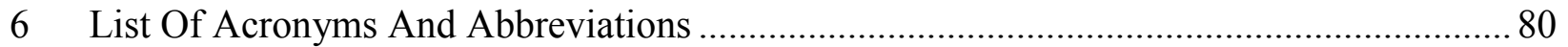




\section{LIST OF FIGURES}

Figure 1: SRS PIP's stage 3 material handling system's conceptual design. 10

Figure 2: Two LR-Mates operational in an ARIES glovebox (left). LR-Mate viewed from "hot"

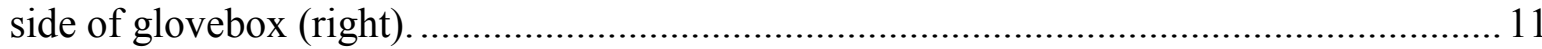

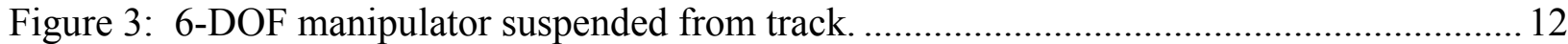

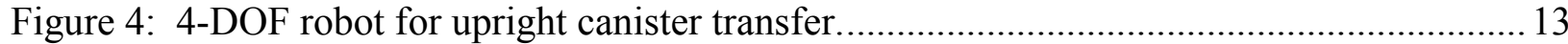

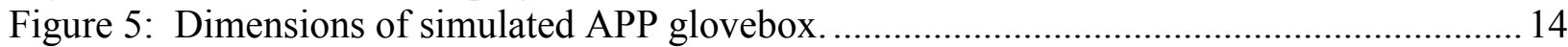

Figure 6: Graphic simulation model. The triangle was placed nearly in line with actuator one's

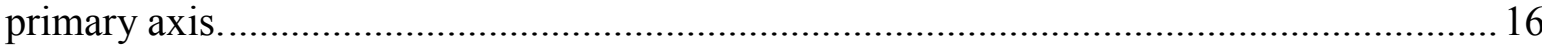

Figure 7: Model of 4 DOF glovebox manipulator. .............................................................. 18

Figure 8: Motor commutation diagrams for a) an MFM motor [MFM, 1998] and b) a Parker

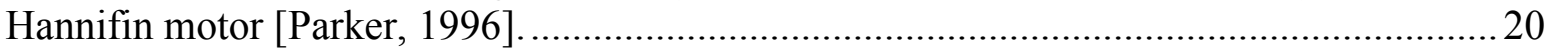

Figure 9: Assembled ARM20 actuator module................................................................... 23

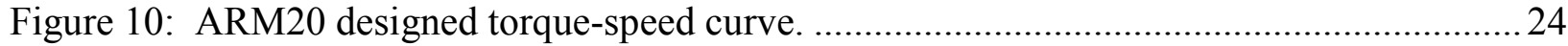

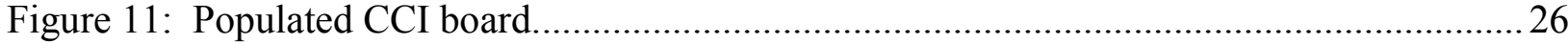

Figure 12: PSI break out board (left) and CCI daughter board interface board (right)............. 27

Figure 13: PSI20 thermistor circuit output voltage versus temperature.................................. 28

Figure 14: Theoretical design curve for motor RTD circuit. .............................................29

Figure 15: Designed Input motor current versus output voltage curve..................................29

Figure 16: Empirical curve for input bus voltage versus output voltage. ............................... 30

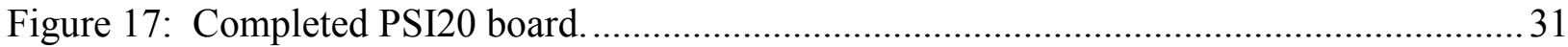

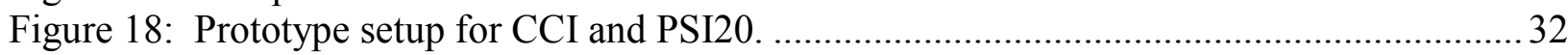

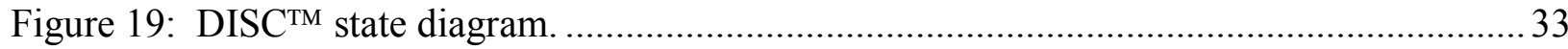

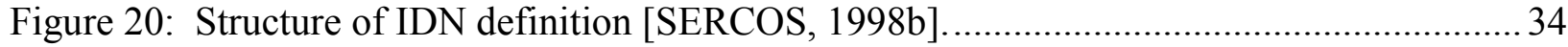

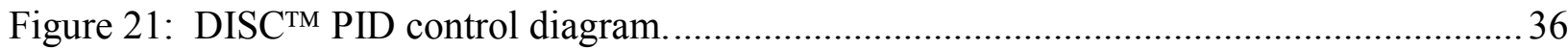

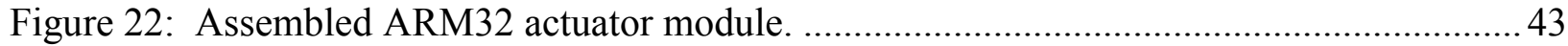

Figure 23: ARM32 designed torque-speed curve. ........................................................ 44

Figure 24: Designed input motor current versus output voltage curve ...................................45

Figure 25: PSI32 empirical curve for input bus voltage versus output voltage........................ 46

Figure 26: Completed PSI32 mounted in housing. .............................................................. 47

Figure 27: Laboratory test assembly and ARM20 actuator. ................................................ 48

Figure 28: ARM20 rotary actuator torque vs. speed performance curve................................49

Figure 29: ARM32 measured torque-speed characteristics. ............................................ 50

Figure 30: ARM20 repeatability and accuracy testing results. ......................................... 51

Figure 31: Yoke32 pieces: base plate (top left), end plate (top center), saddle (top right) and

output extension (bottom left) and assembled Yoke20 output with a quick connect. ......... 52

Figure 32: LINK20 flange (top left), LINK32-20 adapter plate (Top Center), LINK32 flange

(Top Right) and an assembled LINK32 with Yoke32 plates (bottom).............................53

Figure 33: Typical Voss V-Band (left) and application to modular robotics (right). .................54

Figure 34: ARM's custom quick connect assemble............................................................55

Figure 35: Front and rear panels of power supply. ......................................................5 56

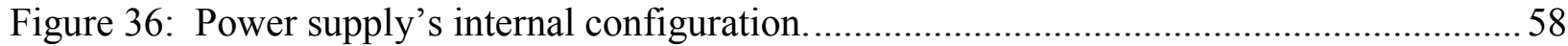

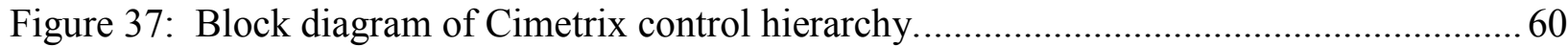

Figure 38: Manipulator configuration \# 1(a) and configuration \#2 (b)................................... 62

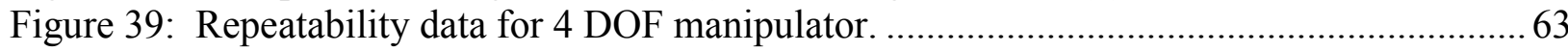

Figure 40: Assembly sequene of the smaller 4 DOF manipulator using gloves........................ 64 
Figure 41: Large 4 DOF modular manipulator assembly sequence.......

Figure 42: Insertion of MOX (lft) into convenience can (ctr) and primary can (rt) [MD, 2001]. 65

Figure 43: Smaller modular manipulator assembling Plutonium canisters...............................66

Figure 44: Larger modular manipulator assembling Plutonium canisters. ............................. 67

Figure 45: Various manipulator configurations comprised of ARM32 and ARM20 modules.... 68 


\section{LIST OF TABLES}

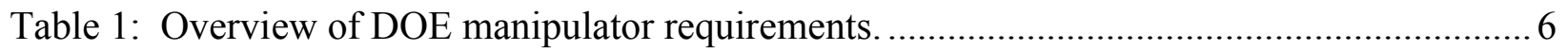

Table 2: FANUC LR-Mate 100i specifications [FANUC, 1993] .......................................... 11

Table 3: UTRRG APP glovebox manipulator parameters. ................................................... 15

Table 4: Simulation RMS values (values given in $\mathrm{N}$ for force and $\mathrm{N} \cdot \mathrm{m}$ for torque)................. 17

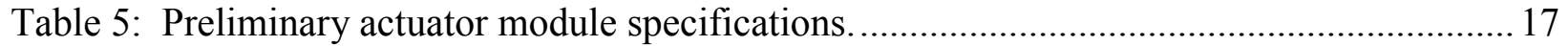

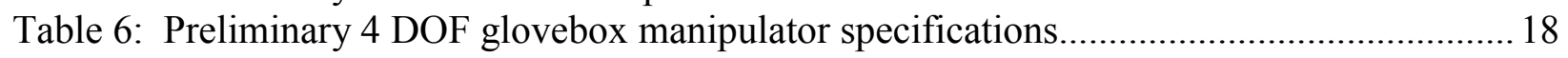

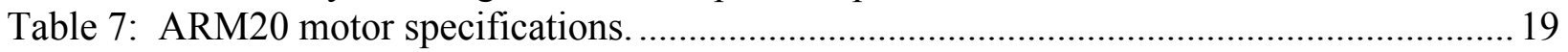

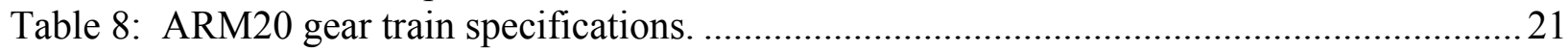

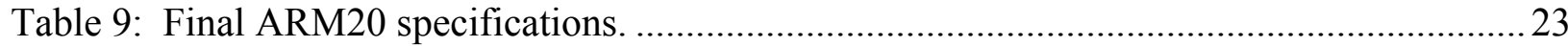

Table 10: Comparison of CCI's preliminary specifications to its final specifications................27

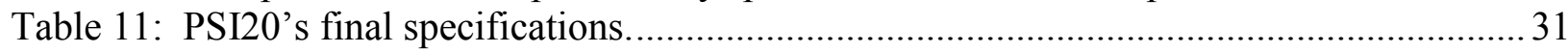

Table 12: DISC ${ }^{\mathrm{TM}}$ error numbers (in hexadecimal) associated with IDN 129......................... 37

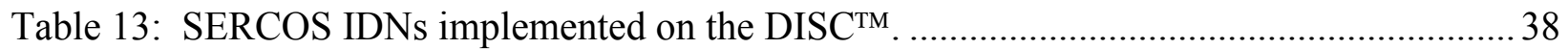

Table 14: SERCOS IDNs implemented on the DISC ${ }^{\text {TM }}$ (continued from Table 13)...................39

Table 15: Minimum IDN setup list required for DISC ${ }^{\mathrm{TM}}$ operation. ...................................... 40

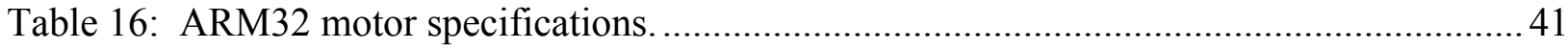

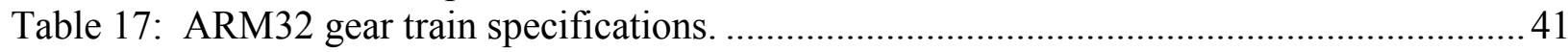

Table 18: Final ARM32 specifications. ............................................................................ 43

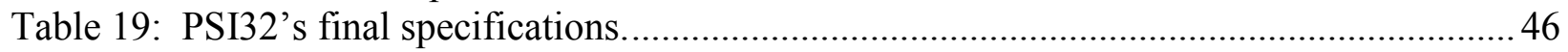

Table 20: Actuator component temperature limitations used during test. .............................. 49

Table 21: Pin assignment for power supply's rear panel connectors....................................5 57

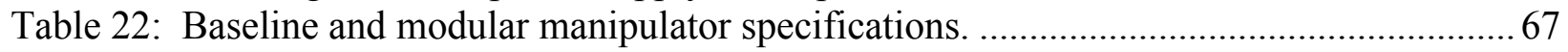

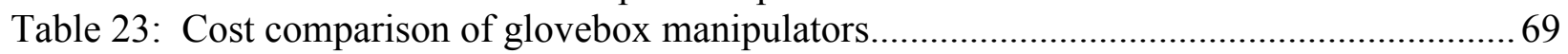

Table 24: Estimated cost reduction for the baseline automation implementations.....................71

Table 25: Estimated Return On Investment for the baseline automation implementations......... 72 


\section{EXECUTIVE SUMMARY}

The Department of Energy (DOE) Environment Management (EM) program has the mission of cleaning up a legacy of more than 50 years of nuclear operations and weapons production. A great number of the EM tasks involve the monitoring, characterization, cleanup, and decommissioning of facilities, which are contaminated with toxic and/or radioactive materials. Often these activities require or benefit from the use of robotic and tele-robotic systems in lieu of human labor. These dexterous systems serve in many capacities in order to limit exposure of personnel to the hazards associated with such an environment. If the incredible volume of hazardous materials, which exists at DOE facilities, is to be reduced within the next decade, all resources allocated to contend with these tasks must provide maximum efficiency. Given the number of complex tasks which need automation, all robotic systems applied must provide a maximum of reliability and versatility.

Each DOE application contains a highly unique set of task requirements, and therefore, requires an entirely different kinematic system. Traditional robotic solutions, with their low payload-to-weight ratios, proprietary system controls, small work volumes, and high integration costs, offer little in the way of customizability to meet the DOE's specific needs. This places the DOE in the position of either commissioning the development and construction of an entirely custom robotic system for each task, or purchasing existing industrial robot systems and paying the high price of catering to their many limitations.

To meet the goals of the DOE's EM Ten-Year Plan, a number of advances must be made in the field of robotic systems. The DOE requires technology advances that reduce total life cycle automation expenses. These systems must include features that represents a standard for reducing training and deployment risks; are quickly repairable/replaceable at an affordable cost; increase a shared use of technology to defer multiple development costs; generate a minimum of secondary waste material; allow rapid design, prototyping, and deployment of custom automation solutions, technology; are compatible with emerging standards in open architecture communication and control; and are provided by a reliable American supplier. In short, the ideal DOE robotics technology frees DOE development resources to allow its automation experts to concentrate their efforts and budgets on solving the real EM remediation problems

This project developed modular robotic manipulator technology that addresses many of these DOE technology needs. The application targeted to demonstrate this technology was glovebox automation associated with Automated Plutonium Processing (APP). The modular architecture and quick-connects of this system simplify installations and any potential modifications or repair in "hot" gloveboxes. Key arguments for employing a modular approach are shorter design timelines, simplified repair and reduced life cycle costs from a technology, which can be applied to multiple automation tasks within the DOE complex.

While the project concentrated on designing a modular manipulator for APP, a review was undertaken to understand the needs of a wide range of DOE applications to ensure that the module designs will be applicable to DOE tasks other than APP. From this review, ARM produced a comprehensive overview of the automation requirements of key DOE applications within the context of modular automation. The resulting topical report outlined a roadmap of how modular robotic systems can be applied to DOE automation applications. This study encompassed a review of literature, telephone conversations with automation experts and on site visits within the DOE complex. 
The review of DOE automation needs was followed by the in-depth design and fabrication of components for a modular manipulator tailored for glovebox automation. The study and simulation of manipulators in a glovebox environment set the constraints on the design of the components. The requirements were acquired from four dedicated glovebox processing systems that were developed for handling and packaging the complex's nuclear materials: one developed by LLNL for processing tritium in a glovebox, one developed by BNFL, Inc. for Plutonium Stabilization and Packaging Systems (PuSPS) at RFETS, and two systems developed in response to the initiatives set forth by START I and START II, one by SRS, and one by LANL. The components designed included two sizes of actuator modules (the ARM20 and ARM32 models capable of producing 47 and $130 \mathrm{~N}$-m output torque, respectively), two sizes of links and yokes to mate with their respective actuators, one size of quick-connects, umbilical cord, base plate, end effector plate, power supply, and system controller. The design of the actuators encompassed mechanical, electrical, and software engineering in the development and fabrication of an unusually compact Digital Intelligent Servo Amplifier (DISC ${ }^{\mathrm{TM}}$ ), and the mechanical components for each actuator. The system was then integrated and tested at ARM's facility for intended performance and operations. The manipulator was tested using the full-scale equipment and process mock-ups. The complete testing effort, including the testing of the actuators, produced a quantitative evaluation of the manipulator's performance.

In conjunction with the manipulator component design effort, UT developed obstacle avoidance and manual controller software. The obstacle avoidance software was designed to keep the manipulator at a safe distance from the glovebox and any obstacles within the glovebox. This is necessary function for tele-operation and path planning purposes. The manual controller software was an enhancement to the Cimetrix system controller software utilized for the system controller. This software allows the manipulator to be taught points after it has been assembled inside the glovebox.

The baseline technology used for comparison purposes is a Fanuc LR-Mate 100i used by LANL in ARIES and proposed for use by SRS in the PIP's stage 3 material handling system. ARM's modular robotic technology has made the following significant advantages over the baseline:

- Increased net payload to weight ratio from approx. 1:11 to 1:3 with an increase in maximum wrist payload from $3 \mathrm{~kg}$ to $12 \mathrm{~kg}$ for the same reach;

- Reduced the umbilical cord from two 50-wire cables to a single 12-wire cable;

- Demonstrated at least 5 custom configurations using the modular technology;

- The modules are a repairable unit that can easily be bagged in or out of a glovebox whereas the LR-Mate has to be considered a disposable unit of upon failure;

- The modular robot can be calibrated, broken down, passed through a glovebox port and reconfigured inside an existing glovebox to within a demonstrated accuracy of $0.5 \mathrm{~mm}$.

The testing results verified the initial projection of its benefits applied to DOE's automation needs. Furthermore, the relevancy of the technology to DOE has not waned, as many of the STCG needs prevalent in 1998 still have not yet been met and more needs have surfaced as acceptable personnel radiation dose levels have declined. The ability to introduce the technology into existing gloveboxes addresses many needs previously untouched in the site clean up and nuclear material handling realm where existing solutions are either prohibitively expensive or pose significant health and safety risks to personnel. The bottom line is that this technology yields a total life cycle cost savings of at least $\$ 100,000$ over the baseline commercial robotic technology allowing the DOE to address many of the site closure needs that exist today. 


\section{INTRODUCTION}

The Department of Energy (DOE) program has the mission of cleaning up a legacy of more than 50 years of nuclear operations and weapons production. As originally laid out in [DOE, 1998a], the DOE envisions completing a majority of the clean-up effort by 2006 . In order to meet this mission, The DOE established five Environmental Management (EM) program focus areas which are managed by the Office of Science and Technology (OST) [OST, 1999]: Deactivation \& Decommissioning (D\&D), Nuclear Materials (NM), Subsurface Contaminants, Tanks, and Mixed Waste (MW). There exist four crosscutting programs whose mission is to develop technology to support and complement each of the focus areas: Characterization, Monitoring \& Sensor Technology (CMST), Efficient Separations and Processing (ESP), Industry Programs (IP), and Robotics. While this project operated under the D\&D focus area, the technology pertains to many applications in the EM program.

This report presents the development of a modular robotic manipulator designed for glovebox operations. The application targeted to demonstrate this technology was Automated Plutonium Processing (APP). This effort came about in response to a proposal submitted early in 1997 which outlined an approach for utilizing a system of five intelligent actuator module sizes to construct two manipulators: one tailored to the needs of D\&D and the other to APP [ARM, 1997]. Key arguments for employing a modular approach were shorter design timelines, simplified repair and reduced life cycle costs from a technology, which can be applied to multiple automation tasks within the DOE complex.

While the focus of the project was on designing a modular manipulator for APP, a review was initially undertaken to examine the needs of the other DOE applications to ensure that the module designs will be applicable to other tasks. Thus, the first objective of this project was to conduct a comprehensive overview of the automation requirements of key DOE applications within the context of modular automation. As such, this report outlined a roadmap of how modular robotic systems can be applied to DOE automation applications. An in-depth study, of all the DOE's automation needs, was deemed impossible; however, an overview, which provides sufficient information to facilitate the design of a versatile set of actuator designs, has been achieved. This was accomplished by a review of literature, telephone conversations with automation experts and on site visits within the DOE complex.

The review of DOE automation needs was followed by the in-depth design and fabrication of the components for a modular manipulator targeted towards glovebox automation. The constraints on the design of the components were set by the study. The components designed included two sizes of actuator modules, two sizes of links with quick-connects, yokes, umbilical cord, power supply, and system controller. The University of Texas at Austin (UT) performed the testing of the actuators under this task to verify the design parameters.

In conjunction with the manipulator component design effort, UT developed obstacle avoidance and manual controller software. The obstacle avoidance software was designed to keep the manipulator at a safe distance from the glovebox and any obstacles within the glovebox. This is necessary for tele-operation and path planning purposes. The manual controller software was an enhancement to the Cimetrix system controller software utilized by ARM for the system controller. This software allows the manipulator to be taught points after it has been assembled inside the glovebox. 
The system was then integrated and tested at ARM's facility for intended performance and operations. The manipulator was tested using the full-scale equipment and process mock-ups. The complete testing effort, including the testing of the actuators at UT, produced a quantitative evaluation of the manipulator's performance.

\subsection{Project Background}

A great number of the EM tasks involve the observation, characterization, cleanup, and decommissioning of facilities, which are contaminated with toxic and/or radioactive materials. Often these activities either require or justify the use of robotic and tele-robotic systems in lieu of human labor. These robotic systems serve in many capacities in order to limit exposure of personnel to the hazards associated with such an environment. In some cases, robotic solutions have been attempted through the use of traditional robotic technology [Hollen and Rzeszutko, 1997], [HP, 1994] \& [Noakes, et al, 1997]. While some of these systems have enjoyed moderate success when built to the task, like the SPAR Modified Light Duty Utility Arm (MLUDA) [Burks, et al, 1997], others have failed to demonstrate the desired feasibility [Dennison, et al, $1995]$.

Generic off-the-shelf robotics technology has proven to be limited in its ability to meet the diverse requirements of the DOE. Outside of hydraulic manipulators, the DOE has found commercial robotics to be technology deficient in its desire to automate such a wide range of unique applications. The following list summarizes some of the predominant characteristics that the DOE seeks over commercially available technology:

\section{Performance:}

- Increased net payload to weight ratio (less than 1:10) [Bajura, 2001]

- Smaller umbilical cords to allow portability/remote deployment [GNET, 1998]

- Capacity for customization [Tibrea and Maddux, 1998]

- Easily repairable or replaceable components [Tibrea and Maddux, 1998]

- Reconfigurable for new or changing applications [NMFA, 2000c]

Cost:

- Reduce worker exposure [DOE, 2000b]

- Lower integration cost [EM, 2000]

- Training which spans multiple systems through reuse [GNET, 1998]

- Lower repair and maintenance costs [NMFA, 2000b]

- Decreased disposal cost [EM, 2000]

- Lower design, testing, prototyping, and manufacturing costs as compared to custom robots [DOE, 1998b]

- Decreased lead times with unrealized productivity (Custom Robots) [NMFA, 2000b]

- Increased cost sharing between developed technologies [DOE, 1998b]

The low payload-to-weight ratio of existing robotics technology presents a major problem for systems, which must be made quickly and easily deployable on-site by crane, lift, or hand. The standard electric robot manipulator weighs roughly $20 \mathrm{~kg}$ for every $1 \mathrm{~kg}$ of payload capacity. When system controllers and amplifiers are added the payload to weight ratio jumps to nearly 1 to 40 . In applications where the required payload is $45 \mathrm{~kg}\left(100 \mathrm{lb}_{\mathrm{m}}\right)$ or more, as in D\&D work, conventional electric robot systems weigh in at more than $1800 \mathrm{~kg}\left(4000 \mathrm{lb}_{\mathrm{m}}\right)$. Hydraulic robots, on the other hand, offer higher payload-to-weight ratios [Schilling, 1999]. However, the low weight of these systems is deceptive because they require the close support of large 
hydraulic umbilicals, which rely on a system of motors, compressors, filters, valves, and electronic controls. These same hydraulic systems also add considerable risk to the application, as they are prone to substantial fluid spills into contaminated areas as a result of a failure or leakage. This leaves DOE facilities without a viable robotics option for a portable robotic manipulator.

The large size of existing robotics technology is not the only problem that faces EM automation. In most DOE applications, space is limited. Whether the application is inside a retired reactor facility or a tightly sealed analysis chamber such as a glovebox, any manipulator must occupy as little space as possible while providing the highest degree of reach and dexterity. Conventional robotic manipulators are incapable of high ranges of motion in most joints. This limits their dexterity and capabilities. In order to successfully carry out complex tasks such as sorting materials, manipulating power tools, and reaching around objects, a more refined and dexterous manipulator technology is needed.

If the incredible volume of hazardous materials, which exists at DOE facilities, is to be processed within the next decade, all resources allocated to contend with these tasks must provide a maximum of efficiency. Given the number of complex tasks which need automation, all robotic systems applied must provide a maximum of reliability and "up-time". In the event of a process change or eventual component failure, processes must be halted and the monolithic manipulator systems must either be entirely replaced or repaired at great expense and loss of time. This type of event consumes days at a time for each manipulator and expends valuable human resources as well. Measures must be taken to provide robotic systems, which are quickly and easily repairable or simply modified, on demand, as processes change.

Their high price tags, integration costs, and extensive operational expenses parallel the limitations of available robotic systems. Additionally, each DOE application contains a highly unique set of task requirements, and therefore, requires an entirely different manipulator system than industrial applications. Traditional robotics solutions, with their low payload-to-weight ratios, proprietary system controls, small work volumes, and high integration costs, offer little in the way of customizability to meet the DOE's specific needs. This places the DOE in the position of either commissioning the development and construction of entirely custom robotics technology for each task, or purchasing existing industrial robot systems and paying the high price of catering to their many limitations.

The design of custom robotic systems is both costly and time consuming. The complex task of designing robotic systems calls for personnel with expertise in several fields. These personnel must then decide how to translate task specifications into robotic parameters. These robot specifications can then be used to specify joint actuator criteria. In turn, actuator specifications lead to the selection of a range of component technologies, which must be integrated into robotic actuators and then into a full system. Additionally, power supplies, amplifiers, and motion control hardware must be matched to the robotic design. Finally, some form of software, which must be configured for the system on hand, must control all of these components. Once the unavoidable bugs are worked out of such a custom robotic system, the robot must then be tested and certified before its true performance can be known. This entire process can take years while consuming entire project budgets.

The use of commercial manipulator systems in unique situations is equally fraught with expense. Industrial robots are designed to perform specific sets of tasks under well-controlled conditions. Therefore, considerable expense must be put forth to adapt these robots to tasks 
which are outside this regime. Standard industrial robots typically require 3-5 times their initial cost for adaptation to these tasks due to their size, masses of cable, monolithic interfaces, proprietary system controls, and limited performance characteristics [Evans, 2000]. This high integration cost becomes compounded due to their operation in a radioactive environment. Their low payload-to-weight ratio requires that any mobile or portable deployment vehicle be sized accordingly and, thereby, cost substantially more than would be necessary with a lighter manipulator.

The operation, maintenance, and disposal of industrial robots in DOE applications presents a cost factor which can greatly exceed the initial cost of installation and integration. These large and monolithic machines cannot be easily repaired or serviced in the event of a failure because their entire structure must be removed from the contaminated site before being serviced. Once contaminated, their large singular structure is difficult to disassemble and package for disposal further driving up cost.

In order to meet the goals of the Ten-Year Plan of the DOE's EM program, a number of advances must be made in the field of robotic systems. The following list summarizes the required advances/needs of the DOE ([RIM, 2000] and [EM, 1998]):

- Technology which reduces total automation expense

- Systems which are quickly and affordably repairable/replaceable

- Standards in robot technology for reduced training and deployment risks

- Commonality between robotic systems to increase shared use of technology and to defer multiple development costs

- Robotics which generate a minimum of secondary waste material

- Technology which allows rapid design, prototyping, and deployment of custom automation solutions

- Robotics technology which is compatible with emerging standards in open architecture communication and control

- Accessibility to a reliable commercial supplier of robotics technology

In short, the DOE requires a robotics technology, which frees DOE development to allow its automation experts to concentrate their efforts and budgets on solving the real EM remediation problems

\subsection{Project Objectives}

The objective of this effort is to develop a modular robotic manipulator technology for DOE EM operations, which addresses many of the issues discussed in the previous section. For this project, the manipulator is targeted for Automated Plutonium Processing (APP) glovebox operations. The manipulator system has the capability of custom configurations, which accommodate common glovebox tasks such as materials repackaging. The modular nature and quick connects of this system simplify installations into "hot" boxes and any potential modifications or repair therein.

This work focused on the development of self-contained robotic actuator modules incorporating the DISC ${ }^{\text {TM }}$ (Digital Intelligent Servo Controller) for the purpose of building a manipulator system. Each actuator module integrates the control electronics, sensors, motor, gear train, wiring, system communications, and electrical and mechanical interfaces to form a complete robotic servo device. Test actuators and accompanying DISC ${ }^{\mathbf{T M}_{\mathbf{S}}}$ underwent validation testing following final design and fabrication. The system also included custom designed links, 
an umbilical cord, an open architecture PC-based system controller, and operational software that permitted integration into a completely functional robotics manipulator system. The open architecture on which this system is based eliminates proprietary interfaces and communications, which only serve to limit the capabilities and flexibility of automation equipment.

This project was broken down into five tasks. For the first task, ARM evaluated the State-of-the-Art manipulators along with the perceived needs for DOE's five EM focus areas [Black, 1998]. The results from this study were used to define the manipulator specifications for the task of developing the manipulator's components. UT, in parallel with ARM's development, simulated the proposed APP manipulator to determine the torque and speed requirements of the actuators. In addition to this simulation, UT also enhanced the software for the system controller by integrating an obstacle avoidance routine in the system controller. They also interfaced a manual controller to the system controller to allow for tele-operation. All aspects of the manipulator component development were then brought together at ARM's facility where the manipulator was debugged and its functionality verified.

\subsection{Evaluation Of State-Of-The-Art Manipulators And DOE's EM Automation Needs}

The D\&D Focus Area strives "to develop and demonstrate improved technologies and systems to solve customer-identified needs to characterize, deactivate, survey and maintain, decontaminate, dismantle, and dispose of DOE surplus facilities and their contents, and to facilitate the acceptance, approval, transfer, commercialization and implementation of these technologies and systems" [DDFA, 2000a]. In an effort to follow this mission, a major objective of this project is to demonstrate the viability of modular robotics technology applied to EM applications, in general, and the D\&D Focus Area, in particular. Thus, this project's goal is to show that modular robotics is an improved automation technology that addresses the needs of the EM focus areas. In order to demonstrate this, ARM researched the state-of-the-art technology in modular manipulators through activities, including but not limited to, a literature review and discussions with DOE and industry personnel. ARM also visited several DOE sites involved in site remediation and robotics system development regarding the technology issues related to remote operations. This effort allowed ARM to gain insight into the DOE's EM automation requirements. A Topical Report presenting these findings was prepared at the end of this evaluation [Black, 1998]. In addition to this study, ARM gathered information associated with plutonium glovebox operations including forms of other types of automation systems used for alternative APP tele-operator architectures. Based on this information, ARM established a baseline for comparing the modular manipulator system against the present day practices. The results of this effort are summarized herein.

The DOE has considered the use of robotics for a variety of tasks in each of the focus areas including NM [ARIES, 2000], MW [EPE, 2000], D\&D [Noakes, et al, 1997], and Tank Waste Retrieval (TWR) [Burks, et al, 1997]. Based upon the review of each of the focus areas automation applications by ARM, a set of approximate manipulator requirements was developed and summarized in [Black, 1998] and repeated in Table 1. Some general comments regarding these general specifications are as follows: A key factor in selecting the accuracy and repeatability specifications is operation mode. Tele-operated systems are the least demanding because of the adaptability of the human operator. Teach-pendant taught systems do not require much accuracy, but demand repeatability to ensure points need not be re-taught (a tedious 


\begin{tabular}{|c|c|c|c|c|}
\hline & APP & D\&D & NM & MWO \\
\hline \multicolumn{5}{|c|}{ Manipulator Specifications } \\
\hline Reach [m (in)] & $0.6(24)$ to $0.8(32)$ & $1.5(59)$ & $0.6(24)$ & $1(39)$ \\
\hline Payload [kg $\left.\left(\mathrm{l} \mathrm{lb}_{\mathrm{m}}\right)\right]$ & $8(18)$ to $10(22)$ & $90(198)$ & $0.5(1.1)$ to $2(4.4)$ & $90(198)$ \\
\hline $\begin{array}{l}\text { Arm Degrees of } \\
\text { Freedom }\end{array}$ & 4 to 6 & $6 \times 2$ arms & 4 to 6 & 6 \\
\hline $\begin{array}{l}\text { Tele-operated, } \\
\text { Teach-pendant } \\
\text { Playback, Off-line }\end{array}$ & $\begin{array}{l}\text { Initially teach-pendant } \\
\text { Tele and off-line poss. }\end{array}$ & Tele-operated & Teach-pendant. & $\begin{array}{c}\text { Primarily Tele- } \\
\text { operated }\end{array}$ \\
\hline \begin{tabular}{|l} 
Accuracy I \\
Repeatability \\
$+/-[\mathrm{mm}$ (in)]
\end{tabular} & $\begin{array}{c}1(0.040) \\
0.1(0.004)\end{array}$ & $\begin{array}{c}\mathrm{N} / \mathrm{A} \\
0.5(0.020)\end{array}$ & $\begin{array}{c}0.5(0.020) \\
0.25(0.010)\end{array}$ & $\begin{array}{c}\mathrm{N} / \mathrm{A} \\
0.25(0.01)\end{array}$ \\
\hline Linear Rail Mount & For some tasks & Not typically & Yes & Likely \\
\hline $\begin{array}{l}\text { Speed } \\
{[\mathrm{m} / \mathrm{sec}(\mathrm{in} / \mathrm{sec})]}\end{array}$ & $0.7(28)$ & $0.91(36)$ & $0.7(28)$ & $0.91(36)$ \\
\hline Force Control & No & Yes & No & Yes \\
\hline End Effector Type & Electric gripper & $\begin{array}{c}\text { Mix of electric } \\
\left(110 \mathrm{~V}_{\mathrm{AC}}\right) \text { and } \\
\text { pneumatic }\end{array}$ & $\begin{array}{l}\text { Standard gripper } \\
\text { (electric or air) }\end{array}$ & $\begin{array}{l}\text { Multiple tools } \\
\text { (electric or air) }\end{array}$ \\
\hline Portability & $\begin{array}{c}\text { Pieces must facilitate } \\
\text { bagout }\end{array}$ & Yes & No & $\begin{array}{l}\text { System must } \\
\text { facilitate "hot" } \\
\text { repairs }\end{array}$ \\
\hline $\begin{array}{l}\text { Obstacle } \\
\text { Avoidance }\end{array}$ & Yes, or limited motion & Yes (for autonomy) & $\begin{array}{c}\text { No, highly } \\
\text { structured } \\
\text { environment }\end{array}$ & $\begin{array}{l}\text { Advantageous } \\
\text { (not needed) }\end{array}$ \\
\hline \begin{tabular}{|l|} 
Positive \\
Pressurization \\
\end{tabular} & Yes & Not typically & Not typically & Yes \\
\hline \multicolumn{5}{|c|}{ Operational Conditions } \\
\hline \begin{tabular}{|l} 
Radiation Level \\
[rad/h]
\end{tabular} & $0.3-1$ & $0.1-1000$ 's & $\begin{array}{l}\text { None at present, } \\
\text { Potential exists }\end{array}$ & $0.001-0.01$ \\
\hline $\begin{array}{l}\text { Corrosive/ Hazard } \\
\text { Environment } \\
\end{array}$ & $\begin{array}{c}\text { Yes, } \\
\text { Plutonium dust }\end{array}$ & Yes & None & $\begin{array}{c}\text { Mix of industrial } \\
\text { chemicals }\end{array}$ \\
\hline Confined Space & Yes & Yes & Yes & Slightly \\
\hline Serviceability & Through glove ports & Suited technicians & Suited technicians & $\begin{array}{c}\text { Suited } \\
\text { technicians }\end{array}$ \\
\hline Wet or Spray & No & $\begin{array}{c}\text { Potentially (even } \\
\text { submerged) }\end{array}$ & No & $\begin{array}{l}\text { Potential from } \\
\text { cutting system }\end{array}$ \\
\hline
\end{tabular}

Table 1: Overview of DOE manipulator requirements.

process). The most demanding are off-line programmed CAD driven systems that assume a perfect world model. Both accuracy (the maximum error between expected and actual positions over the entire positional range) and repeatability (the ability of a positioning system to return to a location during operation when approaching the location from the same direction) are required for these systems. Unlike some high-volume industrial production systems, speed is not critical for any of the DOE applications considered; thus, the speed specification serves merely as a guideline. Tank applications were not included in the study's results because they are the least conducive to the modular approach, and are sufficiently unique to be addressed by existing monolithic designs [Kiebel, 1997]. 


\subsubsection{Automated Plutonium Processing and Glovebox Automation Survey}

This project is primarily concerned with APP tasks associated with material handling operations. While these tasks take place both inside and outside radioactively "hot" gloveboxes, the applications addressed herein only apply to those carried out in a glovebox. Glovebox applications exist within all EM Focus Areas (FA) as well as other programs within the DOE (NM [NMFA, 1999], [NMFA, 2000b], [ARIES, 2000]; D\&D [DDFA, 2000b]; MW [EPE, 2000]), Materials Disposition (MD) [RIM, 1998], [Bronson, et al, 1997] and Defense Programs (DP) [DP, 1996]. Many types of glovebox operations, including Plutonium processing and handling operations, are performed within the DOE complex. While many of the applications within DP are classified, discussions with DOE personnel at LANL indicate that this technology is needed to reduce worker exposure and many of the requirements are similar to other glovebox operations. There are many needs within the DOE Complex requiring glovebox automation but the most immediate needs are those summarized in [NMFA, 1999], pgs. 40-42, which call out specific sites (LANL, ANL, HAN, SNL, SRS and RFETS) that require an immediate infusion of glovebox automation in order to meet the DOE's commitment outlined [DOE, 1998a] and reiterated in [Richardson, 2000]. The use of glovebox automation technology will also be critical to the DOE in its efforts to comply with its mandated ALARA principles in handling the hazardous materials associated with the cleanup process.

\subsubsection{Plutonium Processing Characteristics And Glovebox Automation Needs}

The arguments presented in [Black, 1998] limited the scope of Plutonium processing and nuclear material handling to glovebox operations. Two types of Plutonium and other nuclear materials processing categories were presented:

(1) In-box processing and stabilization of residues and segregation of Plutonium laden materials (D\&D, NM and MW applications).

(2) Processing of Plutonium metal and oxides within gloveboxes for weapons production or disposition (DP, and NM applications).

The first category pertains to applications such as chemical and thermal processing, material analysis and packaging. The second application involves the interaction with complex machinery such as such as lathes, furnaces, etc. to transport the nuclear/toxic materials within the glovebox environment.

Operations associated with materials processing in a glovebox are similar to many industrial tasks, but the unique environment and material properties create the following unique set of challenges [McKee, 1998]:

- Plutonium

- Low to moderate levels of ionizing radiation.

- Highly abrasive.

Corrosive.

口 Pyrophoric.

口 Disperses and permeates readily. Diffuses quickly.

- Reaction and behavior with equipment is not well understood.

- Glovebox Environment

- Existing gloveboxes may not be readily altered or even modified at all. 
- Complex mechanical operations for maintenance and repair are difficult or impossible through gloves.

- Failed equipment may not be removed easily or at all. If a broken piece of equipment cannot be bagged-out through a glove port (approximately $216 \mathrm{~mm}$ $\left(8^{1 / 2}{ }^{\prime \prime}\right)$ diameter) it must remain in place. Broken equipment obstructs further operations. If it renders the entire glovebox unusable a significant volume of waste is generated and an expensive system must be disposed of and replaced. A moderate sized glovebox alone costs between $\$ 250,000$ and $\$ 500,000$.

- An equipment malfunction, which penetrates the glovebox and exposes the room to Plutonium or other toxic materials, is catastrophic. In addition to the human exposure issues, cleanup can easily run into the millions of dollars.

\subsubsection{Developed Glovebox Automation Systems}

A review of the literature by ARM for [Black, 1998] did not reveal any previously developed modular robotic systems for glovebox (Plutonium or otherwise) operations. However, at least four dedicated glovebox processing systems have been developed for handling and packaging the complex's nuclear materials. The first one presented below was developed by LLNL for processing tritium in a glovebox. The second one was developed by BNFL, Inc. for Plutonium Stabilization and Packaging Systems (PuSPS) at RFETS [Lyman, 1998] in response to [DOE, 1998a]. The last two systems were developed in response to the initiatives set forth by START I and START II ([Bronson, et al, 1997] \& [Kriikku, et al, 1999]), one by SRS, and one by LANL. These last three systems have been developed in accordance with DOE-STD-3013-96 that utilizes standardized canisters for packaging the nuclear materials.

LLNL has had several demonstration glovebox facilities in operation since April of 1991 [Dennison, et al, 1995]. In August of 1993, LLNL contracted with International Business Machines (IBM) to supply a gantry robot for installation in a Tritium processing glovebox for DP. This large robotic system was installed in a "double-decker" glovebox for handling of large containers. The system has six degrees-of-freedom; a three revolute axis wrist attached to threeaxis linear Cartesian gantry system. The robot is able to reach $1.07 \mathrm{~m}$ (42 in) down, grasp $68 \mathrm{~kg}$ $\left(150 \mathrm{lb}_{\mathrm{m}}\right)$ objects, and traverse $7.2 \mathrm{~m}(282 \mathrm{in})$ in $\mathrm{X}, 0.69 \mathrm{~m}(27 \mathrm{in})$ in $\mathrm{Y}$ and $1.5 \mathrm{~m}(60 \mathrm{in})$ in $\mathrm{Z}$. Significant space is required above the plane of the $\mathrm{X}-\mathrm{Y}$ axis to accommodate the $\mathrm{Z}$ tower, thus the requirement for "double-decker" gloveboxes. Two television cameras were mounted on the robot for tele-operation. The containers handled were either 55-gallon drums or DOT 7A containers, which are made of steel and have dimensions of $2.18 \mathrm{~m}$ (86 in) long x $1.17 \mathrm{~m} \mathrm{(46} \mathrm{m)}$ wide x $1.07 \mathrm{~m}$ (42 in) deep. Both autonomous and tele-operated control methods were specified. According to [Dennison, et al, 1995], an IBM Series 2 controller controlled the system. The literature review revealed that this system does not seem ideally suited to the needs of Plutonium processing for several reasons: (1) The volume and payload are larger than necessary, thus requiring larger and more expensive gloveboxes, (2) Linear actuators contain much more sliding contact area and open components which are susceptible to wear from abrasive Plutonium particles, and (3) The system must be constructed and repaired inside a glovebox and the large linear components cannot be bagged out. As a general observation, the three axis Cartesian configuration does not seem to be a good choice for any "hot" glovebox operation given the volume required to clear the Z-axis tower.

In approximately March of 1996, the U.S. subsidiary of British Nuclear Fuels, BNFL Inc., was awarded a contract to supply technology for a PuSPS at the Rocky Flats Environmental 
Test Site (RFETS) [BNFL, 1996]. The RFETS has the requirement to stabilize and package more than $6,600 \mathrm{~kg}\left(14,553 \mathrm{lb}\right.$ ) of Plutonium metal and 3,200 $\mathrm{kg}\left(7056 \mathrm{lb}_{\mathrm{m}}\right)$ of plutonium compounds. ([Rocky Flats, 1998], pg. 7). The packaged materials will then primarily be transferred to the WIPP in New Mexico for storage and SRS waste processing. The BNFL system combined a furnace for heat-drying Plutonium oxides and a packaging system to place the processed material into 50-year storage containers. This highly automated system places most of the equipment inside the glovebox. The system was delivered in September 1998 to Kaiser-Hill [Roberson, 1998]. The original system specifications called for a throughput of two (2) cans every eight (8) hours. However, the system is still in a qualification stage due to a problem with radioactive contamination around the seal of the weld and is expected to get deployed sometime in DOE's FY2001 [RFCAB, 2000]. At present, no automation regarding material transfer has been implemented in this system even though it was originally planned [BNFL, 1998].

There are two acceptable methods per the START agreements for disposing of the excess weapons grade plutonium: 1) convert the plutonium into a Metal Oxide (MOX) fuel and burn it in a reactor, or 2) immobilize the plutonium and store it in a facility where it can be monitored while it radiologically decays to a stable material [DOE, 2000a]. LANL is developing a system that utilizes the first approach for plutonium disposition called the Advanced Recovery and Integrated Extraction System (ARIES). SRS is following the second method under a project called the Plutonium Immobilization Project (PIP). From a material handling point of view, both systems have equal requirements since each system utilizes the DOE standard 3013 canisters for packaging the plutonium.

The SRS PIP will process high-grade plutonium and plutonium compounds into ceramic pucks and seal the pucks inside 3013 canisters. The process will consist of three stages: Plutonium Conversion, First Stage Immobilization, and Second Stage Immobilization [Dyches, et al, 1999]. The first stage pertains to the following operations: material receipt, unpacking and characterization, conversion of its MOX forms, and material size reduction. In the second stage, the oxide metal is immobilized into a ceramic form. During this stage, the material is formed into pucks weighing approximately one pound. The last stage involves the packaging of the pucks into the 3013 canisters and then into Defense Waste Processing Facility (DWPF) canisters where they can be transferred to a storage facility. Figure 1 shows a conceptual drawing of the proposed puck handling and can loading system [Dyches, et al, 1999]. This system is designed to utilize two robotic systems: an overhead gantry for handling the pucks and a 6 DOF system for moving the 3013 canister from station to station. To date, only the puck handling manipulator system has been developed and tested ([Kriikku, et al, 1998] and [Kriikku, et al, 1999]). The puck robot is a 3 DOF system with a vacuum cup for a gripper. The travel along the three Cartesian axes is specified as follows: $\mathrm{X}-1.32 \mathrm{~m}(52$ "), $\mathrm{Y}-1.02 \mathrm{~m}$ (40"), and Z $1.02 \mathrm{~m}$ (40"). Its maximum target payload is one full 3013 canister weighing approximately 13.6 $\mathrm{kg}(30 \mathrm{lbs})$. Each axis is specified to have a repeatability of $0.25 \mathrm{~mm}(0.010$ inches $)$ and a velocity range of $0-0.2 \mathrm{~m} / \mathrm{sec}(0-8 \mathrm{in} / \mathrm{sec})$ [Kriikku, et al, 1998]. No accuracy numbers were given. The can robot is specified to be a 6 DOF system with a maximum payload of $11.4 \mathrm{~kg}(25$ $\mathrm{lbs})$. The required maximum radial reach is $0.76 \mathrm{~m} \mathrm{(30").} \mathrm{The} \mathrm{repeatability} \mathrm{and} \mathrm{velocity}$ requirements are $0.5 \mathrm{~mm}\left(0.020^{\prime \prime}\right)$ and $0-0.7 \mathrm{rad} / \mathrm{sec}(0-40 \mathrm{deg} / \mathrm{sec})$, respectively. The gripper

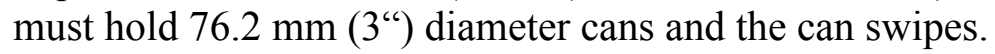




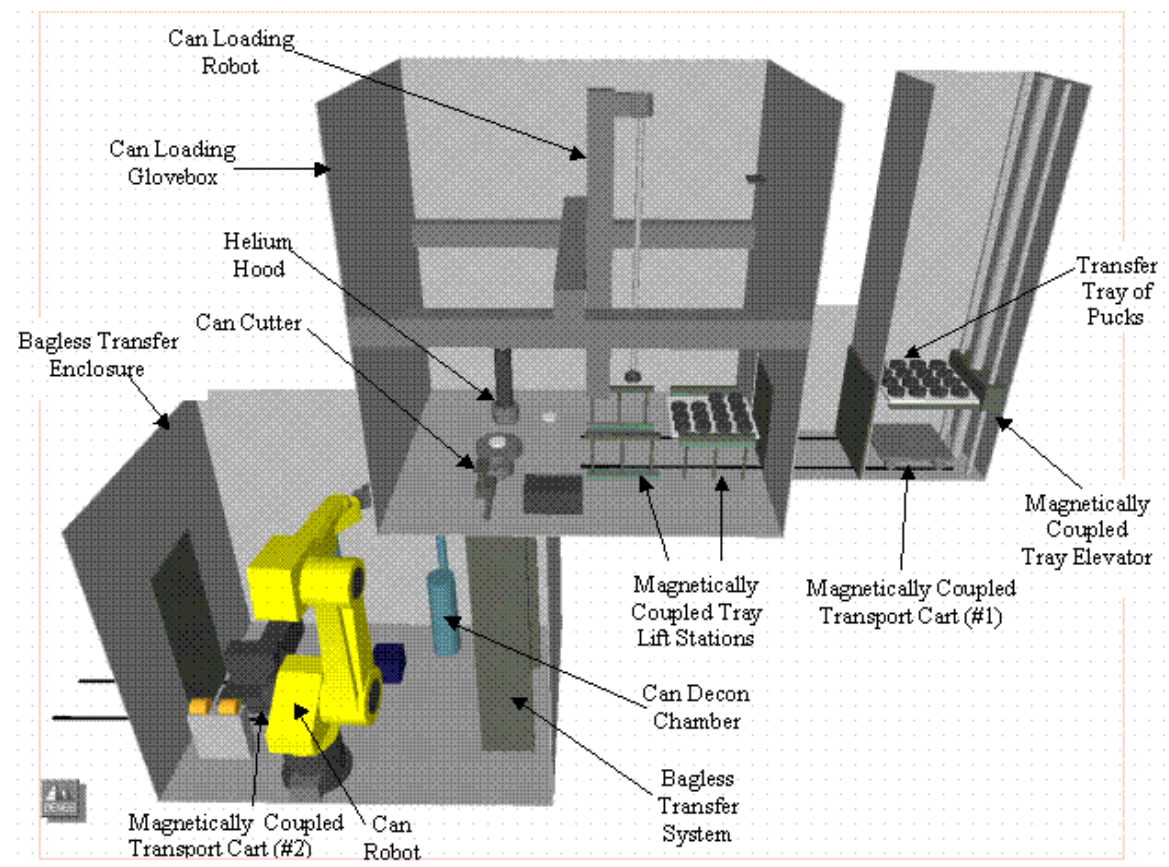

Figure 1: SRS PIP's stage 3 material handling system's conceptual design.

As currently designed, ARIES contains a mix of manual and automated material handling operations. As technology and time permit, automation of additional processes may be considered. The ARIES system consists of several gloveboxes, which are interconnected via a conveyor system. A different process is performed within each glovebox. The processes are:

1. Pit Bisector

2. Hydride-Dehydride Furnace

3. Hydrox Furnace

4. Canning

5. Electro-decontamination

To ensure tight control over the amount of Plutonium processed, it is weighed both upon entering and exiting each glovebox. A final step, Non-Destructive Assay (NDA), is performed outside of the gloveboxes. The output of the system is a decontaminated, sealed 3013 container, containing a known quantity of Plutonium metal or oxide. When in production, the containers will ultimately proceed to either long-term storage or a MOX fuel production facility.

Sandia National Laboratories (SNL) is designing an automated cell for the ARIES line. In this glovebox, SNL is combining two operations: welding and electro-decontamination of the material container. The system utilizes two FANUC LR-Mate 100i robots, one on the 'hot' side and one on the cold side (see Figure 2). The LR-Mate 100i's specifications are shown in Table 2. This robot is traditionally used for part loading operations. While not ideal for the task at hand, it is the best commercially available option. The biggest problem with this manipulator is maintenance and repair within the glovebox. Even a task as simple as removing the cap from the grease zerk fitting becomes nearly impossible with gloves. Consequently, very few failures will be repairable inside the glovebox, and hence, the entire LR-Mate is viewed as a disposable unit. This creates significant challenges in several areas: 1) Removal and replacement of the manipulator, 2) Disposal of the failed manipulator and 3) Calibration of the new manipulator. After significant effort, the SNL team has devised a clever system by which a failed LR-Mate 

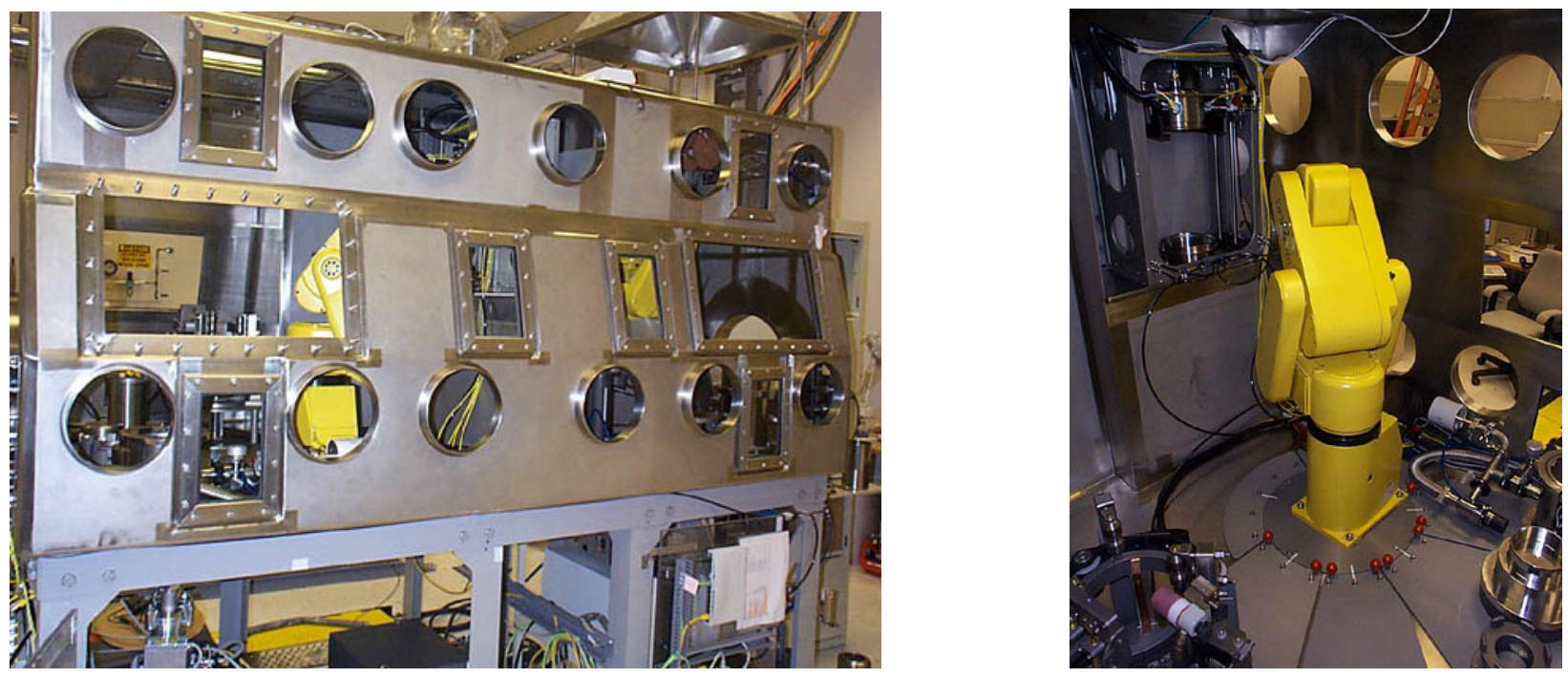

Figure 2: Two LR-Mates operational in an ARIES glovebox (left). LR-Mate viewed from "hot" side of glovebox (right).

may be lowered down through a large portal in the glovebox into a bag and drummed for disposal. This system utilizes a linear guideway system to prevent Plutonium spillage due to the robot tipping over. The resulting operation is elaborate and requires time and care to perform. Despite the novel approach, it still has the additional disadvantage in that it cannot be retrofitted to existing gloveboxes and has yet to be qualified for safety [McKee, 1998].

\subsubsection{Glovebox Manipulator And Actuator Requirements}

Following the completion of the topical report presented in [Black, 1998], ARM proposed to develop under this project two manipulator designs for automating glovebox operations: (1) A flexible, more complex system which meets the needs of a wide range of

\begin{tabular}{|c|c|c|c|c|c|}
\hline Item & \multicolumn{5}{|c|}{ Specification } \\
\hline Degrees of freedom & \multicolumn{5}{|l|}{5} \\
\hline Payload & \multicolumn{5}{|c|}{$3 \mathrm{~kg}\left(6.6 \mathrm{Ib}_{\mathrm{m}}\right)$} \\
\hline $\begin{array}{l}\text { Payload (reduced } \\
\text { speed) }\end{array}$ & \multicolumn{5}{|c|}{$4 \mathrm{~kg}(8.8 \mathrm{lb})$} \\
\hline Mass & \multicolumn{5}{|c|}{$32 \mathrm{~kg}\left(70.5 \mathrm{lb}_{\mathrm{m}}\right)$} \\
\hline Repeatability & \multicolumn{5}{|c|}{$+/-0.04 \mathrm{~mm}(+/-0.002 \mathrm{in})$} \\
\hline $\begin{array}{l}\text { End Effector } \\
\text { Pneumatics }\end{array}$ & \multicolumn{5}{|c|}{4 standard, 6 optional } \\
\hline Reach & \multicolumn{5}{|c|}{$620 \mathrm{~mm}(24.4 \mathrm{in})$} \\
\hline Axis Specifications & $\begin{array}{l}\text { Motion } \\
\text { Range }\end{array}$ & $\begin{array}{l}\text { Speed } \\
\text { (\%sec) }\end{array}$ & $\begin{array}{l}\text { Moment } \\
(\mathrm{N} \cdot \mathrm{m})\end{array}$ & $\begin{array}{l}\text { Inertia } \\
\left(\mathrm{kg} \cdot \mathrm{cm} \cdot \mathrm{s}^{2}\right)\end{array}$ & Brake \\
\hline 1 & $300^{\circ}$ & 150 & - & & Optional \\
\hline 2 & $180^{\circ}$ & 150 & - & & $\mathrm{Y}$ \\
\hline 3 & $135^{\circ}$ & 180 & - & & $\bar{Y}$ \\
\hline $\begin{array}{l}4 \\
\text { (reduced speed) }\end{array}$ & $240^{\circ}$ & $\begin{array}{c}170 \\
(100)\end{array}$ & $\begin{array}{c}5.39 \\
(6.86) \\
\end{array}$ & $\begin{array}{l}1.05 \\
(1.4)\end{array}$ & $\bar{N}$ \\
\hline 5 & $360^{\circ}$ & 240 & 3.92 & .41 & $\mathrm{~N}$ \\
\hline
\end{tabular}

Table 2: FANUC LR-Mate 100i specifications [FANUC, 1993]. 


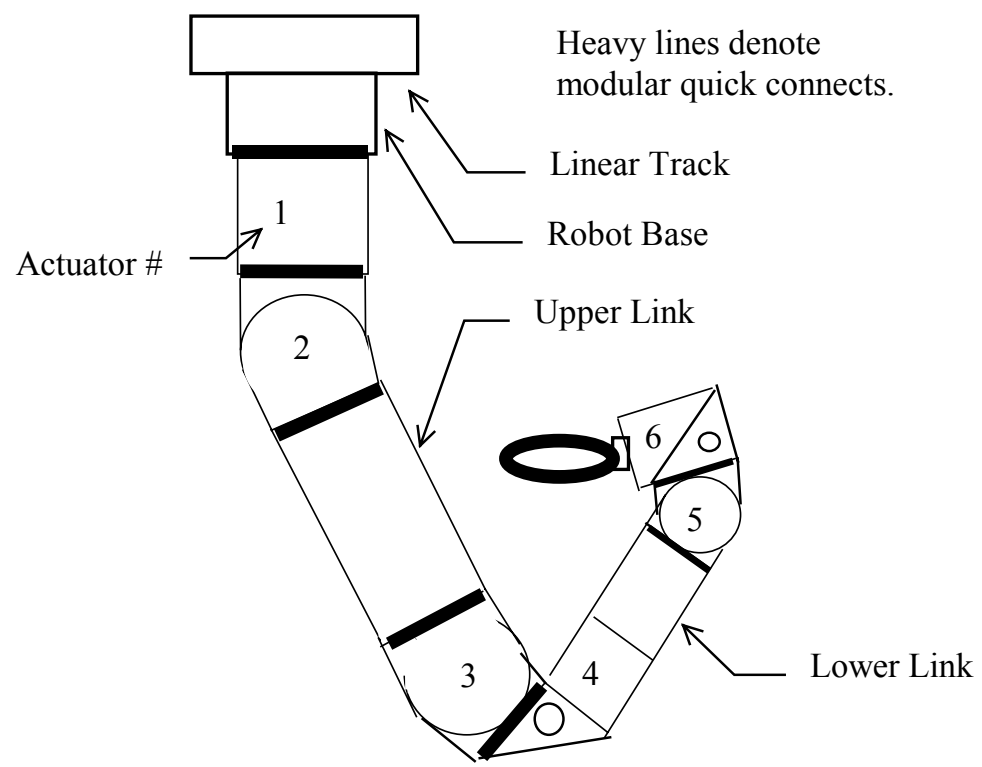

Figure 3: 6-DOF manipulator suspended from track.

glovebox tasks and (2) One less complicated, which meets the specific requirements of the ARIES or PIP automation cells. The beauty of the modular approach shines as the second system is simply constructed by removing modular components from the first, and can be configured within a brief amount of time. The first system was proposed to be built and demonstrated at ARM's facility while the second system was to be tested at a designated DOE site. This strategy tests the ability of the modular approach to accommodate multiple system reconfigurations. The following describes the requirements envisioned for each system. Both systems were to be controlled with the same open-architecture PC-based system controller.

Based upon needs presented in [Black, 1998], ARM proposed the following for the versatile Plutonium glovebox automation system: A 5-DOF Pitch-Pitch-Roll-Pitch-Roll (PPRPR) manipulator attached to a linear track with the axis of the first pitch actuator parallel to the axis of the linear track. It was originally proposed to suspend the linear track as shown in Figure 3 but the same tests were carried out with the same manipulator configuration mounted flat on the table. The resulting system was to be controlled as a six (6) DOF manipulator. This approach simplified tele-operation by eliminating the control issues associated with redundant (systems containing more than six (6) DOF) manipulators. Future systems may use a more traditional (6) DOF manipulator (the above PPRPR with a first Roll joint added) on the linear track. The target reach was to be $1.02 \mathrm{~m}(40$ ") with a $10 \mathrm{~kg}(22 \mathrm{lb}$ ) payload. The linear track will have a travel of approximately $1 \mathrm{~m}$ (39.4 in). UT simulated the most complex system anticipated for glovebox use to ensure the appropriate sizing of the actuators.

The requirements of the DOE site demonstration were among the least demanding of any of the systems evaluated for [Black, 1998] and summarized in Table 1. The end-effector was allowed to be either pneumatic or electric within a range of voltages. Only on/off control of the voltage or pneumatic supply was deemed necessary. Ideally, the payload was to be $10 \mathrm{~kg}(22$ $\left.\mathrm{lb}_{\mathrm{m}}\right)$ with an absolute minimum of $5 \mathrm{~kg}(11 \mathrm{lb})$. To prevent wear due to Plutonium particles, as many of the joints as possible were to be sealed and positively pressurized. Discussions at the kick-off presentation lead to the conclusion that four (4) DOF were sufficient for this task. 


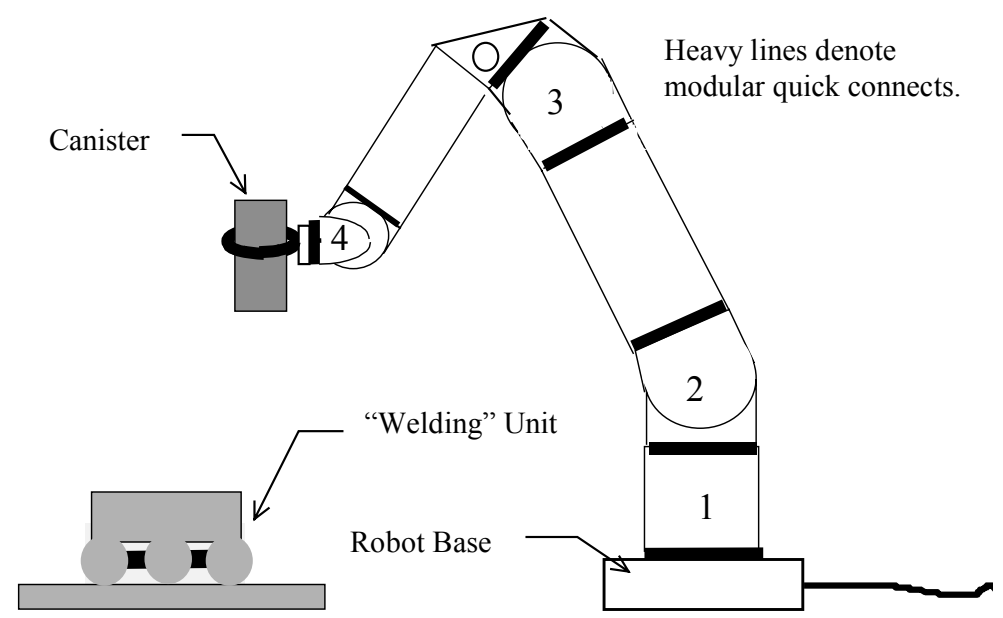

Figure 4: 4-DOF robot for upright canister transfer.

Hence, an RPPP configuration was proposed for this application, which is a subset of the PPRPR configuration (described above) with the last two roll joints removed and a first roll joint added (see Figure 4). The most unique requirement of this system stems from the fact that the system is teach-pendant taught and cannot be easily re-taught once installed in the glovebox. As a result, the manipulator must be repeatable after module replacement or manipulator re-assembly. This mode of operation may require a generic manipulator calibration scheme but that was deemed to be beyond the original scope of the project.

Due to the versatility of the modular approach, requirements of the other DOE tasks forced little compromise in the specifications for the APP manipulator design, but careful thought was given to the actuator level decisions. There were two classes of decisions that were made with regard to the actuator designs introduced. The first decision determined which actuator sizes from the available spectrum were appropriate for this manipulator system. The second class of decisions determined actuator level performance and design specifications.

With regard to the actuator spectrum, it was not desirable to design both modules with approximately equal capacities even if that was sufficient for APP. To ensure a maximum of versatility with the initial two designs, they were spaced as to maximize their usability in other applications without being severely oversized for the APP task. These architectural decisions were made as the modules reached final design and the APP actuator torque requirements were determined through simulation.

Some of the more significant decisions that were made during the kick-off meeting are now described. For aesthetic and mechanical reasons, a decision was made to pass the wire internally through the links and actuators with connectors integrated into a single electromechanical interface. While difficult to achieve, this approach results in a clean, robust design that can be quickly and easily reconfigured. Feasibility demands that the number and size of wires be kept to a minimum, due to space requirements. In addition to volume, the number of wires also has a critical interrelationship on the degrees of rotation required. While not required for APP, a decision was made to include pass through power for operation of end-effectors, such as in D\&D applications. The intent to pass-through pneumatics was to be included during the design process of the actuators and links.

The last major architectural decision stemming from the kick-off meeting involved jointlevel torque sensing. Some robotics purists prefer to resolve end-effector force/torque load 

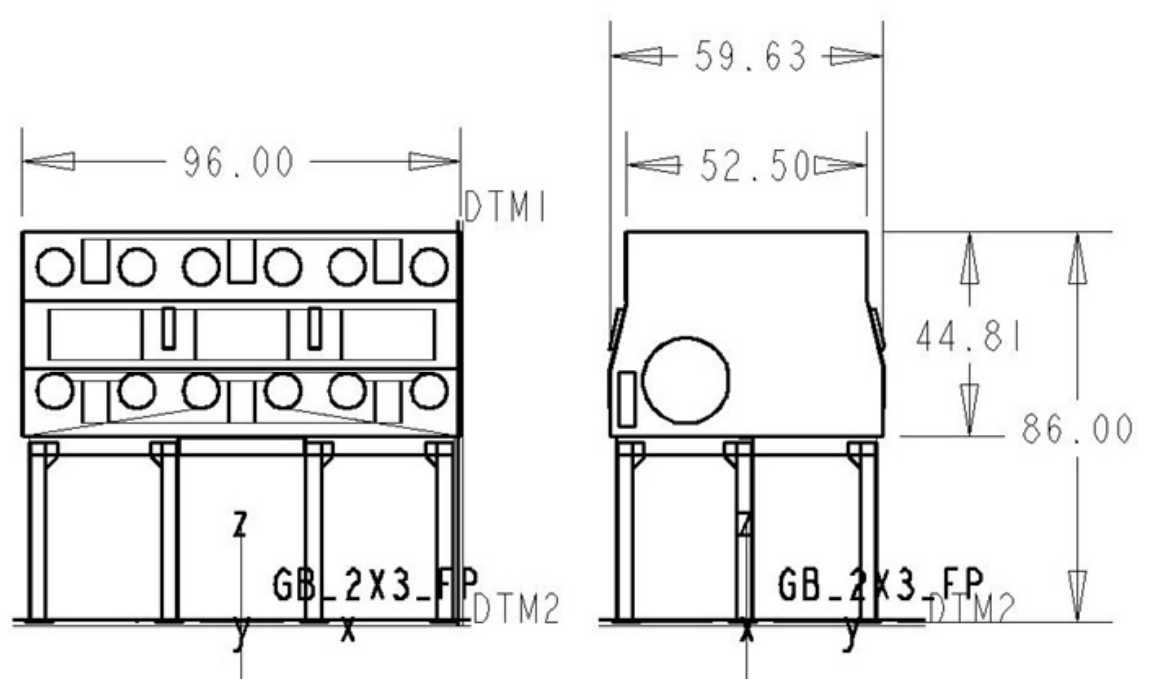

Figure 5: Dimensions of simulated APP glovebox.

measurement based upon joint level torque measurements for force-controlled tele-operated systems. Joint torque sensing allows a system to operate in a more advanced torque mode. At the kick-off meeting, it was not believed that feature was worth the cost and complexity for the scope of this project, and thus, was not incorporated in the actuator designs. Other requirements, such as positional accuracy and sensor resolution, were derived as designs matured to insure a maximum of versatility across all actuator applications. It was only important that these specifications exceeded those of the LR-Mate $100 i$ presented in the previous section.

A final requirement arose during the kick-off meeting. The designs envisioned to date incorporate a finned housing to facilitate heat-transfer. It was observed that any non-smooth surface, such as fins, complicates decontamination and should be avoided if possible. A final decision will be dependent upon heat transfer and load calculations, but smooth surfaces were to be incorporated wherever possible.

\section{RESULTS AND DISCCUSSION}

\subsection{Glovebox Manipulator Simulation}

To aid in the design of this manipulator and its actuators, ARM subcontracted the UT Robotics Research Group (UTRRG) to simulate the manipulator in its actual working environment. However, instead of simulating a 4 or 5 degree of freedom manipulator, ARM requested that the UTRRG simulate a 6 DOF robot mounted on a positioning gantry in the roof of the glovebox. The simulation was designed to feature a more elaborate system as a means of demonstrating the application of a more advanced manipulator.

To accomplish this task, the UTRRG was to create a three-dimensional model of the proposed manipulator. UT then used this model to graphically simulate the proposed manipulator to test its capabilities and aid in setting the design specifications for torque (peak and continuous) and speed. The simulation specifications are shown in Table 3. Figure 5 depicts the dimensions of the glovebox used in the simulation. The UTRRG's plan to complete this objective consisted of a five step approach including:

1. Creating the three-dimensional graphical model of the proposed manipulator based upon delivered specifications. 


\begin{tabular}{|c|c|c|c|c|c|c|}
\hline Category & \multicolumn{6}{|c|}{ Manipulator Specifications } \\
\hline \multirow[t]{6}{*}{ Functional Requirements: } & \multicolumn{6}{|c|}{ End Effector Payload must be at least $10 \mathrm{~kg}$ at 6 inches from tool plate } \\
\hline & \multicolumn{6}{|l|}{6 DOF Manipulator } \\
\hline & \multicolumn{6}{|l|}{ Positioning Linear Track } \\
\hline & \multicolumn{6}{|c|}{ Actuators $1,2,3$ are large actuators } \\
\hline & \multicolumn{6}{|c|}{ Actuators $4,5,6$ are small actuators } \\
\hline & \multicolumn{6}{|c|}{ Manipulator Configuration R-P-P-R-P-R } \\
\hline \multirow[t]{2}{*}{ Materials: } & \multicolumn{6}{|c|}{ Anodized Aluminum Actuators and Yokes } \\
\hline & \multicolumn{6}{|c|}{ Stainless Steel Modular Connectors } \\
\hline \multirow[t]{8}{*}{ Geometry: } & \multicolumn{6}{|c|}{12 inch distance between axis of actuator 2 and ceiling of glovebox } \\
\hline & \multicolumn{6}{|c|}{20 inch distance between axis of actuator 3 and actuator 2} \\
\hline & \multicolumn{6}{|c|}{5.93 inch distance offset between axis of actuator 4 and actuator 3} \\
\hline & \multicolumn{6}{|c|}{18 inch distance between projection axis of actuator 3 and actuator 5} \\
\hline & \multicolumn{6}{|c|}{5.09 inch distance offset between axis of actuator 6 and actuator 5} \\
\hline & \multicolumn{6}{|c|}{2.56 inch distance between bottom face of actuator 4 and projected axis of actuator 3} \\
\hline & \multicolumn{6}{|c|}{ Bottom face of actuator 4 is tangent to radius of actuator 3 (large actuator) } \\
\hline & \multicolumn{6}{|l|}{ Tool Geometry } \\
\hline \multirow[t]{3}{*}{ Kinematics: } & \multicolumn{6}{|c|}{ Maximum velocity of $200 \mathrm{~mm} / \mathrm{sec}$} \\
\hline & \multicolumn{6}{|c|}{ Maximum Acceleration of $0.5 \mathrm{~g}$ in vertical } \\
\hline & \multicolumn{6}{|c|}{ Maximum Acceleration of $2 \mathrm{~g}$ in transverse } \\
\hline Inertial Parameters: & Upper link $\mathrm{m}=2 \mathrm{~kg}$ & & & & & \\
\hline & Lower link $\mathrm{m}=1.25 \mathrm{~kg}$ & & & & & \\
\hline & Larger Half Yoke $\mathrm{m}=1$ & & & & & \\
\hline & Small Half Yoke $m=0$. & & & & & \\
\hline & Small Actuator $\mathrm{m}=2.5$ & & & & & \\
\hline & Large Actuator $\mathrm{m}=6 \mathrm{k}$ & & & & & \\
\hline & Tool Mass $=1.25 \mathrm{~kg}$ & & & & & \\
\hline DH Parameters: & Actuator & Joint & $a_{i-1}$ & $d_{i}$ & $\alpha_{i-1}$ & $\theta_{\mathrm{i}}$ \\
\hline & 0 & 1 & $0 "$ & Var. & $90^{\circ}$ & $0^{\circ}$ \\
\hline & 1 & 2 & $0 "$ & $8 ”$ & $90^{\circ}$ & Var. \\
\hline & 2 & 3 & $20 "$ & $0 "$ & $0^{\circ}$ & Var. \\
\hline & 3 & 4 & $5.93 ”$ & $0 ”$ & $-90^{\circ}$ & Var. \\
\hline & 4 & 5 & $0 "$ & $18 ”$ & $-90^{\circ}$ & Var. \\
\hline & 5 & 6 & $5.09 ”$ & $0 "$ & $-90^{\circ}$ & Var. \\
\hline & 6 & 7 & $0 "$ & tool & $0^{\circ}$ & Var. \\
\hline & Prismatic joint is assum & not cont & to overa & & & \\
\hline Actuator Dimensions: & Large Actuator & & & & & \\
\hline & Diameter $=0.130 \mathrm{~m}$ & & & & & \\
\hline & Length $=0.150 \mathrm{~m}$ & & & & & \\
\hline & Small Actuator & & & & & \\
\hline & Diameter $=0.095 \mathrm{~m}$ & & & & & \\
\hline & Length $=0.105 \mathrm{~m}$ & & & & & \\
\hline Environmental: & Wall thickness 0.1 inch & & & & & \\
\hline & Obstacles modeled as cy & rs and $b$ & ss than & hes tall & & \\
\hline
\end{tabular}

Table 3: UTRRG APP glovebox manipulator parameters.

2. Developing inertial parameters and composite centroids for the manipulator.

3. Developing an aggressive path plan.

4. Analyzing actuator torques using constant speed path.

5. Analyzing actuator torques using a motion program over the path.

From the completion of these steps, the resulting continuous torque values guided ARM's actuator design process. 


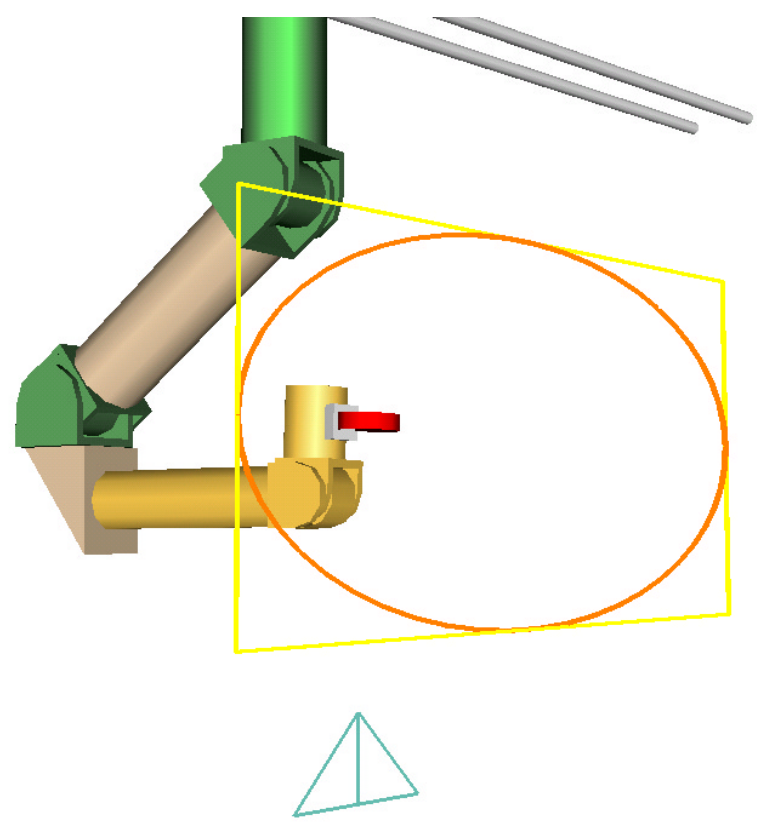

Figure 6: Graphic simulation model. The triangle was placed nearly in line with actuator one's primary axis.

A snap shot of the graphic simulation of the model shown in Figure 6 was based upon a preliminary geometry provided by ARM. While this was not the final geometry used, it represented a good first approximation for guiding future design decisions. The outlines of the rectangle, circle, and triangle represent paths that the manipulator traced with the end-effector to complete the path. The path was originally designed to aggressively test the manipulator very near to its singularities. While the paths did accomplish this objective, later analysis proved to be difficult due to the very high joint torques induced as the manipulator quickly moved around singularities. Similarly, the simulation produced very high torques tracing the hard corners of the rectangle and the triangle. To combat this difficulty in the simulation's results, UT performed the simulation with a linear deceleration as the end effector approached corners. For comparison, the simulation ran over several velocities including: 1, 5, 10, and 20 inches per second. For further comparison, the same simulations were run for the same maximum velocities using a cosine motion profile program. The resulting simulation still showed very high torques when operating near singularities. For the final analysis, the maximum torque of the joint actuators was limited to a practical value. This produced a much more useful set of data. The final results of the constant velocity and cosine motion profile simulations for 10 and $20 \mathrm{in} / \mathrm{sec}$ tip speed are presented in Table 4.

The results of the UT simulation combined with those acquired from the topical report led to the target design specifications summarized in Table 5. The ARM20 torque specifications exceed those determined by the UT simulation for Actuators 4-6. The ARM32 torque specifications meet those determined by the UT simulation for Actuators 1 and 3 for all paths and for Actuator 2 for all paths except $20 \mathrm{in} / \mathrm{sec}$ with constant velocity. The peak ratings for Actuator 2 do exceed those given in Table 5 .

Based on the above specifications for the actuators, the proposed manipulator ARM is to deliver to a DOE site for the glovebox operations will have the specifications shown in Table 6. 


\begin{tabular}{|c|c|c|c|c|c|c|}
\hline Actuator 1 & Fx & Fy & $\mathbf{F z}$ & Tx & Ty & Tz \\
\hline 10 Inches per second with constant velocity & 76.67 & 46.29 & 520.10 & 22.56 & 123.81 & 22.21 \\
\hline 20 Inches per second with constant velocity & 306.60 & 184.57 & 526.45 & 80.97 & 198.59 & 88.76 \\
\hline 10 Inches per second with Cosine Motion Program & 29.89 & 24.12 & 519.60 & 16.77 & 123.00 & 14.59 \\
\hline 20 Inches per second with Cosine Motion Program & 93.50 & 94.80 & 520.36 & 64.38 & 141.26 & 65.50 \\
\hline Actuator 2 & $\mathbf{F x}$ & Fy & $\mathbf{F z}$ & Tx & Ty & Tz \\
\hline 10 Inches per second with constant velocity & 406.65 & 151.23 & 46.29 & 11.30 & 28.00 & 123.81 \\
\hline 20 Inches per second with constant velocity & 406.07 & 343.69 & 184.57 & 44.41 & 104.91 & 198.59 \\
\hline 10 Inches per second with Cosine Motion Program & 404.92 & 138.90 & 24.12 & 11.31 & 20.57 & 123.00 \\
\hline 20 Inches per second with Cosine Motion Program & 400.89 & 172.36 & 94.80 & 50.63 & 70.89 & 141.26 \\
\hline Actuator 3 & $\mathbf{F x}$ & Fy & $\mathbf{F z}$ & Tx & Ty & Tz \\
\hline 10 Inches per second with constant velocity & 258.90 & 197.68 & 39.17 & 7.47 & 9.92 & 97.50 \\
\hline 20 Inches per second with constant velocity & 322.52 & 265.75 & 156.05 & 23.02 & 28.21 & 100.24 \\
\hline 10 Inches per second with Cosine Motion Program & 252.72 & 195.88 & 20.05 & 5.28 & 10.41 & 96.24 \\
\hline 20 Inches per second with Cosine Motion Program & 257.93 & 213.15 & 87.99 & 24.89 & 31.06 & 99.71 \\
\hline Actuator 4 & $\mathbf{F x}$ & Fy & $\mathbf{F z}$ & Tx & Ty & Tz \\
\hline 10 Inches per second with constant velocity & 184.30 & 15.00 & 141.08 & 5.88 & 5.13 & 8.14 \\
\hline 20 Inches per second with constant velocity & 193.39 & 59.23 & 167.42 & 13.07 & 15.05 & 18.71 \\
\hline 10 Inches per second with Cosine Motion Program & 181.50 & 8.49 & 142.33 & 5.67 & 3.74 & 8.83 \\
\hline 20 Inches per second with Cosine Motion Program & 182.61 & 43.54 & 149.94 & 14.10 & 21.33 & 29.45 \\
\hline Actuator 5 & $\mathbf{F x}$ & Fy & Fz & Tx & Ty & Tz \\
\hline 10 Inches per second with constant velocity & 12.55 & 186.59 & 9.90 & 8.65 & 0.24 & 10.92 \\
\hline 20 Inches per second with constant velocity & 49.87 & 190.58 & 39.00 & 9.14 & 0.98 & 11.44 \\
\hline 10 Inches per second with Cosine Motion Program & 3.05 & 186.35 & 3.06 & 9.16 & 1.08 & 10.58 \\
\hline 20 Inches per second with Cosine Motion Program & 23.73 & 188.10 & 29.42 & 11.61 & 4.31 & 21.13 \\
\hline Actuator 6 & Fx & Fy & Fz & Tx & Ty & Tz \\
\hline 10 Inches per second with constant velocity & 3.75 & 7.70 & 154.55 & 1.03 & 13.89 & 0.22 \\
\hline 20 Inches per second with constant velocity & 14.47 & 30.68 & 155.96 & 4.12 & 14.06 & 0.88 \\
\hline 10 Inches per second with Cosine Motion Program & 2.02 & 1.66 & 154.52 & 0.40 & 13.90 & 1.03 \\
\hline 20 Inches per second with Cosine Motion Program & 16.26 & 23.84 & 156.06 & 19.35 & 14.20 & 4.12 \\
\hline
\end{tabular}

Table 4: Simulation RMS values (values given in $\mathrm{N}$ for force and $\mathrm{N} \cdot \mathrm{m}$ for torque).

Figure 7 shows the resulting four-axis configuration based on these specifications. It should be noted that these specifications exceed those given for the LR-Mate in Table 2.

\begin{tabular}{|l|c|c|}
\hline & ARM20 & ARM32 \\
\hline Performance: & & \\
\hline • Range of Motion & $\pm 340^{\circ}($ or continuous*) & $\pm 340^{\circ}($ or continuous*) \\
\hline • Peak Speed & $30 \mathrm{RPM}$ & $35 \mathrm{RPM}$ \\
\hline - Peak Torque & $82 \mathrm{~N} \cdot \mathrm{m}(60 \mathrm{ft} \cdot \mathrm{lb})$ & $333 \mathrm{~N} \cdot \mathrm{m}(245 \mathrm{ft} \cdot \mathrm{lb})$ \\
\hline - Continuous Torque & $40 \mathrm{~N} \cdot \mathrm{m}(30 \mathrm{ft} \cdot \mathrm{lb})$ & $137 \mathrm{~N} \cdot \mathrm{m}(101 \mathrm{ft} \cdot \mathrm{lb})$ \\
\hline • Bearing Load Capacity & $3330 \mathrm{~N}(740 \mathrm{lb})$ & $7550 \mathrm{~N}(1716 \mathrm{lb})$ \\
\hline • Bearing Overturning Load & $333 \mathrm{~N} \cdot \mathrm{m}(245 \mathrm{ft} \cdot \mathrm{lb})$ & $755 \mathrm{~N} \cdot \mathrm{m}(555 \mathrm{ft} \cdot \mathrm{lb})$ \\
\hline Physical: & & $160 \mathrm{~mm}(6.3 \mathrm{in})$ \\
\hline - Length & $128 \mathrm{~mm}(5.0 \mathrm{in})$ & $145 \mathrm{~mm}(5.7 \mathrm{in})$ \\
\hline - Diameter & $97 \mathrm{~mm}(3.8 \mathrm{in})$ & $4.5 \mathrm{~kg}(9.9 \mathrm{lb}$ m $)$ \\
\hline - Mass & $2.3 \mathrm{~kg}\left(5 \mathrm{lb} \mathrm{b}_{\mathrm{m}}\right)$ & $100: 1$ \\
\hline - Gear Train Ratio & $100: 1$ & \\
\hline
\end{tabular}




\begin{tabular}{|c|c|}
\hline Parameter & Preliminary Specification \\
\hline \hline Degrees of freedom & 4 \\
\hline Payload & $12 \mathrm{~kg}$ \\
\hline Maximum reach & $750 \mathrm{~mm}$ \\
\hline Peak end effector speed & $1.5 \mathrm{~m} / \mathrm{s}$ \\
\hline Weight (including cable) & $28 \mathrm{~kg}$ \\
\hline \# of modules & 4 \\
\hline Module Types and quantity of each & 1 ARM20 \& 3 ARM32 \\
\hline System controller & Cimetrix controller running on an NT platform \\
\hline End effector & Modified electric Sandia constricting band \\
\hline Mechanical Interfaces & ARM proprietary quick connects \\
\hline
\end{tabular}

Table 6: Preliminary 4 DOF glovebox manipulator specifications.

\subsection{Manipulator Component Development}

The manipulator components designed and fabricated included two sizes of actuator modules; two sizes of links, quick-connects, and yokes; umbilical cord; power supply, and system controller. UT performed the testing of the actuators under this task to verify the design parameters. The remaining sub-sections will expound on the development process of these manipulator components.

\subsubsection{Actuator Development}

For this project, two sizes of modular actuators were developed; an ARM20 and an ARM32. These actuators were designed to meet the specifications set forth in the previous sections. The design of the actuators involves the development and fabrication of the $\mathrm{DISC}^{\mathrm{TM}} \mathrm{S}$, and the input and output sections for each actuator. The DISC ${ }^{\mathrm{TM}}$ development included the design of a Controller and Communications Interface (CCI) board common to each system and a Power and Sensor Interface (PSI) board sized to each actuator's power and sensor requirements.

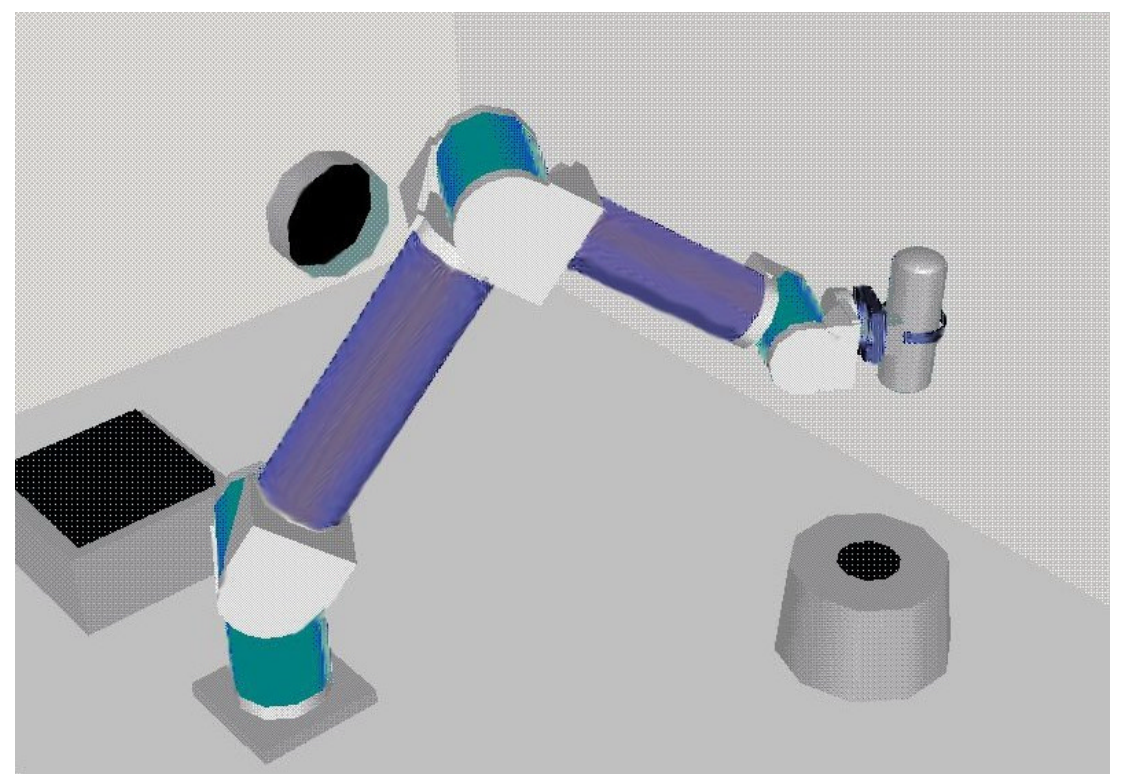

Figure 7: Model of 4 DOF glovebox manipulator. 


\begin{tabular}{|l|c|c|}
\hline \multicolumn{1}{|c|}{ Parameter } & Value & Units \\
\hline \hline Rotor Inertia & $6.64 \times 10^{-5}$ & $\mathrm{~kg} \cdot \mathrm{m}^{2}$ \\
\hline Temperature Rise Per Watt & 2.6 & \\
\hline Detent Torque & 0.0177 & $\mathrm{~N} \cdot \mathrm{m}$ \\
\hline Torque Constant & 0.632 & $\mathrm{~N} \cdot \mathrm{m} / \mathrm{A}$ \\
\hline Voltage Constant & 0.632 & $\mathrm{~V} / \mathrm{rad} / \mathrm{sec}$ \\
\hline Winding Resistance & 18.3 & $\Omega$ \\
\hline Terminal Inductance & 22.6 & $\mathrm{mH}$ \\
\hline Continuous Rated Torque & 0.9 & $\mathrm{~N} \cdot \mathrm{m}$ \\
\hline No Load Speed & 367 & $\mathrm{rad} / \mathrm{sec}$ \\
\hline Peak Torque & 8.31 & $\mathrm{~N} \cdot \mathrm{m}$ \\
\hline Peak Current & 13.2 & $\mathrm{~A}$ \\
\hline Designed Voltage & 230 & $\mathrm{Volts}$ \\
\hline Number of Poles & 12 & Poles \\
\hline Hall (A-B-C) Configuration & $0 \times 321$ & - \\
\hline Winding Configuration & Delta & - \\
\hline
\end{tabular}

Table 7: ARM20 motor specifications.

Following the complete fabrication and testing of these individual components, each of the modules were assembled and fully tested by ARM and UT.

\subsubsection{ARM20 Development}

The ARM20 development encompassed the development of the ARM20 mechanicals, the CCI, and the PSI20. While the CCI board is common to both the ARM20 and ARM32 modules, its development took place under the ARM20 development. The sub-sections below describe the development process for the ARM20. The actuator testing of the ARM20 is included with that of the ARM32 in Section 2.2.1.4. When readily available, ARM obtained the information regarding the radiation resistance of specific components and selected the radiation resistant components when they were not cost prohibitive. Many manufacturers did not have data regarding the radiation resistance of their components and advised ARM to thoroughly test the devices in a system in order to fully determine the radiation hardness of the system. Since there were many technological developments under this contract, ARM chose to delay the qualification of the radiation hardness until the system design was proven.

\subsection{ARM20 Mechanical Development}

The ARM20 mechanicals include input and output bearings, input and output position sensors, input brake, gear train, brushless DC motor, wire coil, housing and miscellaneous machined components.

The motor is a frameless custom wound motor with the design parameters specified in Table 7. The manufacturer incorporated Hall sensors for commutation and a $100 \Omega$ RTD sensor, for determining the winding temperature, into the motor's stator windings. The motor magnets were permanently mounted on a custom designed hollow rotor shaft. The hollow shaft allowed for the passage of the wire coil tube. The varnish used on the windings is radiation resistant per 

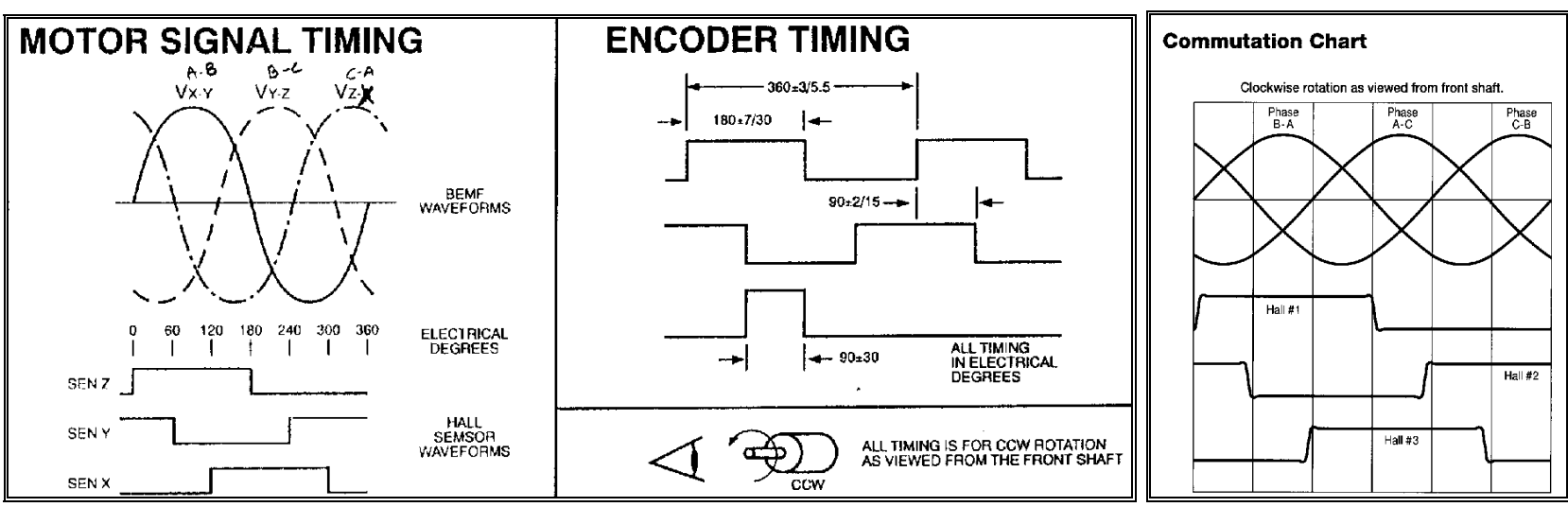

Figure 8: Motor commutation diagrams for a) an MFM motor [MFM, 1998] and b) a Parker Hannifin motor [Parker, 1996].

conversations with the manufacturer. The effect of radiation on the RTD and Hall sensors is unknown.

As there is no industry standard, ARM has chosen to define the Hall configuration with respect to the three motor phases, such that the motor shaft turns clockwise when facing the drive shaft (rotor) as follows: $0 \times 1$ if the corresponding Hall lines up with phase $A B$ back ElectroMotive Force (EMF), $0 \times 5$ if it is 180 out of phase with winding phase AB back EMF (i.e., -phase $\mathrm{AB}$ or phase $\mathrm{BA}), 0 \times 2$ if the corresponding Hall lines up with phase BC back EMF, 0x6 if it is 180 out of phase with winding phase BC back EMF (i.e., -phase $\mathrm{BC}$ or phase $\mathrm{BC}$ ), and 0x3 if the corresponding Hall lines up with phase CA back EMF, 0x7 if it is 180 out of phase with winding phase CA back EMF (i.e., -phase CA or phase AC). The Hall Configuration Number is then comprised of the three Hall numbers in their order in a hexadecimal number with Hall $\mathrm{A}$ being the left most number, Hall $\mathrm{B}$ being the middle number and Hall $\mathrm{C}$ being the right most number as follows: (Hall A)(Hall B)(Hall C) Hex. Many companies number the Halls differently (Halls X, Y, and Z in Figure 8 (a) and Halls 1, 2, and 3 in Figure 8 (b)). In these instances, Hall A would correspond to lowest ordered identifier (i.e., Hall X or Hall 1) and Hall $\mathrm{C}$ will correspond to the highest ordered identifier. For example, the commutation chart in Figure 8 (a) will produce a Hall configuration number of 0x675 (Note the back EMF waveforms are negative because they are produced in the $\mathrm{CCW}$ direction as viewed from the front shaft) and the commutation chart in Figure 8 (b) will produce a Hall configuration number of 0x567 (note these wave forms are also negative because they are with respect to $\mathrm{AC}, \mathrm{CB}$, and $\mathrm{BA}$ ). These numbers are then programmed into the DISC ${ }^{\text {TM }}$ via IDN 32823 such that the DISC $^{\text {TM }}$ can connect to any motor and be configured for commutation without 'guessing' the relationship between the motor windings and the Hall signals.

The actuator gear train technology chosen was the harmonic drive. This topology was chosen because of its zero backlash, compact size and high gear ratios. The model chosen for the ARM20 was a customized version designed to incorporate the output bearing structure. The customization allowed ARM to design a significantly more compact actuator than would otherwise have been possible using a commercial off-the-shelf solution. The ratings for the gear train are given in Table 8.

The ARM20 input section and the PSI20 housings were obtained from a single extrusion. The housings were machined to accommodate a pass through pneumatics connections, mounting 


\begin{tabular}{|l|c|c|}
\hline \multicolumn{1}{|c|}{ Parameter } & Value & Units \\
\hline \hline Rotor Inertia & $1.93 \times 10^{-5}$ & $\mathrm{~kg} \cdot \mathrm{m}^{2}$ \\
\hline Gear Ratio & $80: 1$ & Cycles/Cycle \\
\hline Accuracy & 1.5 & Arc $\cdot M i n$ \\
\hline Repeatability/Lost Motion & 42 & Arc $\cdot$ Sec \\
\hline No Load Starting Torque & 0.04 & $\mathrm{~N} \cdot \mathrm{m}$ \\
\hline Viscous Drag Torque & $2.3 \times 10-5$ & $\mathrm{~N} \cdot \mathrm{m} /(\mathrm{rad} / \mathrm{sec})$ \\
\hline Continuous Rated Load Torque & 47 & $\mathrm{~N} \cdot \mathrm{m}$ \\
\hline Peak Load Torque & 74 & $\mathrm{~N} \cdot \mathrm{m}$ \\
\hline Rated Input Speed (Grease) & 367 & $\mathrm{rad} / \mathrm{sec}$ \\
\hline Peak Input Speed (Grease) & 420 & $\mathrm{rad} / \mathrm{sec}$ \\
\hline
\end{tabular}

Table 8: ARM20 gear train specifications.

holes for the PSI20 shell, input electrical connector, output housing interface and end plate. The housing also contains fins to aid in the dissipation of heat from the motor and PSI20 power electronics. The thermal dissipation constant $\left(\mathrm{R}_{\theta}\right)$ for the shell to ambient is estimated to be around $2.1 \mathrm{Watts} /{ }^{\circ} \mathrm{C}$.

The bearings and seals for the ARM20 were selected such that they could be purchased off-the-shelf. The input bearings have a total moment of inertia of $5 \times 10^{-6} \mathrm{~kg} \cdot \mathrm{m}^{2}$ and a limiting speed of $1570 \mathrm{rad} / \mathrm{sec}$. The total drag torque for the input bearings is less than $0.007 \mathrm{~N} \cdot \mathrm{m}$. The output bearing housing is a proprietary compact arrangement. Its drag torque reflected to the input was empirically found to be approximately $0.01 \mathrm{~N} \cdot \mathrm{m}$ and its mass moment of inertia reflected to the input is approximately $2 \times 10^{-6} \mathrm{~kg} \cdot \mathrm{m}^{2}$. The output bearing arrangement is designed to handle $20,000 \mathrm{~N}$ axial force, $1600 \mathrm{~N}$ radial force and an overturning moment of 210 $\mathrm{N} \cdot \mathrm{m}$ for an L-10 life rating. The ARM20 housing incorporates many static seals and only two dynamic seals. The static seals are designed to keep the housing waterproof and maintain the pneumatic seal throughout the housing. The dynamic seals are located between the input and output housings. They are designed to perform two functions: seal the housing from outside particulates and maintain a pneumatic seal across the housing sections. The seal material selected for the bearings and seals is not radiation resistant but comparable solutions exist as a cost of approximately ten times the value of the seals used. In addition to the seals, a thermal barrier between the motor housing and the PSI20 shell was also designed and fabricated. The purpose of the thermal barrier is to thermally isolate the motor housing from the PSI20 housing.

The brake design went through several iterations during the design process. The first iteration yielded a design that closely resembled a traditional disk brake. This concept was prototyped to incorporate the brake coil, resolver housing, and input shaft bearing. This design was deemed infeasible since the electronics for the resolver and the resolver itself resulted in a more costly design than was acceptable. This design concept can be included in future designs but it is very expensive. The second design utilized a pin in disk concept, which combined a slotted disk with a solenoid. The braking material used for this device was cork. The brake disk was then pre-loaded such that it would break free if a load of more than $30 \mathrm{~N} \cdot \mathrm{m}$ was applied to the brake disk. The brake also included a switch to sense when the brake solenoid was disengaged from the disk. The switch allows a controller to reduce the power applied to the solenoid once it is disengaged from the disk. This results in a significant reduction in the power 
(heat) dissipated by the brake. There is a mechanical brake release built into the motor housing. It is operated by turning a $1.5 \mathrm{~mm}$ Allen wrench clockwise to disengage the solenoid from the disk. In addition to being a brake release, the release is designed to perform as a pop-off valve in case the pneumatic pressure inside the actuator becomes too great.

As mentioned above, the input sensor was initially planned to be a resolver. However, the cost of integrating the sensor into the system was prohibitive from both cost and design volume aspects. Consequently, ARM chose to incorporate the highest resolution encoder commercially available that would fit in the input section of the ARM20 at a reasonable cost. The resolution was 1024 counts before quadrature. This yields a resolution on the output of one count in 327,680 or four arc-seconds after quadrature, assuming a perfect gear train. The inertia of the encoder wheel is negligible. The wheel does include a $90^{\circ}$ index pulse. The encoder head is specified to operate over a temperature range of $-40{ }^{\circ} \mathrm{C}$ to $140{ }^{\circ} \mathrm{C}$ with a maximum guaranteed count frequency of $50 \mathrm{kHz}$. For the above resolution, this yields a maximum input shaft angular velocity of $307 \mathrm{rad} / \mathrm{sec}$ or $2930 \mathrm{RPM}$.

The output sensor is a proprietary sensor developed by ARM to coarsely sense the output position. This sensor is calibrated using a proprietary Fast Fourier Transform (FFT) algorithm to increase the accuracy of the sensor to less than 0.7 degrees and is aligned with the index pulse on the input sensor. This allows the DISC TM to precisely know the output position by homing to the nearest index pulse, which can be found in less than 4.5 degrees for the ARM20. The output sensor has a range of $-270^{\circ}$ to $+270^{\circ}$. This range was determined during simulation such that maximized a manipulator's workspace and dexterity.

The wire coil and connectors were designed as an integral component of the overall mechanical and electrical design of the system. In order for modular robotics to work, the wire harness must be designed to pass through the system with the necessary power drops 'teeing' off the harness. For the overall architecture, ARM chose to pass a twelve (12)-wire harness through the system. On the proximal side (side nearest the PSI20), the wire functions are motor power, motor power return, $24 \mathrm{~V}$ bus, $24 \mathrm{~V}$ bus return, earth ground, end effector power, end effector return, data channel A from system controller, data channel B from system controller, data channel A from DISC ${ }^{\mathrm{TM}}$, data channel B from DISC ${ }^{\mathrm{TM}}$ and data shield. On the distal side of the actuator module, all wire annotations are the same except data channel A from DISC ${ }^{\mathrm{TM}}$, and data channel B from DISC ${ }^{\text {TM }}$ become data channel A to DISC ${ }^{\text {TM }}$, and data channel B to DISC ${ }^{\text {TM }}$, respectively. The wire harness' data communications is designed such that the system controller communicates in a token ring format with the outer most actuator receiving the token first, and the base actuator receiving the token last. This approach maintains the compatibility with the SERCOS standard. The motor power bus is rated to operate over a voltage range of $40 \mathrm{~V}$ to 310 $\mathrm{V}$. The current capacity of the distal connectors for all modules is rated to handle a minimum of four actuators of the same size. The power requirements of the next smaller actuator are specified to be about $1 / 4$ of the previous larger actuator. This allows three actuators of the same size to be integrated before that system will need to step down to the next size of actuator.

The final components to discuss involve the output and input interface plates. Much thought was given to the granularity of modularity in the design of the actuator. Based on past experience, review of past modular design concepts ([Black, 1998]), and input from the DOE personnel and industrial partners, ARM chose to place the quick connect interface in the link rather than in the actuator and design with a common electro-mechanical interface on the input and output sides of the actuators, yokes and links. The bolted interfaces still include the same 


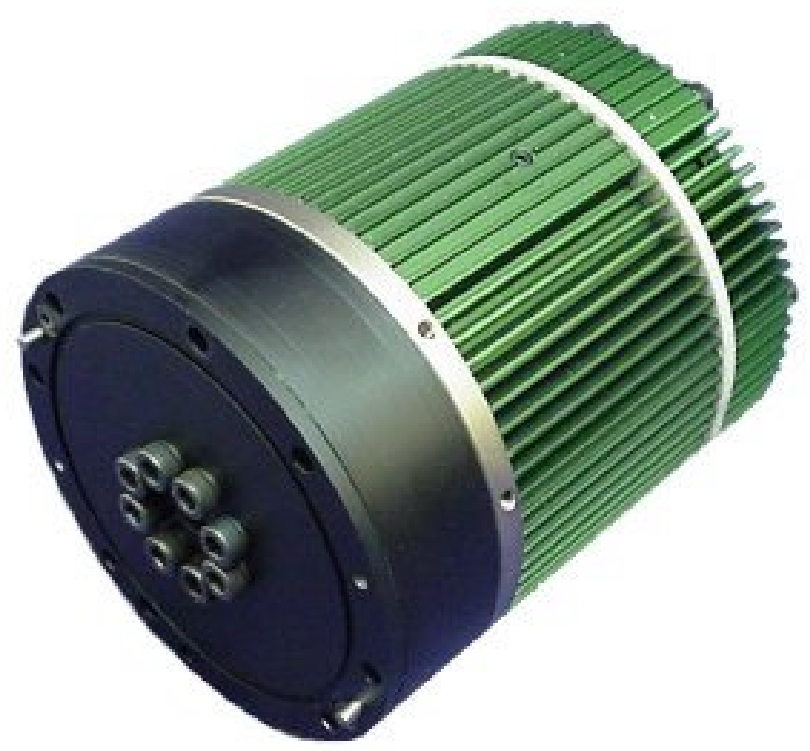

Figure 9: Assembled ARM20 actuator module.

electrical and mechanical aspects of the quick-connect interface but they are mated through bolts rather than some mechanical locking mechanism such as a V-Band. This allowed the actuator to be bolted to either a link or a yoke and greatly facilitated reconfiguration without an increase in design complexity or cost. Consequently, the electronics side is open until it is interfaced with either a yoke plate or link, which reduces the overall weight of the actuator module. More discussion on this issue will be given in the manipulator development and testing section.

Figure 9 shows an ARM20 assembled actuator. Table 9 summarizes the final actuator design specifications and Figure 10 depicts the designed Torque-Speed curve. These specifications are based on a composite of the above specifications for each of the individual components. The continuous output torque is limited by both the continuous current rating of the PSI20 of 1.05 amps, and the continuous torque rating of the gear train. The peak torque rating of the gear train limits the actuator's peak torque rating. The measured specifications are shown in Section 2.2.1.4.

\begin{tabular}{|l|c|c|}
\hline & ARM20 (initial) & ARM20 (final) \\
\hline Performance: & & \\
\hline - Range of Motion & $\pm 340^{\circ}$ & $\pm 270^{\circ}$ \\
\hline - Peak Output Speed & $30 \mathrm{RPM}$ & $36 \mathrm{RPM}$ \\
\hline - Peak Output Torque & $82 \mathrm{~N} \cdot \mathrm{m}$ & $74 \mathrm{~N} \cdot \mathrm{m}$ \\
\hline - Continuous Torque & $40 \mathrm{~N} \cdot \mathrm{m}$ & $47 \mathrm{~N} \cdot \mathrm{m}$ \\
\hline - Bearing Load Capacity (axial/radial) & $3330 \mathrm{~N} / 1500 \mathrm{~N}$ & $20,000 \mathrm{~N} / 1600 \mathrm{~N}$ \\
\hline - Bearing Overturning Load (L10 Life) & $333 \mathrm{~N} \cdot \mathrm{m}$ & $210 \mathrm{~N} \cdot \mathrm{m}$ \\
\hline Physical: & & $115 \mathrm{~mm}$ \\
\hline - Length & $128 \mathrm{~mm}$ & $97 \mathrm{~mm}$ \\
\hline - Diameter & $97 \mathrm{~mm}$ & $2.42 \mathrm{~kg}$ \\
\hline - Mass & $2.3 \mathrm{~kg}$ & \\
\hline
\end{tabular}




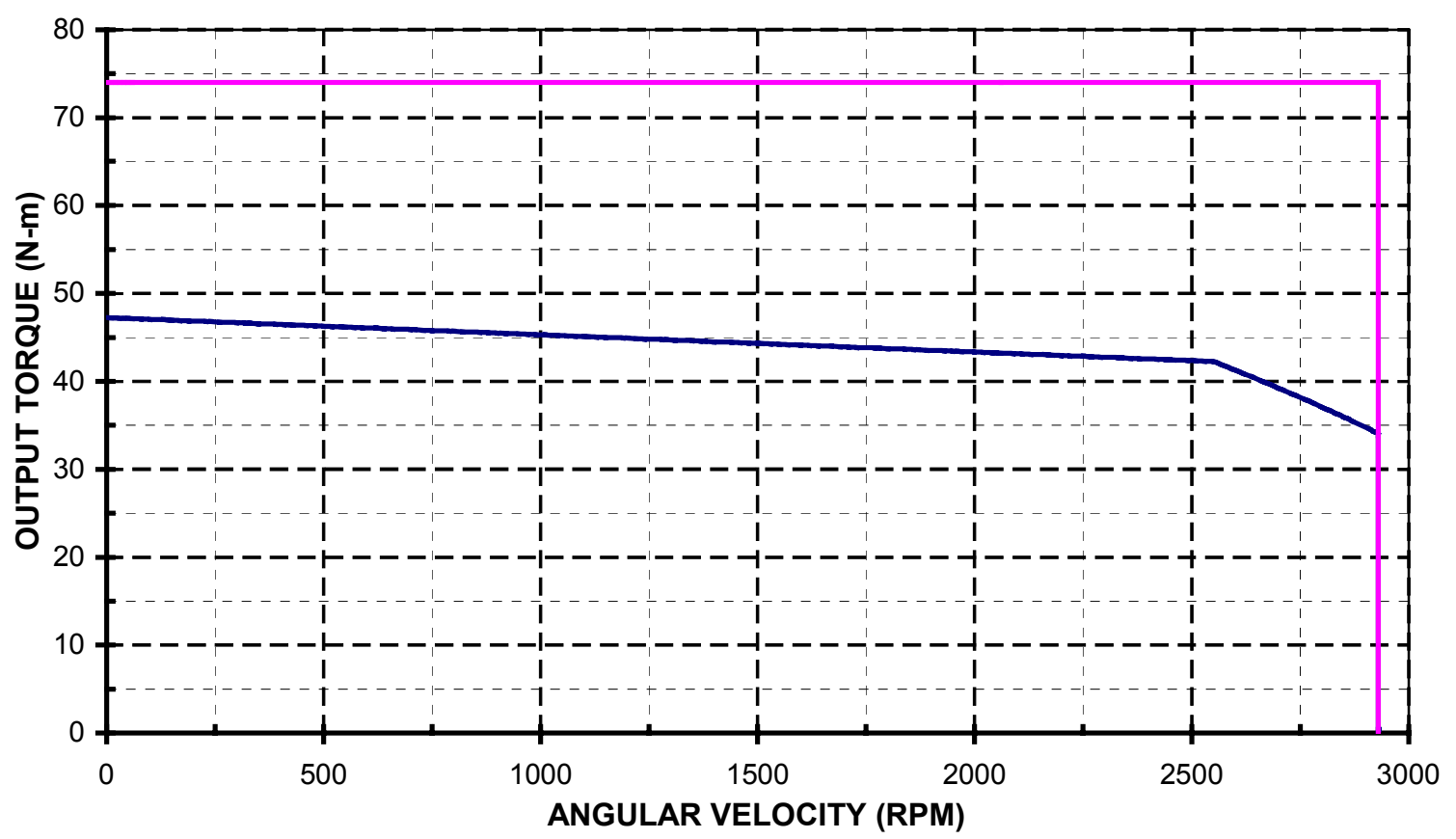

Figure 10: ARM20 designed torque-speed curve.

\subsection{Development}

The CCI development encompassed the selection and design of the control and communications system for the DISC ${ }^{\mathrm{TM}}$. The CCI board contains the main processor, the communications controller, the CPU management, the interface logic, the PSI interface, the debugger/daughter board interface, analog circuitry management, global registration, and serial EEPROM. Each of these aspects will be discussed in the paragraphs that follow. The goal of the DISC $^{\text {TM }}$ was to aim for a minimum peak ambient operating temperature of $85{ }^{\circ} \mathrm{C}$. Thus, the temperature rating of all components on the CCI must exceed $85{ }^{\circ} \mathrm{C}$. The maximum diameter constraint was set to $60 \mathrm{~mm}$. The overall thickness of the board excluding connectors was to be less than $9.5 \mathrm{~mm}$. The expansion connector to the PSI should contain less than 80 pins. The daughter board should include at a minimum capability for handling the following: encoder input, home plus two limit inputs, SSI/SPI and SERCOS communication interfaces and the processor debugger interface. The MTBF design goal for the DISC ${ }^{\mathrm{TM}}$ was set to be greater than 15,000 hours. This goal combined with the peak ambient temperature precluded the use of electrolytic capacitors in the design of both the CCI and PSI boards. All capacitors topologies used on the boards are fully specified over the temperature range of $-40{ }^{\circ} \mathrm{C}$ to $125^{\circ} \mathrm{C}$. All resistors were sized such that their maximum power dissipated at $125^{\circ} \mathrm{C}$ was below their rated maximum power dissipation at $125^{\circ} \mathrm{C}$.

For the controller, ARM researched the most current embedded 16-bit and 32-bit microcontrollers and DSPs on the market in 1998. These processors were compared against an embedded processor that ARM had utilized in a prior generation of design. In order to maximize design life, ARM selected a processor that was just being released in 1998. In hindsight, this decision led to approximately 9 months delay in the system development including both software and hardware development. The biggest contributors to this delay were related to the 3 month 
delay in the release of the development system, the need to design and fabricate an interface to the development system, and the acquisition of the processors themselves as they were put on allocation in early 1999 with ARM receiving half their allotment in August 1999 and their remaining allotment in November 1999. In addition, ARM had to redevelop a major portion of the embedded software system due to a switch from an earlier processor architecture. In addition, the code for operating the DISC ${ }^{\text {TM }}$ resides within the processor's internal Read Only Memory (ROM).

Communication protocols reviewed as possible candidates for the DISC ${ }^{\text {TM }}$ include: SERCOS, HDLC, Firewire, ControlNet, Macro, Mil STD-1553B, Fast Ethernet, ARCNET, and Profibus DP. Of these buses, the only deterministic ones included ARCNET, Profibus DP, Firewire, and SERCOS. Of these choices, SERCOS was implemented since it was (and still is) the only protocol standard defined for motion control and it could be implemented on a single chip, which was rated over the industrial temperature range. The fact that it is a standard defined for motion control allowed ARM to expedite the development of the communications interface. This development was aided by the off-the-shelf purchase of a SERCOS slave development kit, a SERCOS bus monitor, and a SERCOS master for controlling the DISC ${ }^{\mathrm{TM}_{\mathrm{S}}}$ for the ARM20 and ARM32. The choice of implementing the communication's protocol using SERCOS is estimated to have saved three months of development time since the framework for SERCOS was purchased off-the-shelf.

The CPU management circuitry included the design of the $5 \mathrm{~V}$ to $3 \mathrm{~V}$ circuitry, the reset management, power and debug LEDs, and system clock. The $3 \mathrm{~V}$ bus is derived from the $5 \mathrm{~V}$ bus and the reset circuitry operates off $3 \mathrm{~V}$ to insure that the $3 \mathrm{~V}$ bus is stable before the CPU comes out of reset. The CCI board contains a dual SMT LED whose colors are red and green. The red LED is illuminated when $5 \mathrm{~V}$ power is applied to the board. The green LED is toggled on and off by an $\mathrm{I} / \mathrm{O}$ pin on the processor. It is used to facilitate debugging of the embedded code.

The interface logic consists of a programmable Complex Programmable Logic Device (CPLD) and 3 to 5 volt data bus voltage leveling logic. The CPLD handles all of the chip interface logic and I/O signals associated with the CCI as well as some 3 to $5 \mathrm{~V}$ signal leveling since the CPLD has $5 \mathrm{~V}$ tolerant inputs with programmable level outputs. The CPLD is programmed with ARM's proprietary algorithm.

Overall, at the onset of the design of the CCI board, ARM decided to incorporate into the CCI support for interfacing either via the PSI or Daughter Board interface to the following suite of sensors: Incremental encoder, three-phase current sensors, voltage detection, various temperature sensors, limit switches, a home switch, a torque sensor, and resolver. Thus, the PSI interface contains the following capability: an address bus, an 8-bit data bus, a dedicated chip select, five programmable I/0 signals, a quadrature encoder interface with index pulse, Hall inputs, commutation outputs, differential SERCOS signals, two dedicated interrupts, PWM enable, three independent PWM channels, SPI interface with two dedicated chip selects, Main Bus Power Detect, clock filter signal, and 10 channels of analog inputs. The daughter board interface includes an interface for programming and debugging the DISC ${ }^{\text {TM}}$ 's embedded software, two SPI channels, quadrature encoder with index input, SERCOS signals for interfacing to a fiber optics driver, two CAN channels, three general purpose I/Os.

The analog circuitry management handles the analog interface to the controller on the CCI board. This circuitry provides a $4.5 \mathrm{~V}$ reference to the analog to digital (AtoD) converter. It 


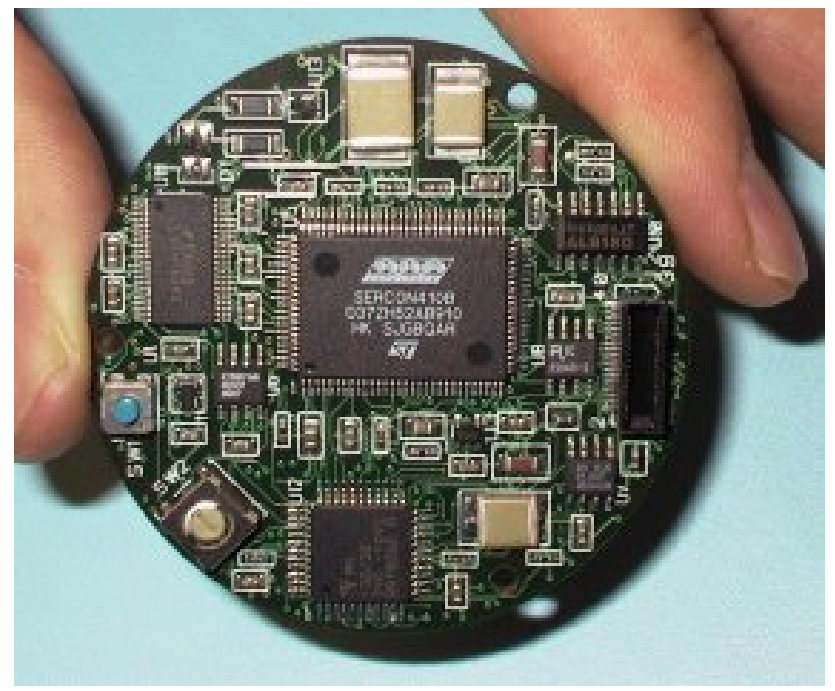

Figure 11: Populated CCI board.

also incorporates two temperature sensors (one for the top of the board and one for the bottom of the board) with a linear range of $-50^{\circ} \mathrm{C}$ to $125^{\circ} \mathrm{C}$ and an accuracy of $+/-1^{\circ} \mathrm{C}$.

The final two circuits to be discussed are the global registration and the serial EEPROM. The global registration is a 4-bit, 16-position selector switch. This four-bit nibble is the concatenated with a board registration number from the PSI board to form a unique address for the DISC ${ }^{\text {TM }}$. This will allow 16 of the same devices (i.e., 16 ARM20's or 16 ARM32's) to be connected on the same ring. The serial EEPROM is a 512 byte EEPROM that is used to store volatile operating variables on the DISC ${ }^{\mathrm{TM}}$.

Figure 11 shows a finished CCI board. The final diameter of the board is $58 \mathrm{~mm}$. The thickness of the components excluding the board-to-board connectors is less than $10 \mathrm{~mm}$. All components on the board are rated for a maximum temperature of $85{ }^{\circ} \mathrm{C}$ with all but five components rated for a peak temperature of more than $105^{\circ} \mathrm{C}$. Most of the components that are rated at $85{ }^{\circ} \mathrm{C}$ are for the SERCOS communications system. The 8-layer board contains over 100 buried resistors and over 480 micro-vias. There are over 110 components and 212 connections. Table 10 compares CCI's preliminary design specifications to the final specifications. One can observe from this comparison that this design met or exceeded every design goal set in the preliminary design. To date, the only design error, on the CCI found during testing, is a signal associated with the processor coming out of reset when it is not connected to the debugger. This signal is presently pulled high instead of low. This error stemmed from an undiscovered revision of the original processor specification from it preliminary specification. Fortunately, this line is accessible through the daughter board interface. Thus, by placing a mating connector in the socket and pulling the appropriate line to ground, ARM was able to overcome this issue. The other design changes that ARM would recommend to incorporate in future revisions include a method to force the processor to boot using its default configuration, an increase in the EEPROM size to accommodate more parameters, and additional circuitry to handle voltage spikes/power surges across the RS-485 SERCOS line drivers.

In addition to the CCI board, ARM needed to design and fabricate three boards for prototyping and testing the CCI board. The first of these boards was a daughter board which 


\begin{tabular}{|c|c|c|}
\hline Parameter Specified & CCI (initial) & CCI (final) \\
\hline - Temperature Range (Min. for all comp.) & $-40<\mathrm{T}<85^{\circ} \mathrm{C}$ & $-40<\mathrm{T}<85^{\circ} \mathrm{C}$ \\
\hline - Life (MTBF) & 15,000 Hrs. & $>15,000 \mathrm{Hrs}$ \\
\hline - Outside Diameter & $66 \mathrm{~mm}$ & $58 \mathrm{~mm}$ \\
\hline - Thickness excluding connectors & $12 \mathrm{~mm}$ & $10 \mathrm{~mm}$ \\
\hline - Voltage Input & $5 \mathrm{~V}, 3.3 \mathrm{~V}$ & $5 \mathrm{~V}$ only \\
\hline - Total Power Consumption (operating) & $<3 \mathrm{~W}$ & $<2 \mathrm{~W}$ \\
\hline - High Speed Communications Bus & SERCOS & SERCOS \\
\hline - Device Level & DeviceNet & 2 CAN buses avail. \\
\hline - Sensor Level & SSI & 2 SSI Channels avail. \\
\hline - System Clock Frequency & $40 \mathrm{MHz}$ & $32 \mathrm{MHz}$ \\
\hline - SRAM & 16 Kbytes & 26 Kbytes \\
\hline$\cdot$ ROM & 128 Kbytes & 512 Kbytes \\
\hline - Expansion Connector & Less than 40 Pins & 40 Pins \\
\hline - PSI Board To Board Connector & Less than 80 Pins & 80 Pins \\
\hline
\end{tabular}

Table 10: Comparison of CCI's preliminary specifications to its final specifications.

mates with the processor's evaluation board that was purchased off-the-shelf. The processor was so new that the evaluation board did not have a daughter board available off-the-shelf. The daughter board was used for accessing the signals from the processor to facilitate prototyping the entire CCI and PSI20 designs before the boards were fabricated and populated. This also allowed for simultaneous software and hardware co-design of the DISC ${ }^{\mathrm{TM}}$. The other two boards designed by ARM were a CCI daughter card and a PSI break out board (See Figure 12). The $\mathrm{CCI}$ daughter card is used for connecting the debugger to the process and for programming the processor. The PSI break out card was used to interface the CCI board to the PSI20 prototype board. This facilitated the testing of the CCI board before the PSI20 design was complete.

\subsection{PSI20 Development}

The PSI20 was developed to drive the ARM20 modular actuator. The PSI20 has four main sub-systems: $\mathrm{CCI}$ and external interfaces, sensor interface logic, power interface logic, and

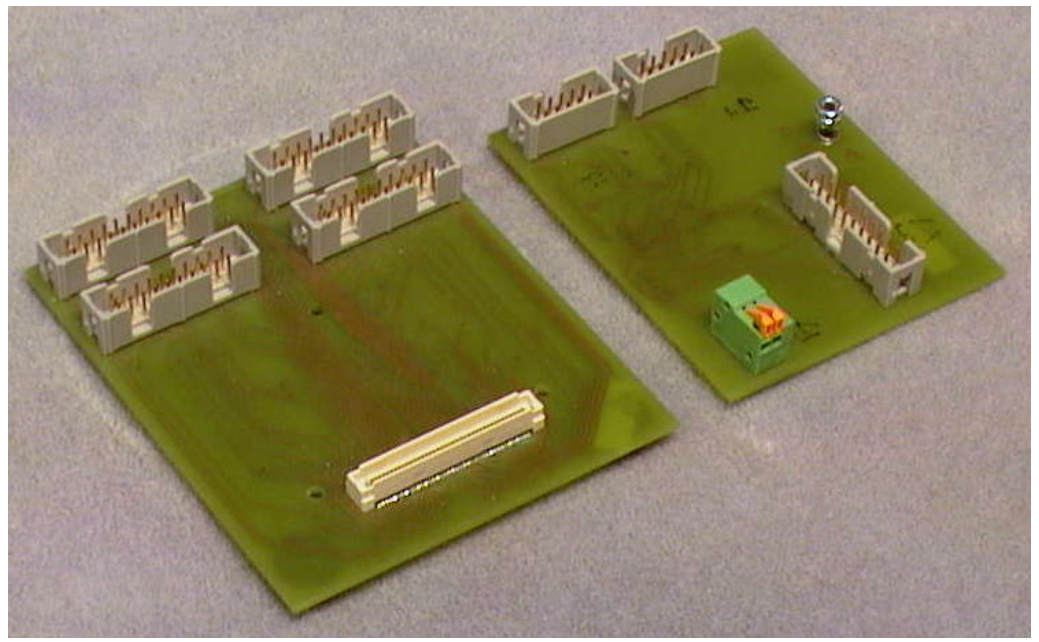

Figure 12: PSI break out board (left) and CCI daughter board interface board (right). $D E^{\prime}-A C^{\prime 2} 26-98 F^{\prime \prime} 140371$ 


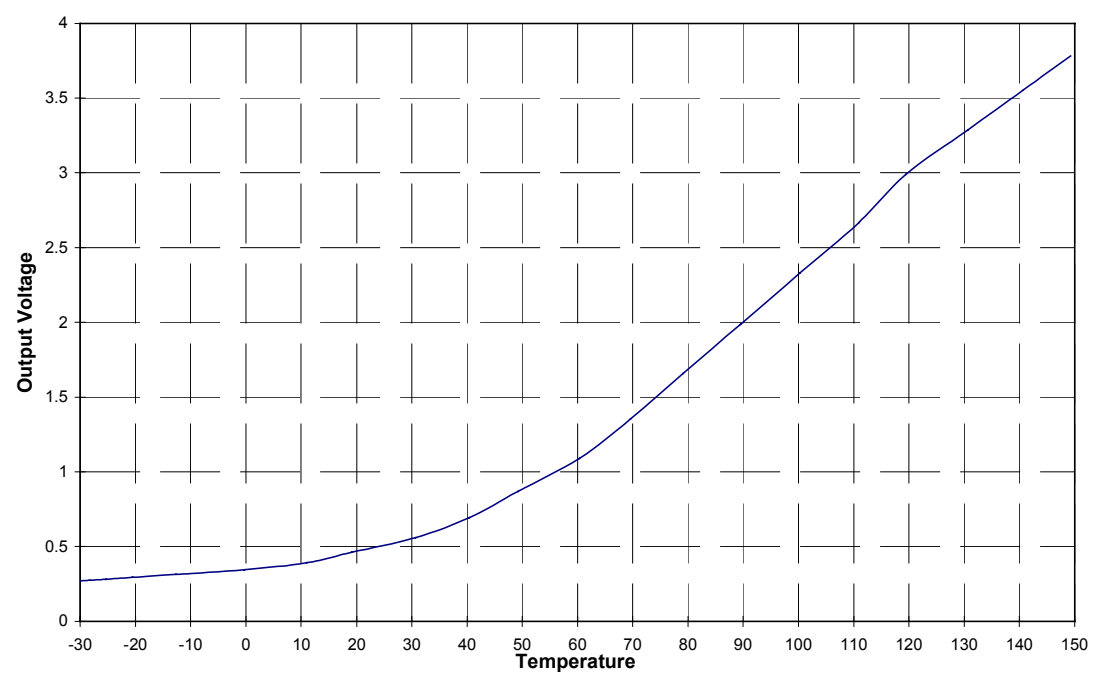

Figure 13: PSI20 thermistor circuit output voltage versus temperature.

power conversion and monitoring circuitry. The PSI20 was designed with the same environmental constraints as the CCI board.

The CCI and external interfaces consists of the CCI interface, the main power bus interface, the absolute position sensor interface, power output interface, motor sensor interface, encoder and brake switch interface, and PSI20 thermistor interfaces. The CCI interface contains the following signals: SERCOS pass through data bus, 3 PWM channels, PWM output enable, 6 commutation lines, quadrature encoder and index signals, 3 Hall signals, commutation select signal, brake enable signal, 6 data lines, address line, address chip select, over-current interrupt, 2 SPI channels, brake PWM signal, current filter clock, main power bus present signal, and 6 AtoD channels for sensing the bus voltage, PSI20 temperature, motor current, transistor's A and $\mathrm{C}$ low side case temperature, and motor temperature. The board connects to the main wire harness through a pigtail. The pigtail has the following 8 signals: motor power and return, $24 \mathrm{~V}$ and $24 \mathrm{~V}$ return, and differential SERCOS data in and out of the DISC ${ }^{\mathrm{TM}}$. The $24 \mathrm{~V}$ bus is fused by a 2 Amp fuse, and the main power bus is fused by a $5 \mathrm{~A}$ fuse. The $5 \mathrm{Amp}$ fuse is necessary to handle surges on the main power bus during startup and shutdown of the motor. The power output interface connects the PSI20 to the brushless DC motor, dynamic braking resistor, and the brake. The motor sensors are the Hall sensors and a RTD sensor integral to the stator winding.

The sensor interface logic conditions the sensor signals before they are sent to the CCI board. This logic handles the following sensors: encoder, motor Halls, temperature including motor RTD, thermistor, and semiconductor topologies, current, absolute position sensor and main bus voltage.

Two thermistors attached to the low side transistors associated with driving motor phases $\mathrm{A}$ and $\mathrm{C}$, are used to sense the PSI20 shell temperature as well as monitor the transistor junction temperature. The thermistor circuits are designed such that they are the most sensitive and linear over the range of 60 to $115{ }^{\circ} \mathrm{C}$. The designed accuracy of the thermistor circuit is $+/-3{ }^{\circ} \mathrm{C}$ over the linear range across multiple PSI20's. Figure 13 shows the theoretical design curve for the thermistor circuit. This is the region where the monitoring of the shell temperature is the most critical for the PSI20. The semiconductor sensor is the same as that used on the CCI board with a linear range of $-50{ }^{\circ} \mathrm{C}$ to $125^{\circ} \mathrm{C}$ and an accuracy of $+/-1{ }^{\circ} \mathrm{C}$. The motor RTD sensor goes 


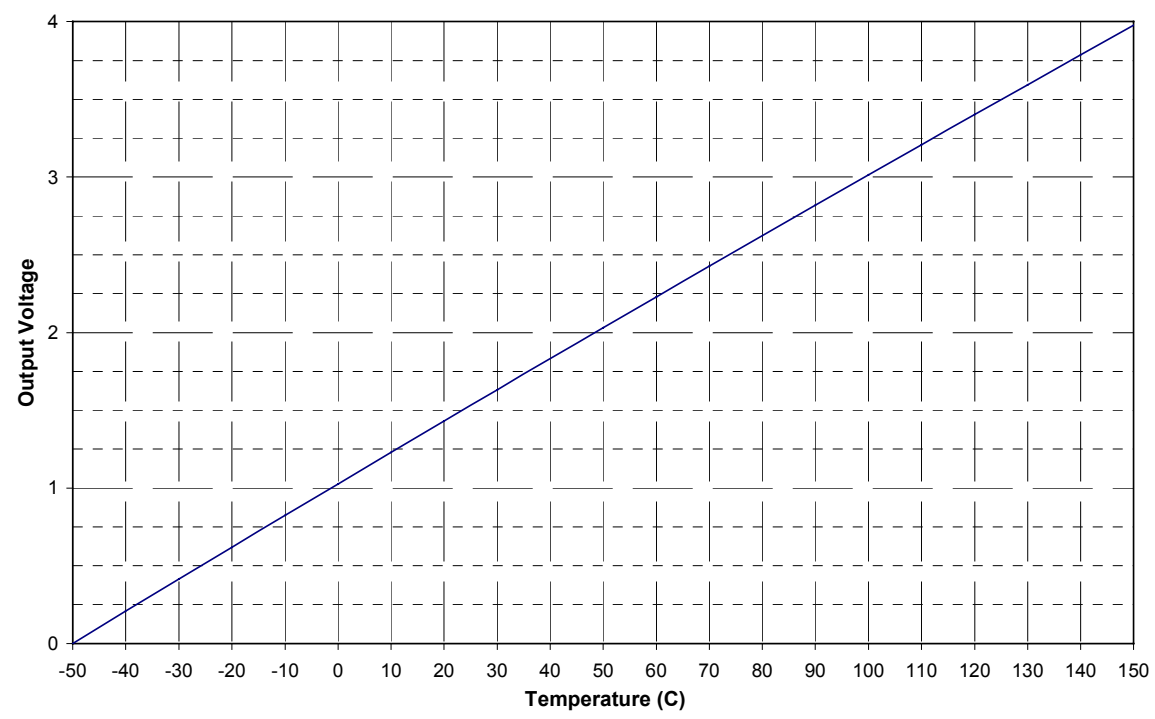

Figure 14: Theoretical design curve for motor RTD circuit.

through a precision operational amplifier circuit with a fixed gain and a low pass filter with a cutoff frequency of $15 \mathrm{~Hz}$. The accuracy of the RTD sensor is better than $+/-2{ }^{\circ} \mathrm{C}$ across multiple PSI20s. Figure 14 shows the theoretical design curve for the motor's RTD temperature sensor circuit.

The current sense circuit is optimized for sensing the motor's current over the range of 0 to 3.5 amps. This provides maximum resolution over the motor's specified current range. Figure 15 shows the designed relationship between the sensed current and the output voltage. The output of this amplifier is filtered by a 2-pole low pass analog filter with a cutoff frequency of $4.8 \mathrm{kHz}$ and an 8-pole Bessel filter with a software selectable cutoff frequency.

The absolute position sensor's conditioning circuitry contains the circuitry for converting the analog signals into a digital signal. The absolute position sensor is then calibrated with respect to the output flange when initiated via a SERCOS procedure. More discussion on SERCOS procedures is given in Section 2.2.1.2. The signals are converted into 16-bit digital signals that are then processed by the controller.

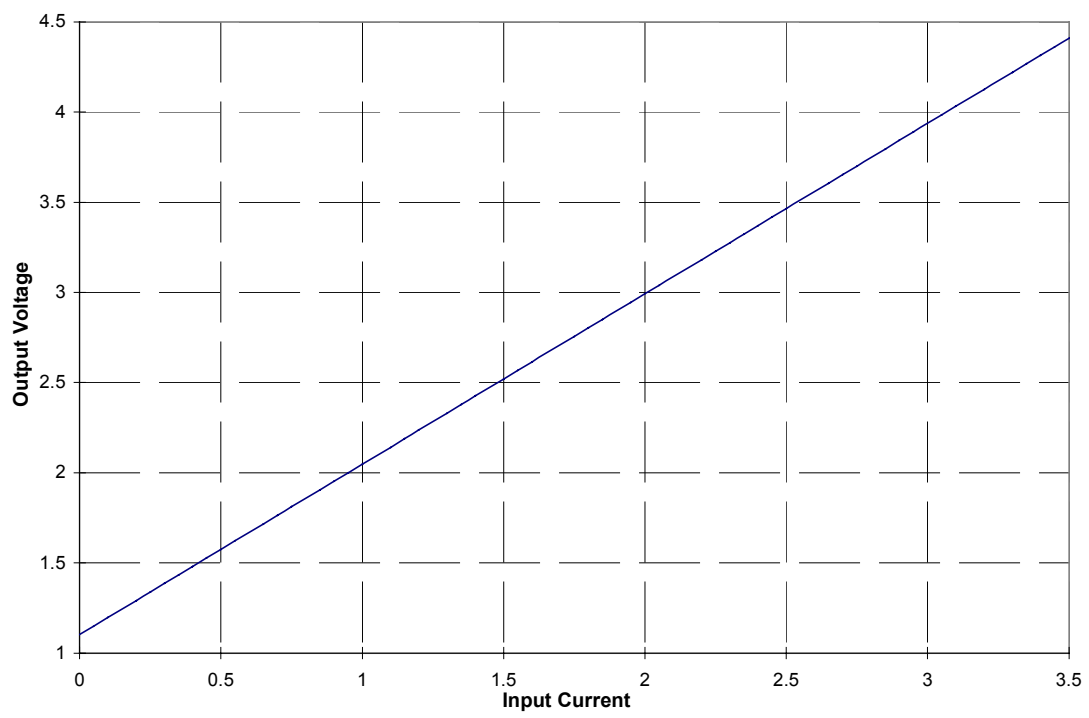

Figure 15: Designed Input motor current versus output voltage curve. 


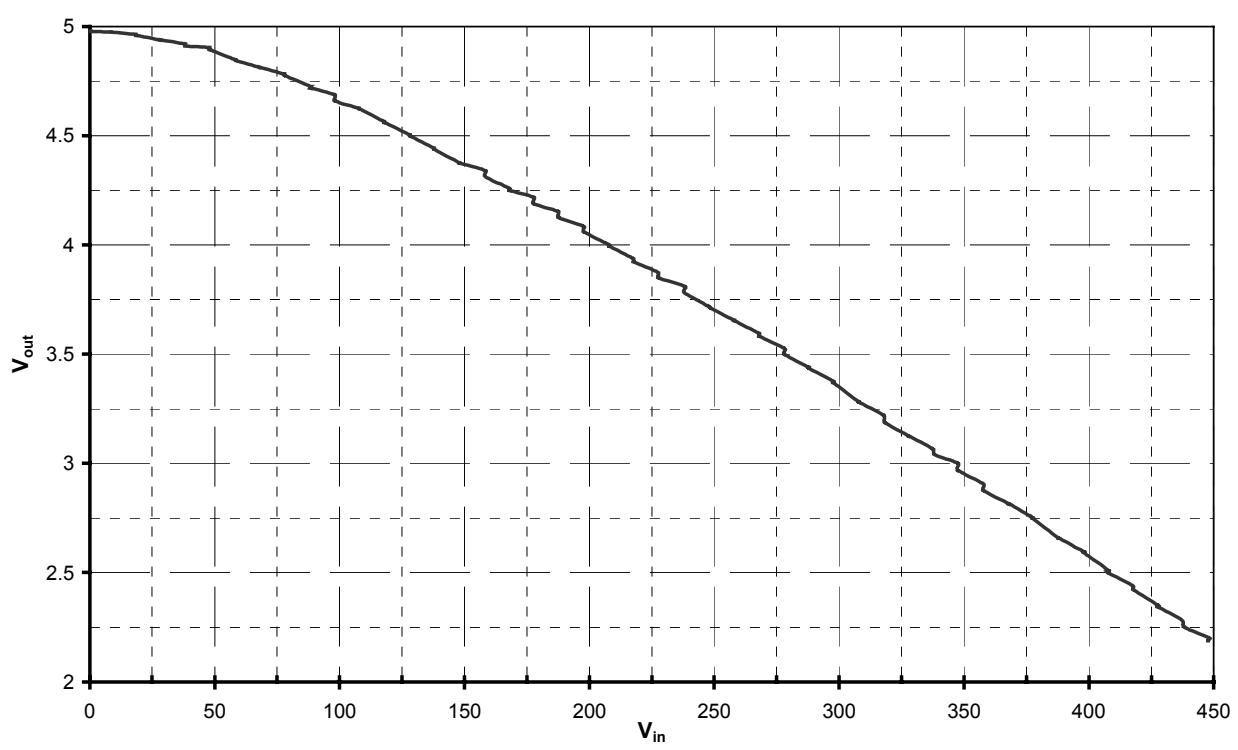

Figure 16: Empirical curve for input bus voltage versus output voltage.

The main bus voltage is sensed using an optocoupler operating its linear region. This circuit produces an output current proportional to the input current which can be kept very small (0-3 ma). This provides greater than 2500 volts isolation from the high voltage circuit while dissipating very little power (less than one watt). Figure 16 shows the relationship of the output voltage to the input voltage. Note that this circuit cannot sense the bus voltage very accurately until it is greater than about $40 \mathrm{~V}$. This output of the circuit is also used by the dynamic braking circuitry in the power interface logic.

The power interface logic contains the following functionality: PWM control, threephase motor transistor driver, motor transistors, and the brake drive. The PWM control functionality is carried out in a CPLD. It handles the functionality for the motor gate drive, the brake gate drive, and the board identity word. The motor gate drive generates the six output transistor drive states based on the input commutation topology, the three PWM states, the PWM enable and the six commutation states. The CPLD allows the PSI20 to commutate the motor in either sinusoidal or trapezoidal modes. The brake gate drive allows full voltage to be supplied to the brake coil until the brake is disengaged. Once the brake is disengaged, the holding current is reduced from about 2 amps to about 0.4 amps, which decreased the power dissipated by a factor of approximately 20. If the brake becomes engaged during operation due to a shock, full power is reapplied across the coil until it is disengaged. The 8 bit board identity word allows the CCI software to decipher the type of PSI board attached to it by identifying the type and size of motor attached, whether it is a DISCActuator ${ }^{\mathrm{TM}}$, a DISCMotor ${ }^{\mathrm{TM}}$ or other type of system, and type of communications medium. This 8-bit word is read by the software during initialization and concatenated with the 4-bit word from the global address switch to form a unique address for the DISC ${ }^{\text {TM }}$. The motor transistor drive circuitry receives the opto-isolated transistor drive signals from the PWM logic. This circuitry also monitors the current on the main power bus for an over-current situation. The circuit is designed to disable the power in approximately $2 \mu \mathrm{s}$. This is significantly faster than the DISC ${ }^{\mathrm{TM}}$ software could sense such a condition and shutdown the transistors. The motor transistor circuitry is a three-phase H-bridge configuration whose transistors are optimally designed to handle 1.05 Amps, continuous current, and 3.0 Amps, peak 


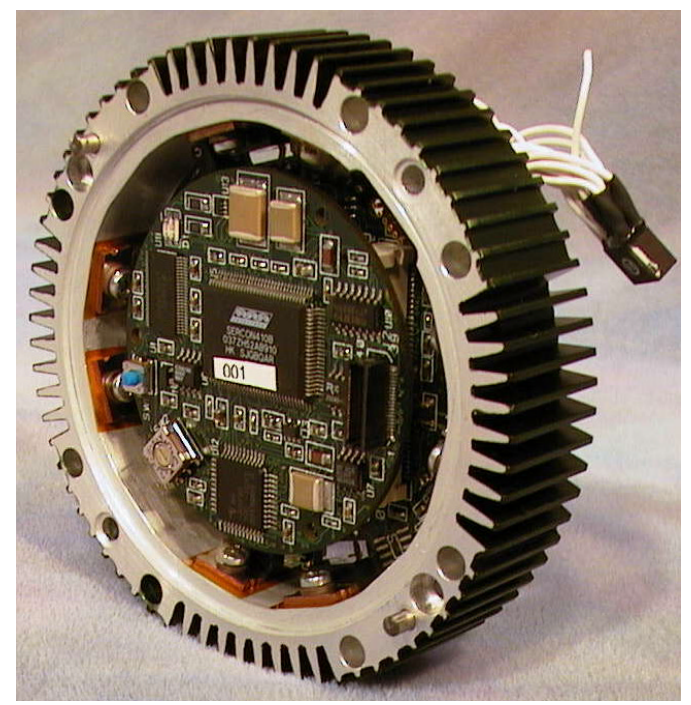

Figure 17: Completed PSI20 board.

current and a bus voltage up to $320 \mathrm{~V}$. The brake drive circuitry is also optically isolated from the logic side of the PSI20.

The power conditioning circuitry generates the $5 \mathrm{~V}$ for the digital and analog circuitry, and the $15 \mathrm{~V}$ for the transistor gate drive circuitry, and dynamically bleeds the main power bus. An off-the-shelf DC-DC converter is used to derive $5 \mathrm{~V}$ of electrically isolated power from the $24 \mathrm{~V}$ bus. The converter can deliver $5 \mathrm{~W}$ over the temperature range of $-50{ }^{\circ} \mathrm{C}$ to $125^{\circ} \mathrm{C}$. The $15 \mathrm{~V}$ is also derived from the $24 \mathrm{~V}$ bus but it is not electrically isolated. The main bus bleed circuit is designed to dynamically brake the motor bus at $412 \mathrm{~V},+/-1 \mathrm{~V}$. This circuit has a hysteresis of approximately 45 volts.

Figure 17 shows a completed PSI20 mounted in the same extrusion that was used for the ARM20 motor housing. The final diameter of the board is $71 \mathrm{~mm}$. The board has 150 passive and active components and 153 nets. The board is laid out in 8 layers and fabricated with $1 \mathrm{oz}$. copper on polyimide material. The board also has 350 through-hole pads and vias. All components on the board are rated for a maximum ambient temperature of $85{ }^{\circ} \mathrm{C}$ with all but four of the components rated for a peak temperature of more than $105{ }^{\circ} \mathrm{C}$. The $24 \mathrm{~V}$ bus draws 0.08 Amps of quiescent current for powering the external sensors and the PSI20 board when it's not connected to CCI board, brake or motor. Table 11 summarizes the PSI20 final specifications.

\begin{tabular}{|l|c|c|}
\hline \multicolumn{1}{|c|}{ Parameter Specified } & Specification & Units \\
\hline \hline - Temperature Range (Min. for all comp.) & $-40<\mathrm{T}<85$ & ${ }^{\circ} \mathrm{C}$ \\
\hline - Life (MTBF) & $>15,000$ & $\mathrm{Hrs}$. \\
\hline - Outside Diameter & 71 & $\mathrm{~mm}$ \\
\hline - Thickness excluding connectors & $12 \mathrm{~mm}$ & $\mathrm{~mm}$ \\
\hline - Operating Voltage Input & 24 & Volts \\
\hline - Total Power Consumption (quiescent) & $<2$ & Watts \\
\hline - Main Bus Voltage Input & $<310$ & Volts \\
\hline - Max. Motor Power Output & 250 & Watts \\
\hline
\end{tabular}




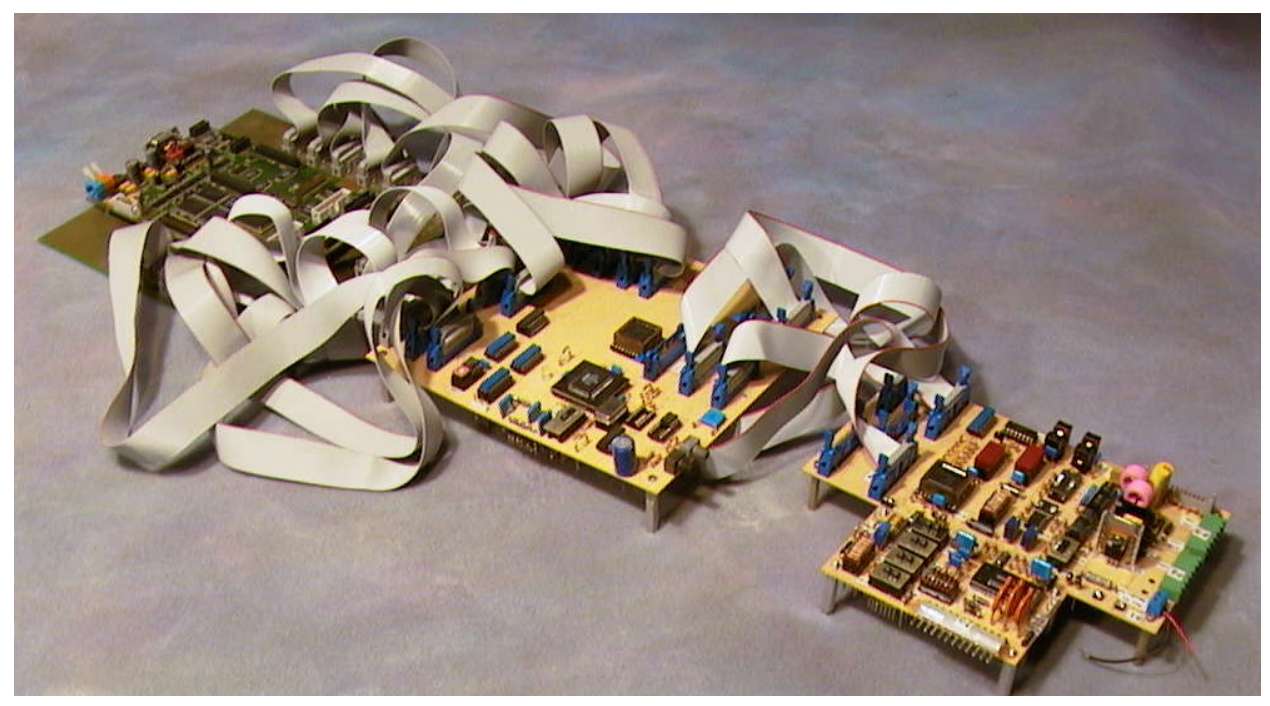

Figure 18: Prototype setup for CCI and PSI20.

Prior to fabrication, the entire PSI20 circuit was prototyped and each of the circuits' functionality verified. Figure 18 shows the PSI prototype connected to the CCI prototype. While the prototypes did aid ARM is solving many design issues with the PSI20, there were still several that it did not address. The first issue related to the current sense shutdown by the gate drive circuit. On the prototype, the circuit was isolated from the motor power leads; however, on the PSI20 Printed Circuit Board (PCB), the traces for Phase A lead and Phase B encircled the sense circuit. Thus, when the current would surge in the system such as during a reversal of the motor's rotor direction or upon a sudden increase in the current command from standstill, the PSI20 would signal that an over-current situation has occurred and would require the circuitry to go through a reset sequence. This problem was partially remedied by modifying the over-current sense circuit on the board. This solved the issue during startup but did not resolve the problem during reversal of shaft direction. The next step taken by ARM was to reduce the rate of turn-on of the transistors by increasing their gate resistance. This effect reduced the EMI generated by the traces. However, the side effect of this design revision was an increase in the amount of heat dissipated by the transistors. After several weeks of experimentation, ARM was able to increase the gate resistance to a point at which no false over-current error occurred. Another side effect of increasing the gate resistance was that the transistors now occasionally either turned off too slow at full voltage or turned on while its complementary transistor was on. Both situations caused a shoot through situation on the motor power bus leading to immediate transistor failure. This problem was overcome by adding a switching diode in the shutdown direction. This gives the effect of a slow turn-on and fast turn-off switching time for the transistor. One final PSI20 problem, occurring in the final circuit layout, was that the Main Bus Detect circuit would occasionally falsely indicate under low voltage $(\sim 100 \mathrm{~V})$ conditions with the rotor locked that the main bus was absent. This problem was a result of the same problem as that for the over-current sense circuit. The resolution to this issue was to form a low pass filter for the bus signal that was input into the comparator used for generating the signal.

For future revisions of the PSI20, ARM will make the necessary changes to remedy the above issues by adding the appropriate gate resistor values with the parallel diode, re-routing Phase A current trace, and revising the over-current sense circuit. In addition to these issues, the 


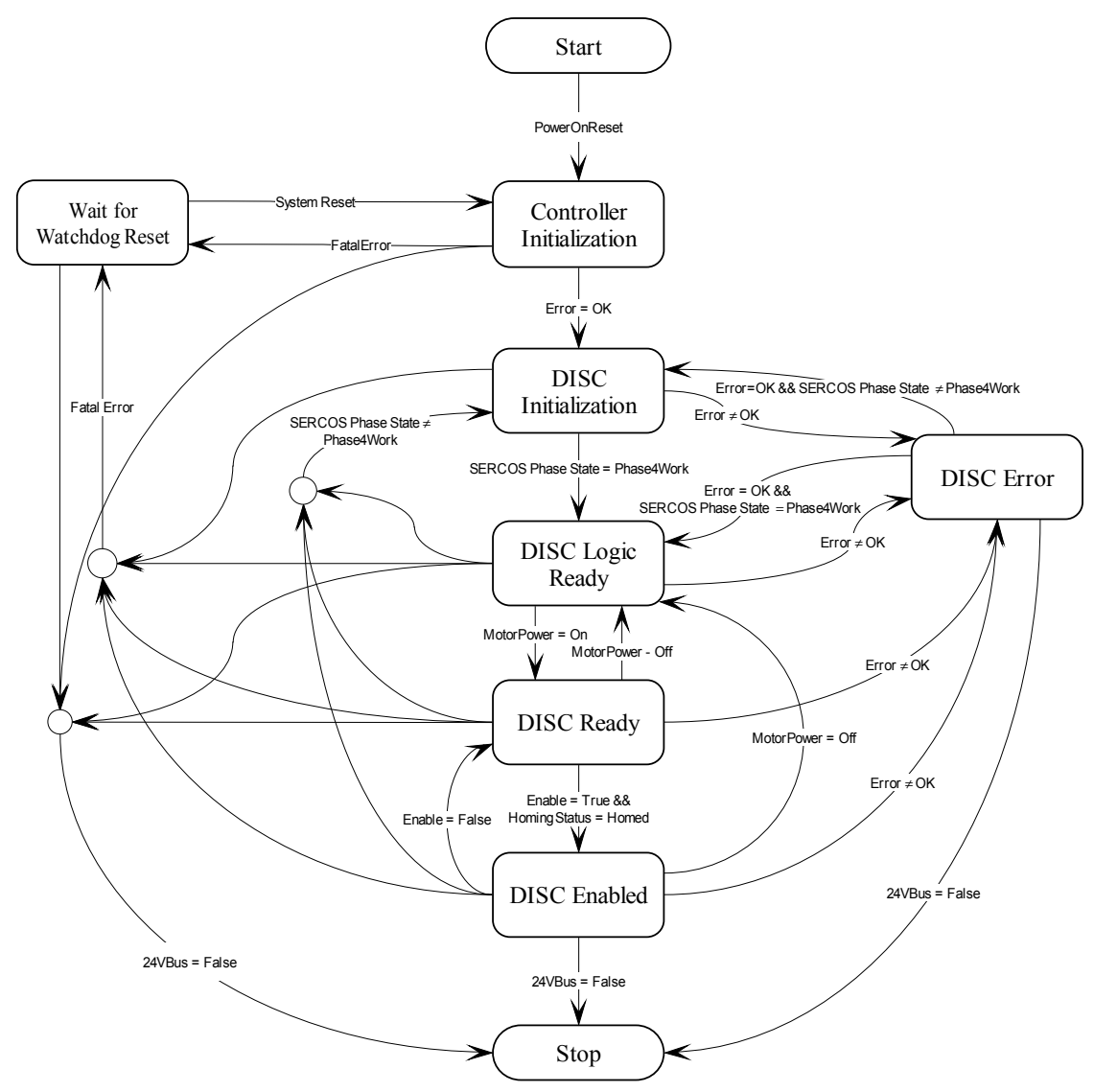

Figure 19: DISC ${ }^{\mathrm{TM}}$ state diagram.

following issues should be addressed by future designs: linearization of the voltage sense circuit, isolation of the current sense circuit from the logic power side, and removal of one of the transistor's thermistors. The linearization of the voltage sense circuit will improve system calibration and software issues. The current sense circuit presently connects the high power bus return to logic ground through a high impedance $(>120 \mathrm{~K} \Omega$ ) fixed gain amplifier. The isolation of the current sense circuit comes about from the fact that when the wire harness is severed (a condition that can happen if one takes apart the quick-connection without removing power from the robot), the main bus tries to release its energy in any manner possible. This can happen through one of three paths it has to ground: its own return, the $24 \mathrm{~V}$ return, or the data bus return. It will use the data bus return when it is the last path to ground. If it uses the data bus return, then the RS-485 driver and the current sense circuit will fail or be significantly overstressed, as this circuitry will have carried significantly more current than originally specified by the manufacturer. The removal of the thermistor is to reduce cost in the overall design.

\subsubsection{DISC ${ }^{\text {TM }}$ Software Development}

Figure 19 depicts the DISC ${ }^{\text {TM }}$ software state machine. This state machine controls the execution of the following modules: boot and initialization software, SERCOS communications, asynchronous routines, and the hard real-time control and interrupt routines and PSI specific routines. With the exception of the SERCOS communications software whose framework was purchased from SICAN, GmbH (now called sci-worx, GmbH), the software design was 


\begin{tabular}{|c|l|l|l|c|}
\hline \multirow{3}{*}{ IDN } & \multicolumn{2}{l|}{ Name (abbreviation) } \\
\cline { 2 - 5 } & \multicolumn{2}{|l|}{ Function/description } & Unit \\
\cline { 2 - 5 } & Length (bytes) & $\begin{array}{l}\text { Minimum input value } \\
\text { Maximum input value }\end{array}$ & Scaling/resolution & \\
\hline
\end{tabular}

Figure 20: Structure of IDN definition [SERCOS, 1998b].

developed from scratch. The operation of this state machine is driven by the SERCOS specification (see [IEC, 1995], [SERCOS, 1998a], [SERCOS, 1998b] for more information on the SERCOS standard), which dictates the transition to all states except Controller Initialization, and Watchdog Reset.

The SERCOS standard is the only communication standard defined for digitally controlled drives. Unlike many other communication protocols such as Ethernet, ARCNet, or ProfiBus DP, this standard defines all seven layers associated with the OSI Reference model: Physical (L1), Link (L2), Network (L3), Transport (L4), Session (L5), Presentation (L6) and Application (L7) layers. ARM implemented the SERCOS standard according to [IEC, 1995] with the exception of the Physical Layer (L1). For the L1 layer, the DISCActuators ${ }^{\mathrm{TM}}$ transmit over an RS-485 twisted pair wired in a token ring format in lieu of fiber optics terminated with FSMA connectors. The reason for going with twisted pair over fiber optics was for costs associated with implementing the electro-mechanical interfaces across the quick-connects and the actuators and potentially low radiation tolerance. The data medium is converted between the standard's L1 specifications and RS-485 in the power supply box. SERCOS defines Identifications Numbers (IDNs) that are used to configure and control the drive. Figure 20 presents the definition of an IDN structure. There are seven elements defined for an IDN: number (element 1), name (element 2), attributes (element 3), units (element 4), maximum value (element 5), minimum value (element 6), and data (element 7). Each element has a status, size, and value attribute.

SERCOS defines any controlled asynchronous event initiated by the master to the slave as a procedure. A procedure in initiated by the master writing $0 \times 3$ to the slave for the procedure's data. The slave then responds with $0 \times 7$ in the procedure's status to indicate the procedure is executing. When the procedure is completed, the slave changes the status from $0 \times 7$ to $0 \times 3$. The master then cancels the procedure by changing the data from $0 \times 3$ to $0 \times 0$. If the master wishes to interrupt the procedure at any time, it can write a $0 \times 1$ to the slave at which time the slave pauses the procedure and responds with a status of $0 \times 5$. The master can then either resume or cancel the procedure by writing $0 \times 3$ or $0 \times 0$ to the slave. If the procedure errors, the slave will stop the procedure and change the procedure's status from $0 \times 7$ to $0 x F$. The master must then cancel the procedure before it can be restarted. More than one procedure can be active on the slave at any one time.

SERCOS defines the process for initializing the ring in 5 phases. In Communication Phase 0 (CP0), the master sends out a sync pulse on the ring until it receives the pulse back meaning all drives are operating in repeat mode. CP0 is the only lower phase from which higher phase may be switched. As soon as the sync pulse is received, the master transitions the ring to Communication Phase 1 (CP1). In CP1, the master queries all the drive addresses that it wishes to initialize during that session. As soon as the master has found all desired drives on the ring and no errors have occurred, the ring transitions to Communication Phase 2 (CP2). During CP2, the master queries each of the drives for their timing parameters, then it calculates the necessary 
parameters for synchronization including each drive's communication time slot and downloads these parameters to each drive. In addition to the timing parameters, the master also sets up the control modes and cyclical channel data for each of the drives in this phase. As soon as the master has completed setting up each of the drive's timing parameters, the master starts the procedure 'Communication Phase 3 Transition Check' defined by IDN 127. As soon as all drives successfully complete this procedure, the master takes the ring to Communication Phase 3 (CP3). From CP3 on up, the all communications are done synchronously. During CP3, all remaining parameters necessary for control in each of the drives are set up. The master initiates transition from CP3 to Communication Phase 4 (CP4) by starting the procedure 'Communications Phase 4 Transition Check' defined by IDN 128. When all drives have properly executed the procedure, the master takes the ring to CP4. Upon switching to CP4, the initialization of the ring is complete.

In CP4, the bits 13-15 in the drive control and status words become active and control the starting and stopping of the drive. Prior to CP4, SERCOS specifies that the main power shall not be applied to the drive. As soon as the drive gets to $\mathrm{CP} 4$, it performs all computations for setting up the session. It keeps Bits 14 and 15 in the status word low during this period indicating that it is not ready for drive power. As soon as the drive has set up its drive logic, Bit 14 in the status word is set high indicating that the drive is ready for power. At this juncture, the main power may be applied to the drive. As soon as the main bus comes up to an acceptable level, the drive sets bit 15 high and bit 14 low thereby indicating that it is ready to operate. At this point, the master may enable the drive by setting bits 14 and 15 in the control word high. When the master enables the drive, the drive responds to the master by setting bit 14 high indicating that it is enabled. The drive then follows the commands from the control unit. The shutdown procedure is reverse of the startup procedure with the master disabling the drive (Control Bits 14,15= Low), the drive then slows the motor to zero velocity in a safe manner and removes power from the motor (Status Bit $15=$ High, Bit $14=$ Low), then main power may be removed from the drive and the drive remains in drive logic ready state (Status Bit $15=$ Low, Bit $14=$ High). If an error should occur in the drive, bit 13 in the status word is set high and the error is recorded in IDN 11, the error number in IDN 129, and error message in IDN 95, and the drive is disabled in a safe fashion if it is enabled. As soon as the drive has stopped and disabled, the drive waits for a Reset Class 1 diagnostic procedure defined by IDN 99. As soon as IDN 99 procedure is started, the drive tries to clear the fault. If the fault is successfully cleared, the drive then sets the status bits 13-15 low and the drive returns to Drive Logic Ready state and proceeds through the startup process previously described.

When $24 \mathrm{~V}$ power (the control voltage) is applied to the DISC ${ }^{\mathrm{TM}}$, it will come out of a power on reset and boot the controller. This usually takes 1 to 8 seconds after power has been applied. As soon as the processor comes out of reset, the state machine checks the resident memory and then sets up the internal CPU registers. If no errors occur during these tasks, the state machine will move on to DISC ${ }^{\mathrm{TM}}$ Initialization. If there is a system error at this juncture, it will be treated as a fatal error and send the processor into an eternal loop until a watchdog reset occurs. After the reset, the state machine will start the initialization process over again.

In the DISC ${ }^{\mathrm{TM}}$ initialization state, the DISC ${ }^{\mathrm{TM}}$ is initialized, the internal variables are aligned with the SERCOS IDNs, and the SERCOS ring is brought up to Phase4Work state, an internal sub-state of CP4. The DISC ${ }^{\mathrm{TM}}$ automatically synchronizes up to the bus speed dictated by the bus master during CP0. If the master in CP1 does not address the DISC ${ }^{\mathrm{TM}}$, then the 


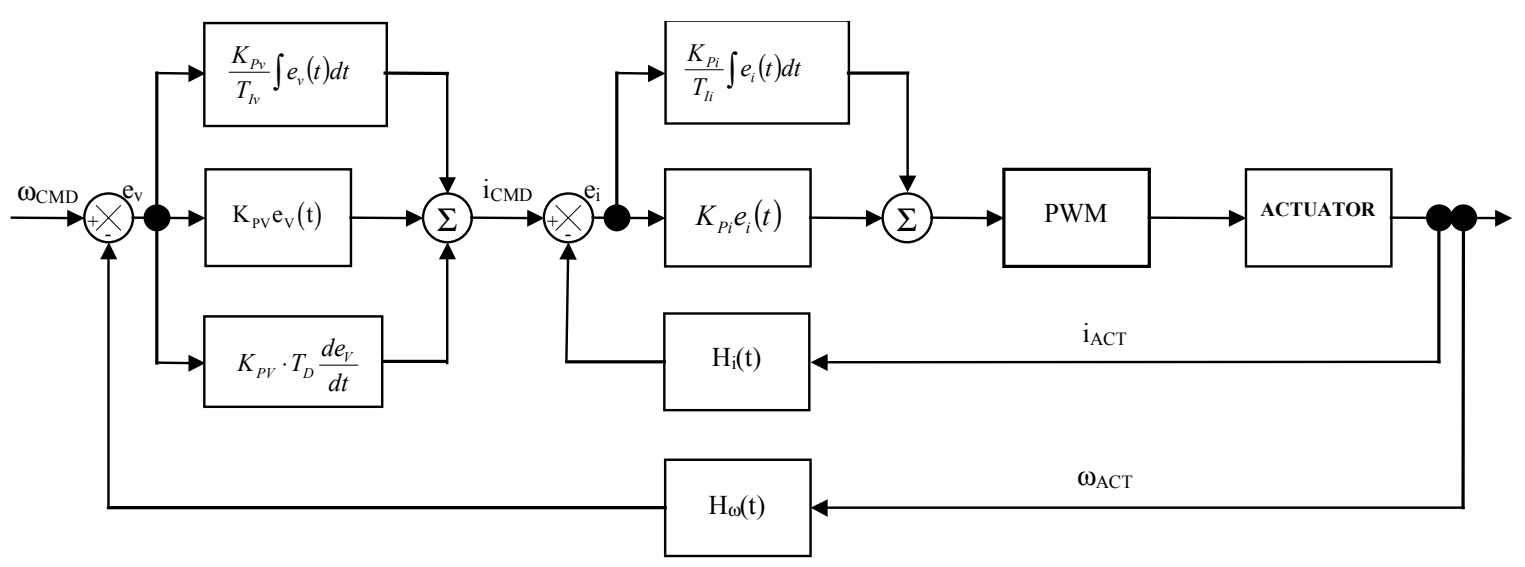

Figure 21: DISC ${ }^{\mathrm{TM}}$ PID control diagram.

DISC $^{\text {TM }}$ will remain in the DISC ${ }^{\text {TM }}$ Initialization state during the current session monitoring the ring for a new CP0. If an error occurs during the initialization of the $\mathrm{DISC}^{\mathrm{TM}}$, the drive records the error in IDN 129 and waits for further instruction from the master. If the error is a communication's error, the drive resets the SERCOS controller and waits for another CP0.

The DISC ${ }^{\text {TM }}$ transitions to the Drive Logic Ready state as soon as it has initialized all internal variables and its logic and the communication's state has transitioned to Phase4Work. The drive stays in this state until either the main power is enabled, an error occurs, or the communication's state changes from Phase4Work. As soon as the main power is turned on, the

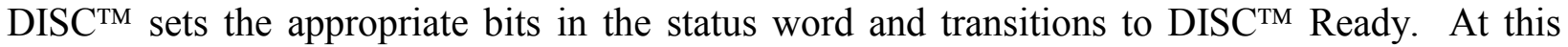
point, the sensor variables (current, temperature, voltage, etc) become active and their values can be queried over the service channel.

The DISC ${ }^{\text {TM }}$ Ready state is where the actuator remains until it is enabled. If the DISC ${ }^{\text {TM }}$ is attached to an actuator, then it cannot transition out of this state until the drive controlled homing procedure (IDN 148) has been initiated. For the homing procedure that is required by the DISCActuator ${ }^{\mathrm{TM}}$, the DISC ${ }^{\mathrm{TM}}$ homes the actuator, sets its new position to the homed value and proceeds to the DISC ${ }^{\mathrm{TM}}$ Enabled state provided no error occurred during the homing procedure. If main power is disabled, the DISC ${ }^{\text {TM }}$ will transition back to DISC ${ }^{\text {TM }}$ Logic Ready State. If the DISCActuator ${ }^{\mathrm{TM}}$ needs to be re-calibrated, the calibration procedure will need to be initiated from this state.

The DISC ${ }^{\text {TM }}$ transitions to the DISC ${ }^{\text {TM }}$ Enabled state when bits 14 and 15 in the control word are set high. In this state, the DISC ${ }^{\mathrm{TM}}$ controls the motor in either torque or velocity mode which is set during CP2 via IDN 32. The control for the DISC ${ }^{\mathrm{TM}}$ is traditional PID which is shown in Figure 21. SERCOS defines the integral action time $\left(\mathrm{T}_{\mathrm{i}}\right)$ and the differential time $\left(\mathrm{T}_{\mathrm{d}}\right)$ and then their respective gains are derived by the multiplication of $\mathrm{K}_{\mathrm{p}}$. Thus, a decrease in $\mathrm{T}_{\mathrm{i}}$ (IDN 101 and IDN 107 for the velocity and current loops, respectively) increases the overall integral gain. The control normalizes the plant model in order to maintain compatibility across many plants. As a result, the best proportional gains are chosen to be between 1 and 6 . The current is sampled at 4 times the closed loop rate. The position is sampled at the closed loop rate and the velocity is differentiated from the position. Future updates to the DISC ${ }^{\text {TM }}$ will separate out the closed loop rates, which are now optimized to minimize velocity noise from the differentiation of the position. The system PWM frequency is $31.25 \mathrm{kHz}$. 


\begin{tabular}{|c|c|c|c|c|c|}
\hline Error \# & Description & F/R & Error \# & Description & F/R \\
\hline \multicolumn{3}{|c|}{ Internal Errors } & \multicolumn{3}{|c|}{ Homing and Calibration Errors } \\
\hline $0 \times 0010$ & Unexpected Communication Error & $\mathrm{F}$ & $0 \times 4500$ & Homing Error & $\mathrm{R} 4$ \\
\hline $0 \times 0011$ & Out of Memory & $\mathrm{F}$ & $0 \times 4501$ & Error Initing Abs. Pos. Calibration Proc. & R4 \\
\hline $0 \times 0012$ & Invalid Argument & $\mathrm{F}$ & $0 \times 4502$ & Error During Abs. Pos. Calibration Proc. & R4 \\
\hline $0 \times 0013$ & Invalid Pointer & $\mathrm{F}$ & $0 \times 4503$ & Error Closing Abs. Pos. Calibration Proc. & R4 \\
\hline $0 \times 0014$ & Cannot Initialize Machine State Register & $\mathrm{F}$ & $0 \times 4504$ & AtoD Overrun Error & $\mathrm{F}$ \\
\hline $0 \times 0015$ & Cannot Initialize System Interrupt Space & $\mathrm{F}$ & $0 \times 4505$ & Error Commutating Transistors & R4 \\
\hline $0 \times 0016$ & Error Setting Up Supervisor Space & $\mathrm{F}$ & \multicolumn{3}{|c|}{ Manufacturer Specific Errors } \\
\hline $0 \times 0017$ & Error Setting Up Internal Bus Space & $\mathrm{F}$ & $0 \times 8000$ & Abs. Position Sensor Value Out of Range & R4 \\
\hline $0 \times 0018$ & Error Setting Up Internal Bus Control & $\mathrm{F}$ & $0 \times 8001$ & Current RMS Table Not Initialized & R0 \\
\hline $0 \times 0019$ & Error Setting Up Internal Timer \#1 & $\mathrm{F}$ & $0 \times 8002$ & Undefined PSI Board & $\mathrm{F}$ \\
\hline $0 \times 001 \mathrm{~A}$ & Error Setting Up Internal Timer \#2 & $\mathrm{F}$ & $0 \times 8003$ & Invalid Torque Direction & R0 \\
\hline $0 \times 001 \mathrm{~B}$ & Error Initializing Special Register 0 & $\mathrm{~F}$ & $0 \times 8004$ & Improper Torque Scaling & R0 \\
\hline $0 \times 001 \mathrm{C}$ & Error Initializing Special Register 1 & $\mathrm{~F}$ & $0 \times 8005$ & PWM System Not Counting & $\mathrm{F}$ \\
\hline $0 \times 001 \mathrm{D}$ & Error Initializing Special Register 2 & $\mathrm{~F}$ & $0 \times 8006$ & Error Initializing PWM Counter & R0 \\
\hline $0 \times 001 \mathrm{E}$ & Error Initializing Special Register 3 & $\mathrm{~F}$ & $0 \times 8007$ & PWM Prescale Out of Range & R0 \\
\hline $0 \times 001 \mathrm{~F}$ & Error Initializing Internal Memory Map & $\mathrm{F}$ & $0 \times 8008$ & PWM Scale Factor Out of Range & R0 \\
\hline $0 \times 0020$ & Error Initializing Decrementer & $\mathrm{F}$ & \multicolumn{3}{|c|}{ Analog To Digital Errors } \\
\hline $0 \times 0021$ & Error Initializing Motor Halls & Ro & 0xF030 & AtoD Clock Too Fast & $\mathrm{F}$ \\
\hline $0 \times 0022$ & Error Initializing Commutation System & R0 & $0 x F 031$ & AtoD Clock Too Slow & $\mathrm{F}$ \\
\hline $0 \times 0023$ & Encoder Error \# 1 & $\mathrm{R} 4$ & $0 x F 032$ & AtoD Pin Defined as Digital I/O & $\mathrm{F}$ \\
\hline $0 \times 0024$ & Encoder Error \# 2 & $\mathrm{R} 4$ & \multicolumn{3}{|c|}{ Serial Communications Interface Errors } \\
\hline $0 \times 0025$ & Encoder Error \# 3 & R4 & 0xF040 & Undefined Serial Word Size & R0 \\
\hline \multicolumn{3}{|c|}{ SERCOS Errors } & 0xF041 & Word Size Too Big & R0 \\
\hline $0 \times 0050$ & Invalid Memory Offset & $\mathrm{F}$ & 0xF042 & Baud Rate Too Slow & R0 \\
\hline $0 \times 0051$ & Telegram Type Not Supported & R0 & 0xF043 & Baud Rate Too Fast & R0 \\
\hline $0 \times 0052$ & MST Missing Twice & R0 & 0xF044 & Handshake Delay Too Big & R0 \\
\hline $0 \times 0053$ & Master Enable Out of Sequence & $\mathrm{R} 4$ & 0xF045 & Invalid Queue Wrap Address & R0 \\
\hline $0 \times 0054$ & Slave Memory Not Initialized & $\mathrm{F}$ & 0xF046 & Data Queue Too Large & R0 \\
\hline $0 \times 0055$ & Improper Cyclical Data & R0 & \multicolumn{3}{|c|}{ Data Initializations Errors } \\
\hline \multicolumn{3}{|c|}{ Interrupt Errors } & 0xF060 & Error Multiplexing Interrupts & $\mathrm{F}$ \\
\hline $0 \times 3000$ & SERCOS System Reset & $\mathrm{F}$ & 0xF061 & Invalid SRAM Voltage Error & $\mathrm{F}$ \\
\hline $0 \times 3001$ & Non Recoverable Machine Check & $\mathrm{F}$ & & Data Bus Error & \\
\hline $0 \times 300$ & Non Recoverable Floating Point Error & $\mathrm{F}$ & 0xF070 & Invalid Data Bus Address & $\mathrm{F}$ \\
\hline $0 \times 300$ & Non Recoverable Decrementer Error & $\mathrm{F}$ & \multicolumn{3}{|c|}{ Parallel I/O Port Error } \\
\hline $0 \times 300$ & Undefined Internal Exception & $\mathrm{F}$ & 0xF078 & Cannot Write to an Input Pin & $\mathrm{F}$ \\
\hline $0 \times 300$ & Undefined External Exception & $\mathrm{F}$ & \multicolumn{3}{|c|}{ Internal Counter Errors } \\
\hline & SERCOS Defined DISC'M Errors & & 0xF080 & Invalid Counter Mode & $\mathrm{F}$ \\
\hline $0 \times 4000$ & Heat Sink Overtemperature & $\mathrm{R} 4$ & 0xF081 & Reserved Mode & $\mathrm{F}$ \\
\hline $0 \times 4001$ & DISCTM Overtemperature & R4 & 0xF082 & No Clock Selected & $\mathrm{F}$ \\
\hline $0 \times 4002$ & Motor Overtemperature & $\mathrm{R} 4$ & \multicolumn{3}{|c|}{ Sine Commutation Errors } \\
\hline $0 \times 4003$ & Torque Overload & R4 & 0xF200 & Elect. Commutation Angle Out of Range & R0 \\
\hline $0 \times 4004$ & Velocity Limit & $\mathrm{R} 4$ & 0xF201 & PWM Command Out of Range & R0 \\
\hline $0 \times 4005$ & Position Limit & $\mathrm{R} 4$ & 0xF202 & PWM System Not Reset Properly & R0 \\
\hline $0 \times 4006$ & RMS Current Limit Exceeded & $\mathrm{R} 4$ & 0xF203 & PWM System Not Set Properly & Ro \\
\hline $0 \times 4007$ & Peak Current Limit Exceeded & R4 & \multicolumn{3}{|c|}{ Temperature Sensor Error } \\
\hline $0 \times 4008$ & Main Bus Undervoltage Error & R4 & $0 x F 250$ & Temperature Sensor Is Asleep & $\mathrm{F}$ \\
\hline $0 \times 4009$ & Main Bus Overvoltage Error & R4 & \multicolumn{3}{|c|}{ Serial ROM Errors } \\
\hline $0 \times 400 \mathrm{~A}$ & Invalid Control Mode & R4 & $0 x F 400$ & ROM is Locked & $\mathrm{F}$ \\
\hline $0 \times 400 \mathrm{~B}$ & Position Limit & R4 & 0xF401 & ROM is Write Protected & $\mathrm{F}$ \\
\hline $0 \times 400 \mathrm{C}$ & Invalid Commutation State & $\mathrm{R} 4$ & 0xF402 & ROM Capacity Exceeded & $F$ \\
\hline
\end{tabular}

Table 12: DISC ${ }^{\mathrm{TM}}$ error numbers (in hexadecimal) associated with IDN 129.

If an error that is not fatal occurs in any state, the DISC ${ }^{\mathrm{TM}}$ will store the error number in IDN 129 and set the appropriate bit in IDN 11 and transition to DISC ${ }^{\mathrm{TM}}$ error state. Note that IDN 11 can record multiple errors whereas IDN 129 and 95 can only record the most recent error. As soon as it gets to the DISC ${ }^{\mathrm{TM}}$ error state, the DISC ${ }^{\mathrm{TM}}$ decelerates the drive to zero velocity and then disables the power electronics. Then if the error is not a communication error, it will wait for a Reset Class 1 Diagnostics procedure to be initiated. If the error is a 


\begin{tabular}{|c|c|c|c|}
\hline IDN \# & Description & IDN \# & Description \\
\hline 1 & Control Unit Cycle Time (TNcyc) & 52 & Reference Distance 1 \\
\hline 2 & Communication Cycle Time (TScyc) & 53 & Position Feedback Value 2 \\
\hline 3 & Shortest AT Transmission Time (T1min) & 55 & Position Polarity Parameter \\
\hline 4 & Transmit/Receive Transition Time (TATMT) & 57 & Position Window \\
\hline 5 & Minimum Feedback Processing Time (T5) & 76 & Position Data Scaling Type \\
\hline 6 & AT Transmission Starting Time (T1) & 79 & Rotational Position Resolution \\
\hline 7 & Feedback Acquisition Point (T4) & 80 & Torque Command Value \\
\hline 8 & Command Value Valid Time (T3) & 82 & Positive Torque Limit Value \\
\hline 9 & Position of Data Record in MDT & 83 & Negative Torque Limit Value \\
\hline 10 & Master Data Telegram Length & 84 & Torque Feedback Value \\
\hline 11 & Class 1 Diagnostic (C1D) & 85 & Torque Polarity Parameter \\
\hline 12 & Class 2 Diagnostic (C2D) & 86 & Torque/Force Data Scaling Type \\
\hline 13 & Class 3 Diagnostic (C3D) & 87 & Transmit To Transmit Recovery Time (TATAT) \\
\hline 14 & Interface Status & 88 & Receive To Receive Recovery Time (TMTSY) \\
\hline 15 & Telegram Type Parameters & 89 & MDT Transmission Starting Time (T2) \\
\hline 16 & Configuration List of the AT (Drive Telegram) & 90 & Command Value Proceeding Time (TMTSG) \\
\hline 17 & IDN List of All Operational Data & 91 & Bipolar Velocity Limit Value \\
\hline 18 & IDN List of Operation Data for CP2 & 92 & Bipolar Torque Limit Value \\
\hline 19 & IDN List of Operation Data for CP3 & 93 & Torque/Force Data Scaling Factor \\
\hline 21 & IDN List of Invalid Operation Data for CP2 & 94 & Torque/Force Data Scaling Exponent \\
\hline 22 & IDN List of Invalid Operation Data for CP3 & 95 & Diagnostic Message \\
\hline 24 & Configuration List of the Master Data Telegram & 96 & Slave Arrangement (SLKN) \\
\hline 25 & IDN List of All Procedure Commands & 97 & Mask Class 2 Diagnostic \\
\hline 28 & MST Error Counter & 98 & Mask Class 3 Diagnostic \\
\hline 29 & MDT Error Counter & 99 & Reset Class 1 Diagnostic \\
\hline 30 & Manufacturer's Version & 100 & Velocity Loop Proportional Gain \\
\hline 32 & Primary Operation Mode & 101 & Velocity Loop Integral Action Time \\
\hline 33 & Secondary Operation Mode 1 & 102 & Velocity Loop Differential Time \\
\hline 34 & Secondary Operation Mode 2 & 104 & Position Loop Proportional Gain \\
\hline 35 & Secondary Operation Mode 3 & 105 & Position Loop Integral Action Time \\
\hline 36 & Velocity Command Value & 106 & Current Loop Proportional Gain 1 \\
\hline 37 & Additive Velocity Command Value & 107 & Current Loop Integral Action Time 1 \\
\hline 38 & Positive Velocity Limit Value & 109 & Motor Peak Current \\
\hline 39 & Negative Velocity Limit Value & 110 & Amplifier Peak Current \\
\hline 40 & Velocity Feedback Value & 111 & Motor Continuous Stall Current \\
\hline 41 & Homing Velocity & 112 & Amplifier Rated Current \\
\hline 42 & Homing Acceleration & 113 & Maximum Motor Speed \\
\hline 43 & Velocity Polarity Parameter & 114 & Load Limit of the Motor \\
\hline 44 & Velocity Data Scaling Type & 116 & Resolution of Feedback 1 \\
\hline 45 & Velocity Data Scaling Factor & 121 & Input Revolutions of Load Gear \\
\hline 46 & Velocity Data Scaling Exponent & 122 & Output Revolutions of Load Gear \\
\hline 47 & Position Command Value & 124 & Standstill Window \\
\hline 49 & Positive Position Limit Value & 125 & Velocity Threshold (nx) \\
\hline 50 & Negative Position Limit Value & 126 & Torque Threshold (Tx) \\
\hline 51 & Position Feedback Value 1 & 127 & Communication Phase 3 Transition Check \\
\hline
\end{tabular}

Table 13: SERCOS IDNs implemented on the DISC ${ }^{\text {TM }}$.

communication error, the DISC ${ }^{\mathrm{TM}}$ will immediately proceed to the DISC ${ }^{\mathrm{TM}}$ Initialization state and wait for the ring to be reset to $\mathrm{CP} 0$.

A listing of all errors handled by the DISC ${ }^{\mathrm{TM}}$ is given in Table 12. The F/R column represents whether or not the error is fatal and if it is not fatal, the communication's phase that the error is recoverable. For instance, if the current RMS table is not initialized properly (error code 0x8001), then the communications must return to $\mathrm{CP} 0$ so the DISC ${ }^{\mathrm{TM}}$ can be reinitialized. After an error has been cleared and if the communication state is in Phase4Work, the DISC ${ }^{\text {TM }}$ will transition to the DISC ${ }^{\mathrm{TM}}$ Logic Ready state where it will re-initiate the start up procedure. If a fatal error occurs in the DISC ${ }^{\mathrm{TM}}$, the state machine records the fatal error. Then, it disables the power electronics and jumps to an eternal loop where it sets the watchdog timer count and waits for a reset to occur. The watchdog timer is set such that will reset the machine within $200 \mathrm{~ms}$. 


\begin{tabular}{|c|c|c|c|}
\hline IDN \# & Description & IDN \# & Description \\
\hline 128 & Communication Phase 4 Transition Check & 307 & Allocation of Real-Time Status Bit 2 \\
\hline 129 & Manufacturer Class 1 Diagnostic & 310 & Overload Warning \\
\hline 134 & Master Control Word & 311 & Amplifier Overtemperature Warning \\
\hline 135 & Drive Status Word & 312 & Motor Overtemperature Warning \\
\hline 138 & Bipolar Acceleration Limit & 313 & Cooling Error Warning \\
\hline 142 & Application Type & 330 & Status: $\mathrm{n}$ feedback $=\mathrm{n}$ command \\
\hline 143 & SERCOS Interface Version V01.02 & 331 & Status: $\mathrm{n}$ feedback $=0$ \\
\hline 146 & Control Unit Controlled Homing Procedure Cmd & 332 & Status: $\mathrm{n}$ feedback $<\mathrm{nx}$ \\
\hline 147 & Homing Parameter & 333 & Status: $T>=T x$ \\
\hline 148 & Drive Controlled Homing Procedure Command & 334 & Status: T > TLimit \\
\hline 150 & Reference Offset 1 & 335 & Status: $\mathrm{n}$ command > nLimit \\
\hline 157 & Velocity Window & 336 & Status: In Position \\
\hline 158 & Power Threshold (Px) & 337 & Status: $P>=P x$ \\
\hline 159 & Monitoring Window & 380 & DC Bus Voltage \\
\hline 173 & Marker Position A & 382 & DC Bus Power $(\mathrm{P})$ \\
\hline 181 & Manufacturer Class 2 Diagnostic & 383 & Motor Temperature \\
\hline 182 & Manufacturer Class 3 Diagnostic & 384 & Amplifier Temperature \\
\hline 185 & Length of Configurable Data in the AT & 407 & Homing Enable \\
\hline 186 & Length of Configurable Data in the MDT & 408 & Reference Mark Pulse Registered \\
\hline 187 & IDN List of Configurable Data in the AT & 32790 & Number of Motor Poles \\
\hline 188 & IDN List of Configurable Data in the MDT & 32804 & Motor Encoder Offset \\
\hline 189 & Following Distance & 32820 & Motor Hall States \\
\hline 191 & Cancel Reference Point Procedure Command & 32823 & Motor Hall Configuration Parameters \\
\hline 192 & IDN list of Backup Operation Data & 32848 & Motor Winding Resistance \\
\hline 196 & Motor Rated Current & 32879 & Motor Torque Constant \\
\hline 200 & Amplifier Warning Temperature & 32880 & Amplifier Maximum Current to Date \\
\hline 201 & Motor Warning Temperature & 32881 & Amplifier Maximum RMS Current to Date \\
\hline 202 & Cooling Error Warning Temperature & 32968 & Brake Enable Procedure Command \\
\hline 203 & Amplifier Shutdown Temperature & 32969 & Brake Enable \\
\hline 204 & Motor Shutdown Temperature & 32970 & Brake Duty Cycle \\
\hline 205 & Cooling Error Shutdown Temperature & 33000 & IDN List of Drive Enabled Write Protected Data \\
\hline 208 & Scaling of Temperature Data & 33050 & Position Limit Warning Delta \\
\hline 263 & Load Working Memory Procedure Command & 33123 & System Up Time \\
\hline 264 & Save Working Memory Procedure Command & 33256 & DC Bus Over Voltage Level \\
\hline 288 & IDN List of Write Protected Data After CP2 & 33261 & Heat Sink Temperature \\
\hline 289 & IDN List of Write Protected Data After CP3 & 33268 & Calibration Procedure Command \\
\hline 296 & Velocity Feed Forward Gain & 33269 & Raw Sine Sensor Data \\
\hline 300 & Real-Time Control Bit 1 & 33270 & Raw Cosine Sensor Data \\
\hline 301 & Allocation of Real-Time Control Bit 1 & 33271 & Sample Data Points \\
\hline 302 & Real-Time Control Bit 2 & 33272 & Sine Coefficients For Data Correction \\
\hline 303 & Allocation of Real-Time Control Bit 2 & 33273 & Cosine Coefficients For Data Correction \\
\hline 304 & Real-Time Status Bit 1 & 33274 & Absolute Position Sensor Zero Offset \\
\hline 305 & Allocation of Real-Time Status Bit 1 & 33275 & Absolute Position Sensor Calibration Status \\
\hline 306 & Real-Time Status Bit 2 & & \\
\hline
\end{tabular}

Table 14: SERCOS IDNs implemented on the DISC ${ }^{\mathrm{TM}}$ (continued from Table 13).

The error number causing the fatal error can then be read from IDN 129 in Phase 2 before a 'Reset Class 1 Diagnostic' procedure has occurred.

An IDN is a 16-bit number referencing a parameter in the drive. SERCOS has classified the IDNs into two categories: SERCOS standard IDNs called S-IDNs and product specific IDNs called P-IDNs. A SERCOS IDN has its most significant bit set to a 1 to identify it as a P-IDN. Table 13 and Table 14 present the IDNs that are implemented on the DISC ${ }^{\mathrm{TM}}$. A description of these IDNs can be found in [ARM, 2001]. All of these IDNs are active except 37, 52, 57, 104, $105,146,150,173,191$, and 408 . The inactive IDNs are primarily related to position control and system controller referenced homing protocol. Both of these features will be added to the DISC $^{\text {TM }}$ at future dates. In addition to these IDNs, IDNs 33268-33275 (P-500 through P-507), which are used by ARM during assembly for calibrating the absolute position sensor relative to the input encoder, should be ignored during normal operation. 


\begin{tabular}{|c|l|c|l|}
\hline IDN \# & \multicolumn{1}{|c|}{ Description } & IDN\# & \multicolumn{1}{|c|}{ Description } \\
\hline 32 & Primary Operation Mode & 109 & Motor Peak Current \\
\hline 41 & Homing Velocity (Only if Homing is req'd) & 111 & Motor Continuous Stall Current \\
\hline 42 & Homing Acceleration (Only if Homing is req'd) & 113 & Maximum Motor Speed \\
\hline 49 & Positive Position Limit Value (req'd for actuators) & 114 & Load Limit of the Motor \\
\hline 50 & Negative Position Limit Value (req'd for actuators) & 116 & Resolution of Feedback 1 (IDN 51) \\
\hline 82 & Positive Torque Limit Value & 122 & Output Revolutions of Load Gear \\
\hline 83 & Negative Torque Limit Value & 138 & Bipolar Acceleration Limit \\
\hline 100 & Velocity Loop Proportional Gain & 32790 & Number of Motor Poles \\
\hline 101 & Velocity Loop Integral Action Time & 32823 & Motor Hall Configuration Parameters \\
\hline 102 & Velocity Loop Differential Time & 32848 & Motor Winding Resistance \\
\hline 106 & Current Loop Proportional Gain & 32879 & Motor Torque Constant \\
\hline 107 & Current Loop Integral Action Time & 33050 & Position Limit Warning Delta (req'd for actuators) \\
\hline
\end{tabular}

Table 15: Minimum IDN setup list required for DISC ${ }^{\text {TM }}$ operation.

The programming of a SERCOS drive is done through the use of the IDN. For the DISC $^{\text {TM }}$ drives, they require the IDNs that are listed in Table 15, in addition to the SERCOS timing parameters, to be set for proper operation. If one is to change the scaling parameters from their default values for torque, velocity, or position, it is important that they are changed first in CP2 before any of their respective variables are set such as velocity limit. Note that the DISCActuators $^{\mathrm{TM}}$ automatically enable the position limits through IDN 55 even if they are not enabled during the initialization of the IDNs in CP2 and CP3. In order to assure that a variable is set up correctly, the DISC ${ }^{\mathrm{TM}}$ zeros any variable associated with the scaling parameter that is changed. It is suggested that the scaling parameters be changed in CP2 and the associated variables be set up in CP3.

\subsubsection{ARM32 Development}

The ARM32 development encompassed the development of the ARM32 mechanicals and the PSI32. While the CCI board is common to both the ARM20 and ARM32 modules, its development took place along with the ARM20 development and, thus, is described therein. The sub-sections below describe the development process for the ARM32. The actuator testing of the ARM32 is included with that of the ARM20 in Section 2.2.1.4.

\subsection{ARM32 Mechanical Development}

The ARM32 mechanicals include input and output bearings, input and output position sensors, input brake, gear train, brushless DC motor, wire coil, housing and miscellaneous machined components. Much of the design process and architecture of the ARM32 was similar to the ARM20 and will not be discussed herein.

The motor is a frameless custom wound motor with the design parameters specified in Table 16. The manufacturer incorporated Hall sensors for commutation and a $100 \Omega$ RTD sensor, for determining the winding temperature, into the motor's stator windings. The motor magnets were permanently mounted on a custom designed hollow rotor shaft. The hollow shaft allowed for the passage of the wire coil tube. Section 2.2.1.1.1 provides an explanation of Hall Configuration.

The gear train topology chosen for the actuator modules was the harmonic drive. The model chosen for the ARM32 was an off-the-shelf model designed and modified to incorporate the output bearing structure. The modification allowed ARM to reduce the cost and lead time of the module and still produce a significantly more compact actuator than what is on the market today. The ratings for the gear train are given in Table 17. 


\begin{tabular}{|l|c|c|}
\hline \multicolumn{1}{|c|}{ Parameter } & Value & Units \\
\hline \hline Rotor Inertia & $1.29 \times 10^{-4}$ & $\mathrm{~kg} \cdot \mathrm{m}^{2}$ \\
\hline Temperature Rise Per Watt & 1.3 & \\
\hline Detent Torque & 0.0177 & $\mathrm{~N} \cdot \mathrm{m}$ \\
\hline Torque Constant & 0.644 & $\mathrm{~N} \cdot \mathrm{m} / \mathrm{A}$ \\
\hline Voltage Constant & 0.644 & $\mathrm{~V} / \mathrm{rad} / \mathrm{sec}$ \\
\hline Winding Resistance & 6.37 & $\Omega$ \\
\hline Terminal Inductance & 10.8 & $\mathrm{mH}$ \\
\hline Continuous Rated Torque & 1.96 & $\mathrm{~N} \cdot \mathrm{m}$ \\
\hline No Load Speed & 367 & $\mathrm{rad} / \mathrm{sec}$ \\
\hline Peak Torque & 5.92 & $\mathrm{~N} \cdot \mathrm{m}$ \\
\hline Peak Current & 9.2 & $\mathrm{~A}$ \\
\hline Designed Voltage & 240 & $\mathrm{Volts}$ \\
\hline Number of Poles & 8 & Poles \\
\hline Hall (A-B-C) Configuration & $0 \times 312$ & - \\
\hline Winding Configuration & Delta & - \\
\hline
\end{tabular}

Table 16: ARM32 motor specifications.

The ARM32 input section and the PSI32 housings were obtained from the same extrusion. The housings were machined to accommodate pass through pneumatics connections, mounting holes for the PSI32 shell, input electrical connector, output housing interface and end plate. The housing also contains fins to aid in the dissipation of heat from the motor and PSI32 power electronics. The thermal dissipation constant $\left(\mathrm{R}_{\theta}\right)$ for the shell to ambient is estimated to be approximately 1.3 Watts $/{ }^{\circ} \mathrm{C}$. This value is comparable to motors of similar size and characteristics.

The bearings and seals for the ARM32 were designed and manufactured to obtain the maximum compactness to stiffness ratio possible. The input bearings, which were purchased off-the-shelf, have a total moment of inertia of $4 \times 10^{-5} \mathrm{~kg} \cdot \mathrm{m}^{2}$ and a limiting speed of 1361 $\mathrm{rad} / \mathrm{sec}$. The total drag torque for the input bearings is less than $0.015 \mathrm{~N} \cdot \mathrm{m}$. The output bearing housing is a proprietary compact custom-designed arrangement. Its drag torque reflected to the input was empirically found to be approximately $0.028 \mathrm{~N} \cdot \mathrm{m}$ and its mass moment of inertia

\begin{tabular}{|l|c|c|}
\hline \multicolumn{1}{|c|}{ Parameter } & Value & Units \\
\hline \hline Rotor Inertia & $1.60 \times 10^{-4}$ & $\mathrm{Kg} \cdot \mathrm{m}^{2}$ \\
\hline Gear Ratio & $100: 1$ & Cycles/Cycle \\
\hline Accuracy & 1.5 & $\mathrm{Arc} \cdot \mathrm{Min}$ \\
\hline Repeatability/Lost Motion & 40 & $\mathrm{Arc} \cdot \mathrm{Sec}$ \\
\hline No Load Starting Torque & 0.11 & $\mathrm{~N} \cdot \mathrm{m}$ \\
\hline Viscous Drag Torque & $8.0 \times 10-4$ & $\mathrm{~N} \cdot \mathrm{m} / \mathrm{rad} / \mathrm{sec})$ \\
\hline Continuous Rated Load Torque & 130 & $\mathrm{~N} \cdot \mathrm{m}$ \\
\hline Peak Load Torque & 298 & $\mathrm{~N} \cdot \mathrm{m}$ \\
\hline Rated Input Speed (Grease) & 367 & $\mathrm{Rad} / \mathrm{sec}$ \\
\hline Peak Input Speed (Grease) & 367 & $\mathrm{Rad} / \mathrm{sec}$ \\
\hline
\end{tabular}


reflected to the input is approximately $9 \times 10^{-7} \mathrm{~kg} \cdot \mathrm{m}^{2}$. The output bearing arrangement is designed to handle $23,800 \mathrm{~N}$ axial force, $10,700 \mathrm{~N}$ radial force and an overturning moment of $700 \mathrm{~N} \cdot \mathrm{m}$ for an L-10 life rating. Its maximum rated speed is $20.9 \mathrm{rad} / \mathrm{sec}$ or $200 \mathrm{RPM}$. The ARM32 housing incorporates many static seals and only two dynamic seals. The static seals are designed to keep the housing waterproof and maintain the pneumatic seal throughout the housing. The dynamic seals are located between the input and output housings. They are designed to perform two functions: seal the housing from outside particulates and maintain a pneumatic seal across the housing sections. In addition to the seals, a thermal barrier between the motor housing and the PSI32 shell was also designed and fabricated. The purpose of the thermal barrier is to thermally isolate the motor housing from the PSI32 housing.

The brake design was built on the design for the ARM 20. The braking material used for this device was the same cork that was used in the ARM20. The brake disk was pre-loaded such that it would break free if a load of approximately $8 \mathrm{~N} \cdot \mathrm{m}$ on the motor rotor $(\sim 80 \mathrm{~N} \cdot \mathrm{m}$ on the output) was applied to the motor rotor. The brake also included a switch to sense when the brake solenoid was disengaged from the disk. The switch allows a controller to reduce the power applied to the solenoid once it is disengaged from the disk. This results in a significant reduction in the power dissipated by the brake. There is a mechanical brake release built into the motor housing. It is operated by turning a $2.0 \mathrm{~mm}$ Allen wrench clockwise to disengage the solenoid from the disk. Furthermore, the release is designed to function as a pop-off valve in case the pneumatic pressure inside the actuator becomes too great.

For the ARM32 as with the ARM20, ARM chose to incorporate the highest resolution encoder wheel commercially available in input section. The resolution was 2568 counts before quadrature. This yields a resolution on the output of one count in 1,027,200 or about one arcseconds after quadrature, assuming a perfect gear train. The inertia of the encoder wheel is negligible compared to the rotor shaft. The wheel includes a $90^{\circ}$ index pulse and is at a custom diameter to accommodate the large through bore. The encoder head is specified to operate over a temperature range of $-40{ }^{\circ} \mathrm{C}$ to $100{ }^{\circ} \mathrm{C}$ with a maximum guaranteed count frequency of 100 $\mathrm{kHz}$. For the above resolution, this yields a maximum input shaft angular velocity of $245 \mathrm{rad} / \mathrm{sec}$ or 2340 RPM.

The output sensor is a proprietary sensor developed by ARM to coarsely sense the output position to within less than one turn of the input section over a range of $\pm 270^{\circ}$. This sensor is very similar to the design used for the ARM20. This sensor is calibrated using a proprietary Fast Fourier Transform (FFT) algorithm to increase the accuracy of the ARM32 sensor to less than 0.6 degrees and is aligned with the index pulse on the input sensor. This allows the DISC ${ }^{\text {TM }}$ to precisely know the output position by homing to the nearest index pulse, which can be found in less than $3.6^{\circ}$ for the ARM32.

The wire coil and connectors were designed as an integral component of the overall mechanical and electrical design of the ARM32 system much in the same manner as the ARM20. The ARM32 uses the same wire configuration as the ARM20 on both the proximal and distal sides. The current capacity of the connectors for the ARM32 is rated to handle a minimum of four ARM32 actuators or an excess of 14 amps for the power pins.

Figure 22 shows an ARM32 assembled actuator. Table 18 summarizes the final actuator design specifications and Figure 23 depicts the designed Torque-Speed curve. These specifications are based on a composite of the above specifications for each of the individual components. The continuous output torque is limited by both the continuous current rating of the 


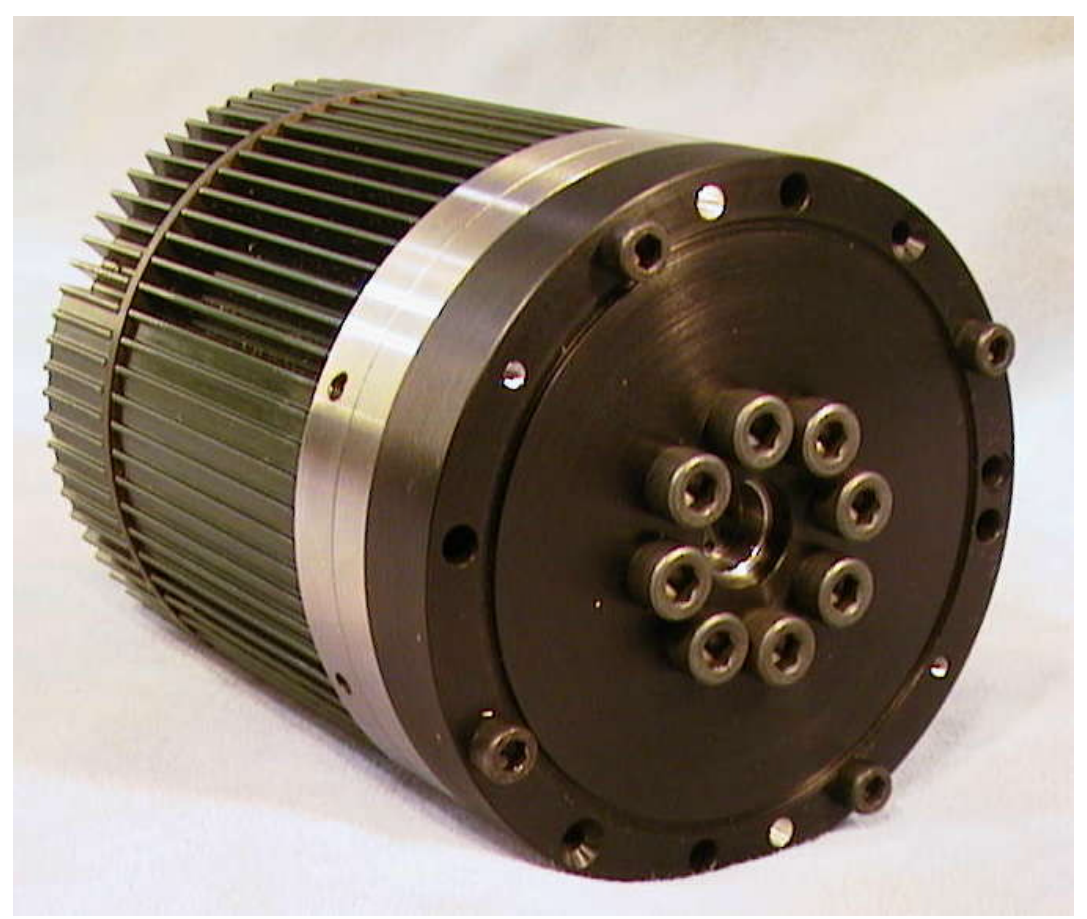

Figure 22: Assembled ARM32 actuator module.

PSI32 of 2.50 amps, and the continuous torque rating of the gear train. The peak torque rating of the gear train limits the actuator's peak torque rating. The peak speed of the actuator is limited by the encoder count frequency. The measured specifications are shown in Section 2.2.1.4.

\subsection{PSI32 Development}

The PSI32 was developed to control the ARM32 modular actuator. The PSI32 has four main design aspects: CCI and external interfaces, sensor interface logic, power interface logic, and power conversion and monitoring circuitry. The PSI32 was designed with the same environmental constraints as the CCI and the PSI20 boards.

The CCI and external interfaces consists of the CCI interface, the main power bus interface, the absolute position sensor interface, power output interface, motor sensor interface,

\begin{tabular}{|l|c|c|}
\hline & ARM32 (initial) & ARM32 (final) \\
\hline Performance: & & \\
\hline - Range of Motion & $\pm 340^{\circ}$ & $\pm 270^{\circ}$ \\
\hline - Peak Output Speed & $35 \mathrm{RPM}$ & $23.4 \mathrm{RPM}$ \\
\hline - Peak Output Torque & $333 \mathrm{~N} \cdot \mathrm{m}$ & $298 \mathrm{~N} \cdot \mathrm{m}$ \\
\hline - Continuous Torque & $137 \mathrm{~N} \cdot \mathrm{m}$ & $130 \mathrm{~N} \cdot \mathrm{m}$ \\
\hline - Bearing Load Capacity (axial/radial) & $7550 \mathrm{~N} / 3000 \mathrm{~N}$ & $23,800 \mathrm{~N} / 10,700 \mathrm{~N}$ \\
\hline - Bearing Overturning Load (L10 Life) & $755 \mathrm{~N} \cdot \mathrm{m}$ & $700 \mathrm{~N} \cdot \mathrm{m}$ \\
\hline Physical: & & $159 \mathrm{~mm}$ \\
\hline - Length & $160 \mathrm{~mm}$ & $129 \mathrm{~mm}$ \\
\hline - Diameter & $145 \mathrm{~mm}$ & $6.1 \mathrm{~kg}$ \\
\hline - Mass & $4.5 \mathrm{~kg}$ & \\
\hline
\end{tabular}




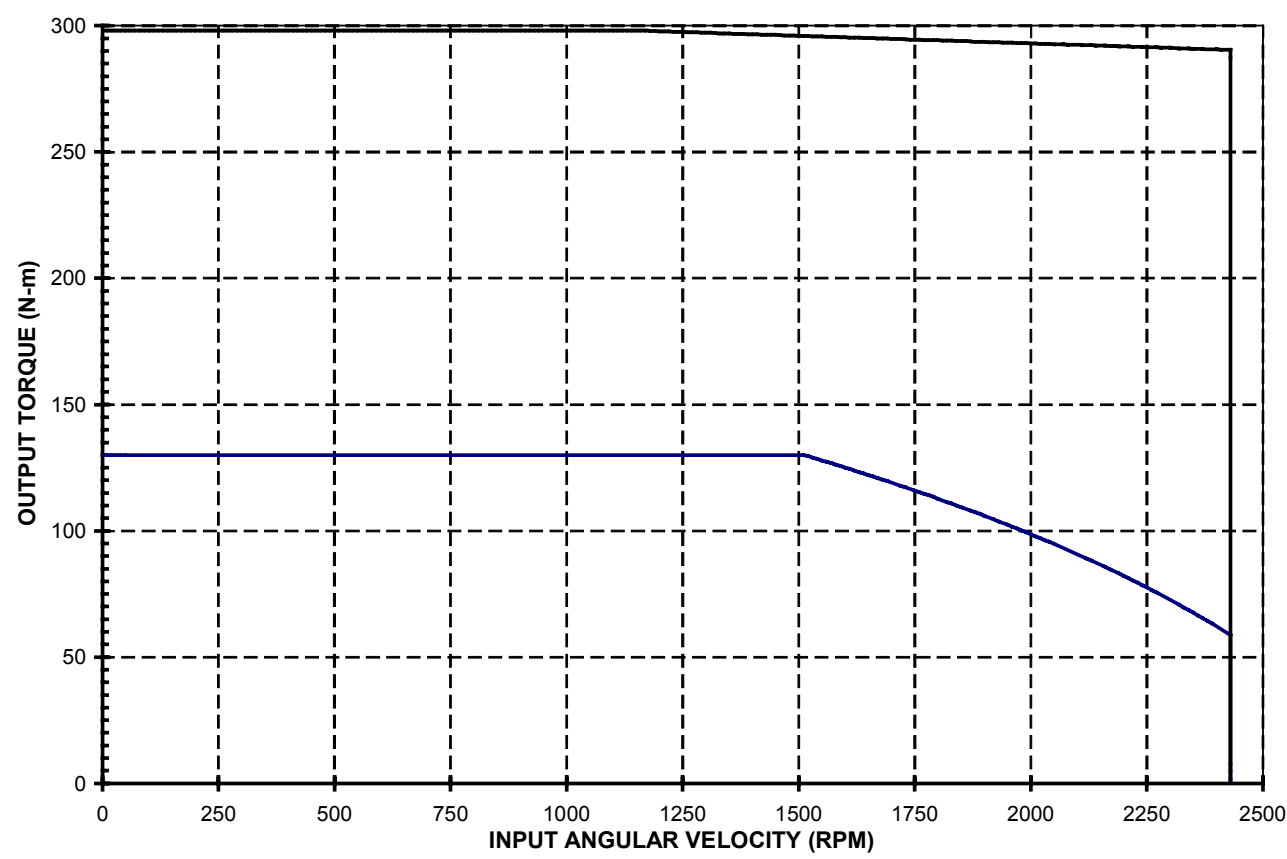

Figure 23: ARM32 designed torque-speed curve.

encoder and brake switch interface, and PSI32 thermistor interface (only one thermistor on the PSI32). The PSI to CCI interface contains the following signals: SERCOS data bus, 3 PWM channels, PWM output enable, 6 commutation lines, quadrature encoder and index signals, 3 Hall signals, commutation select signal, brake enable signal, 6 data lines, address line, overcurrent interrupt, 2 SPI channels, brake PWM signal, brake disengaged feedback signal, current filter clock, main power bus present signal, and 6 AtoD channels. The board connects to the main wire harness through a pigtail. The pigtail has the same 8 signals as the PSI20. The $24 \mathrm{~V}$ bus is fused by a 2 Amp fuse, and the main power bus is fused by a $7.5 \mathrm{~A}$ fuse. The $7.5 \mathrm{Amp}$ fuse is necessary to handle surges on the main power bus during startup and shutdown of the motor. The power output interface connects the PSI32 to the brushless DC motor, dynamic braking resistor, and the brake. The motor sensors are the Hall sensors and a RTD sensor integral to the stator winding.

The sensor interface logic conditions the sensor signals before they are sent to the CCI board. This logic incorporates the same sensor conditioning circuitry as the PSI20 except for the current and bus voltage sensor. Additionally, the PSI32 only uses one thermistor to sense the housing temperature. Any signal that is the same as the PSI20 will not be discussed herein (see Section 2.2.1.2 for more further information).

The current sense circuit is optimized for sensing the motor's current over the range of 0 to 5 amps. This provides maximum resolution over the motor's specified current range. Figure 24 shows the designed relationship between the sensed current and the output voltage (slope = 0.19V/Amp). Unlike the ARM20, this current senses the bipolar current for two of the motors windings and is electrically isolated from the high voltage bus. This was possible due to the availability of more volume. The output of each current sensor is filtered by a single pole low pass passive filter with a cutoff frequency of $4.8 \mathrm{kHz}$ and an 8-pole switched capacitor Bessel filter with a software selectable cutoff frequency. 


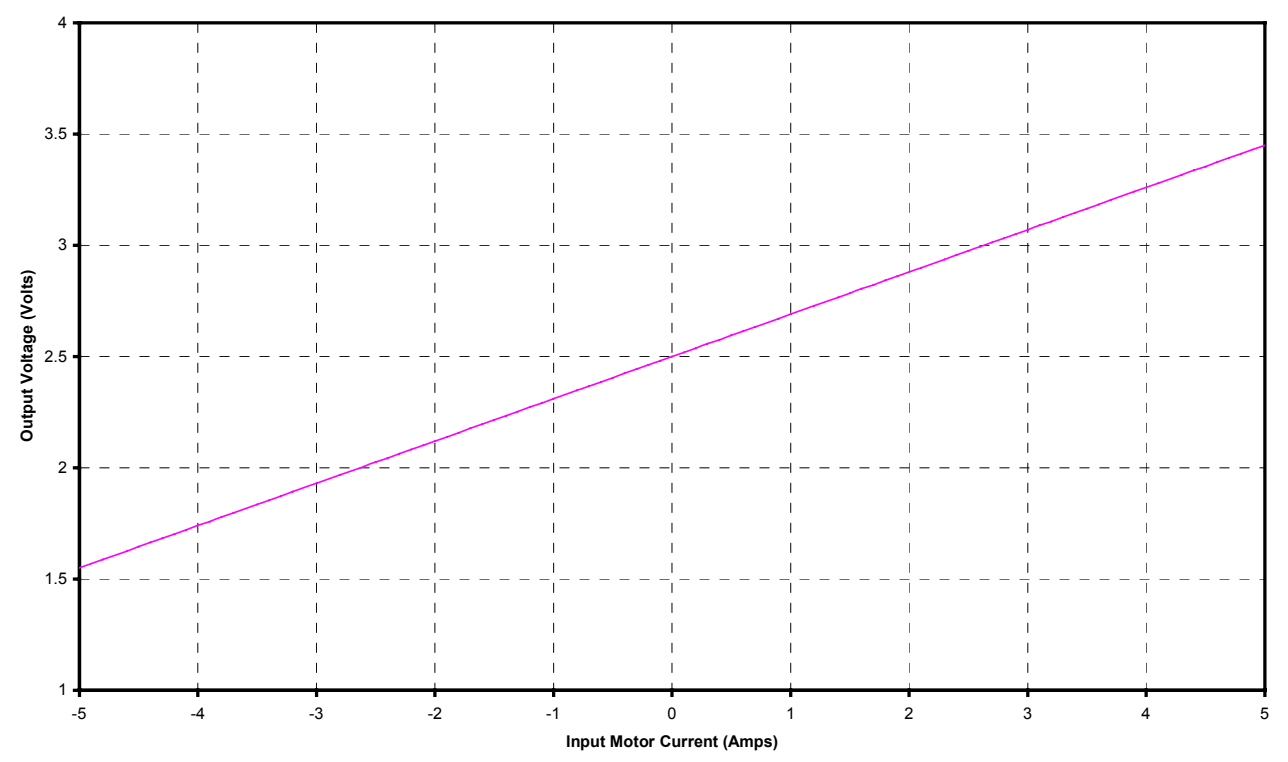

Figure 24: Designed input motor current versus output voltage curve.

The main bus voltage is sensed using an opto-coupler circuit operating its linear region with feedback to linearize the output. The feedback makes this circuit more reproducible since most of the sensor's non-linearities are removed. Figure 25 shows the relationship of the output voltage to the input voltage. The slope for the PSI32 is positive as opposed to PSI20's negative slope shown in Figure 16. Note that this circuit cannot sense the bus voltage very accurately until it is greater than about $20 \mathrm{~V}$. This circuit provides greater than 2500 volts isolation from the high voltage circuit while dissipating very little power (less than one watt). This output of the circuit is also used by the dynamic braking circuitry in the power interface logic.

The power interface logic contains the following functionality: PWM control, threephase motor transistor driver, motor transistors, and the brake drive. The PWM control functionality is carried out in a CPLD. It handles the functionality for the motor gate drive, the brake gate drive, and the board identity word. The motor gate drive generates the six output transistor drive states based on the input commutation topology, the three PWM states, the PWM enable and the six commutation states. The CPLD logic design allows the PSI32 to commutate the motor in either sinusoidal or trapezoidal modes. The brake gate drive allows full voltage to be supplied to the brake coil until the brake is disengaged. Once the brake is disengaged, the holding current is reduced from about 3 amps to about 0.6 amps, which decreased the power dissipated by a factor of approximately 20 . If the brake becomes engaged during operation due to a shock, full power is reapplied across the coil until it is again disengaged. The motor transistor drive circuitry receives the opto-isolated transistor drive signals from the PWM logic and level shifts them to the appropriate level for driving the gates of the transistors. This circuitry also assures that no two transistors on the same leg are turned on at the same time causing an instantaneous shoot through situation. This circuitry also monitors the current on the main power bus for an over-current situation. The circuit is designed to disable the power in approximately $2 \mu \mathrm{s}$. This circuit implements the corrections to the errors found in the testing of the PSI20s as discussed at the end of Section 2.2.1.1.3. The motor transistor circuitry is a threephase H-bridge configuration whose transistors are optimally designed to handle 2.50 Amps, continuous current, and 5.0 Amps, peak current and a bus voltage up to $320 \mathrm{~V}$. Gate drives for 


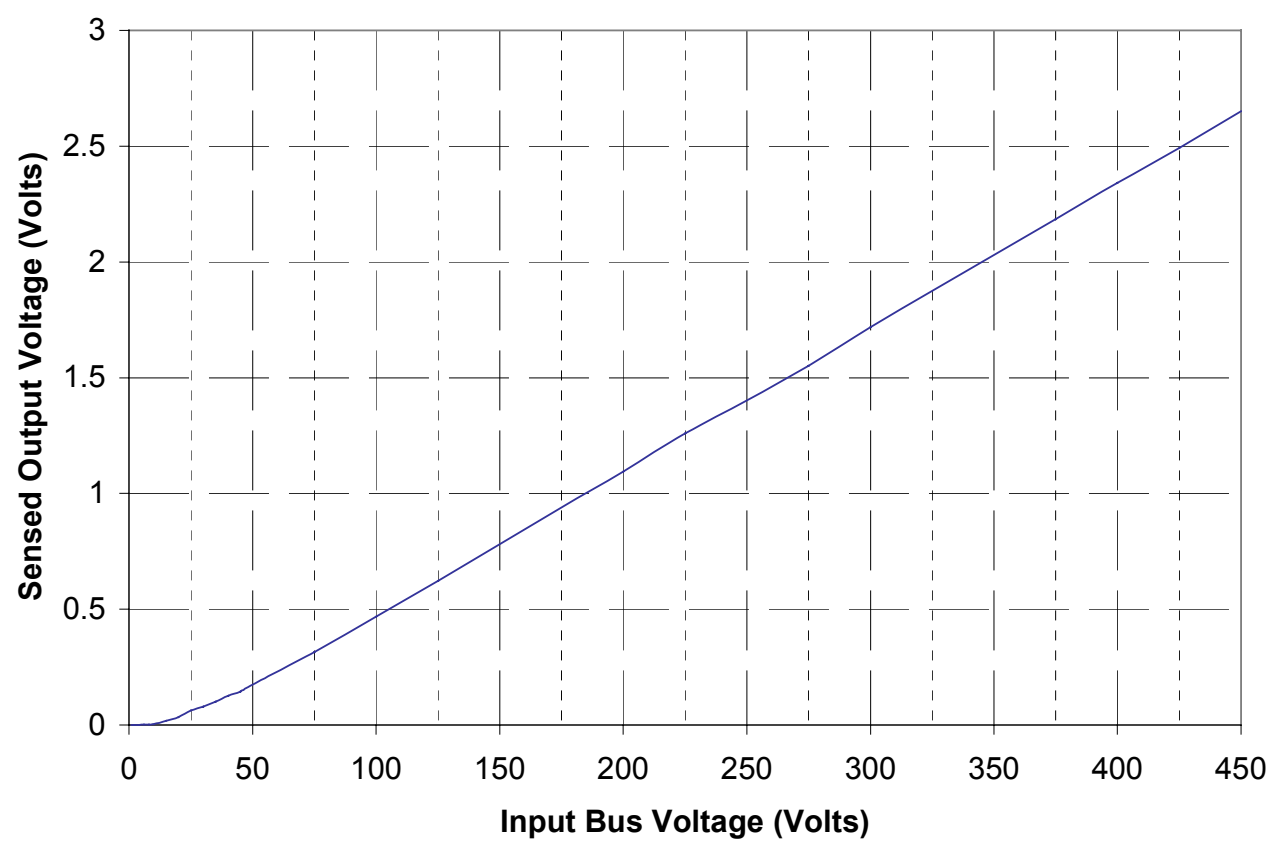

Figure 25: PSI32 empirical curve for input bus voltage versus output voltage.

the transistors implement the corrections to the original circuit found during the testing of the PSI20s. The brake drive circuitry is also optically isolated from the logic side of the PSI32.

The power conditioning circuitry generates the $5 \mathrm{~V}$ for the digital and analog circuitry, and the $15 \mathrm{~V}$ for the transistor gate drive circuitry, and dynamically bleeds the main power bus. The main bus bleed circuit is designed to dynamically brake the motor bus at $412 \mathrm{~V},+/-1 \mathrm{~V}$. This circuit has a hysteresis of approximately 45 volts.

Figure 26 shows a completed PSI32 mounted in the same extrusion that was used for the ARM32 motor housing. The final diameter of the board is $96 \mathrm{~mm}$. The board has 194 passive and active components and 165 nets. The board is laid out in 6 layers and fabricated with $2 \mathrm{oz}$. copper on polyimide material. The board also has 342 through-hole pads and vias. All components on the board are rated for a maximum ambient temperature of $85{ }^{\circ} \mathrm{C}$ with all but four of the components rated for a peak temperature of more than $105^{\circ} \mathrm{C}$. The $24 \mathrm{~V}$ bus draws 0.08 Amps of quiescent current for powering the external sensors and the PSI32 board when no CCI board, brake or motor is attached. Table 19 summarizes the PSI32 final specifications.

Unlike the PSI20, the PSI32 was not fully prototyped. Its circuit design was based on

\begin{tabular}{|l|c|c|}
\hline \multicolumn{1}{|c|}{ Parameter Specified } & Specification & Units \\
\hline \hline - Temperature Range (Min. for all comp.) & $-40<\mathrm{T}<85$ & ${ }^{\circ} \mathrm{C}$ \\
\hline - Life (MTBF) & $>15,000$ & Hrs. \\
\hline - Outside Diameter & 71 & $\mathrm{~mm}$ \\
\hline - Thickness excluding connectors & $12 \mathrm{~mm}$ & $\mathrm{~mm}$ \\
\hline - Operating Voltage Input & 24 & Volts \\
\hline - Total Power Consumption (quiescent) & $<2$ & Watts \\
\hline - Main Bus Voltage Input & $<310$ & Volts \\
\hline - Max. Motor Power Output & 250 & Watts \\
\hline
\end{tabular}




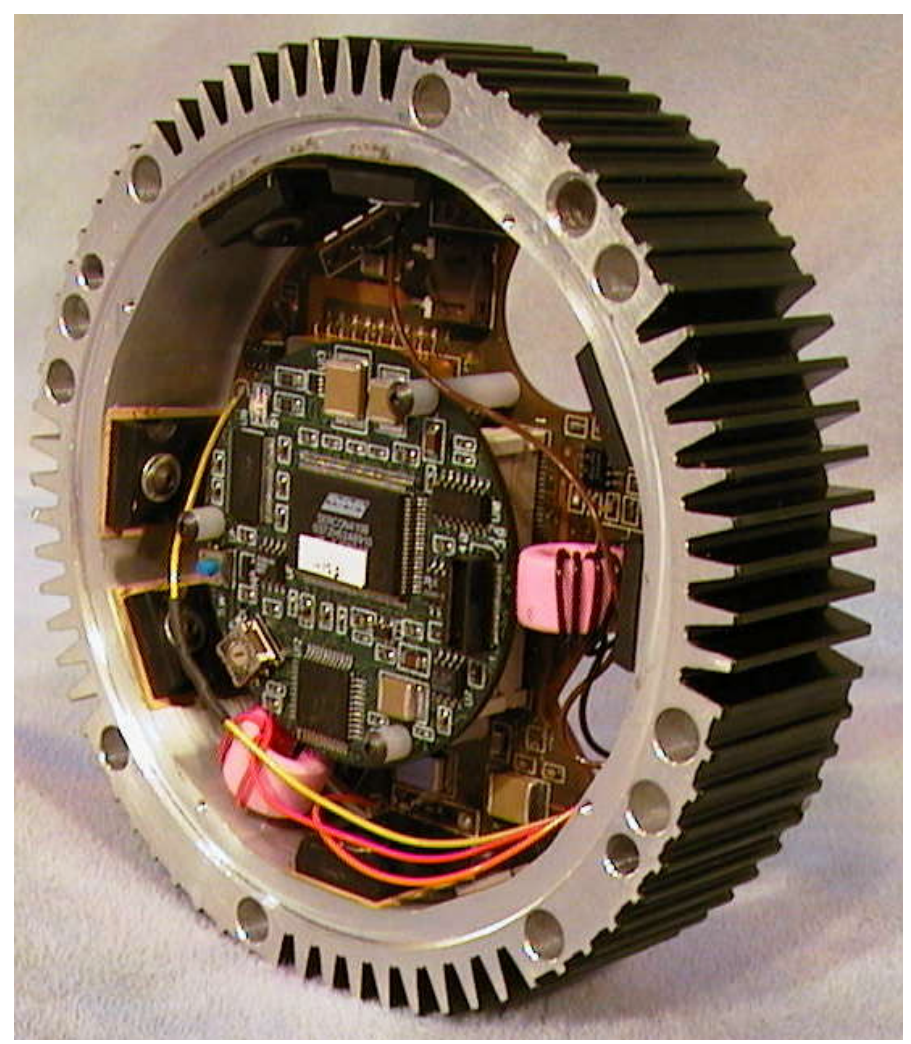

Figure 26: Completed PSI32 mounted in housing.

elements of the PSI20 design and the Power Supply and System Controller Interface (PSSCI) design. Consequently, there were very few design errors on the board. The first design error was the reversal of the positive and negative inputs into an operational amplifier used in the Main Bus Voltage Detect circuit. This was fixed by adding jumper wire to the board. Another testing issue arose in the substitution of a supposedly equivalent operational amplifier since the originally specified component was unavailable. The equivalent amplifier did not have tight enough specifications and consequently had to be changed out in favor of the original component. The acquisition of this component delayed the completion of the PSI32 board by about 2 months. Another issue arose regarding the encoder and Halls signals. The DISC ${ }^{\text {TM }}$ was receiving erroneous readings when the motor had a step in current. After some investigation, the solution resulted in a decrease in the pull-up resistance from $10 \mathrm{~K} \Omega$ to $1.2 \mathrm{~K} \Omega$. One final issue was in the height of the current sensor. The current sensor was modeled with a height of $24 \mathrm{~mm}$ but actually had a height of $25 \mathrm{~mm}$. A modification to the package successfully lowered the height of the package to less than $23 \mathrm{~mm}$.

\subsubsection{Actuator Testing}

The objective of this task was to measure the "as built" performance parameters of each actuator design. Actuator torque versus speed, accuracy and repeatability tests were performed on UTRRG's dynamometer. This dynamometer apparatus included one electronically controlled magnetic hysteresis brake. The brake was connected to a torque load cell either directly for loads less than $55 \mathrm{~N}-\mathrm{m}$ or via a gear reduction for loading greater than $50 \mathrm{~N}-\mathrm{m}$. In addition to the load-sensing device that was capable of $0.5 \%$ resolution and about $3 \%$ accuracy over full 

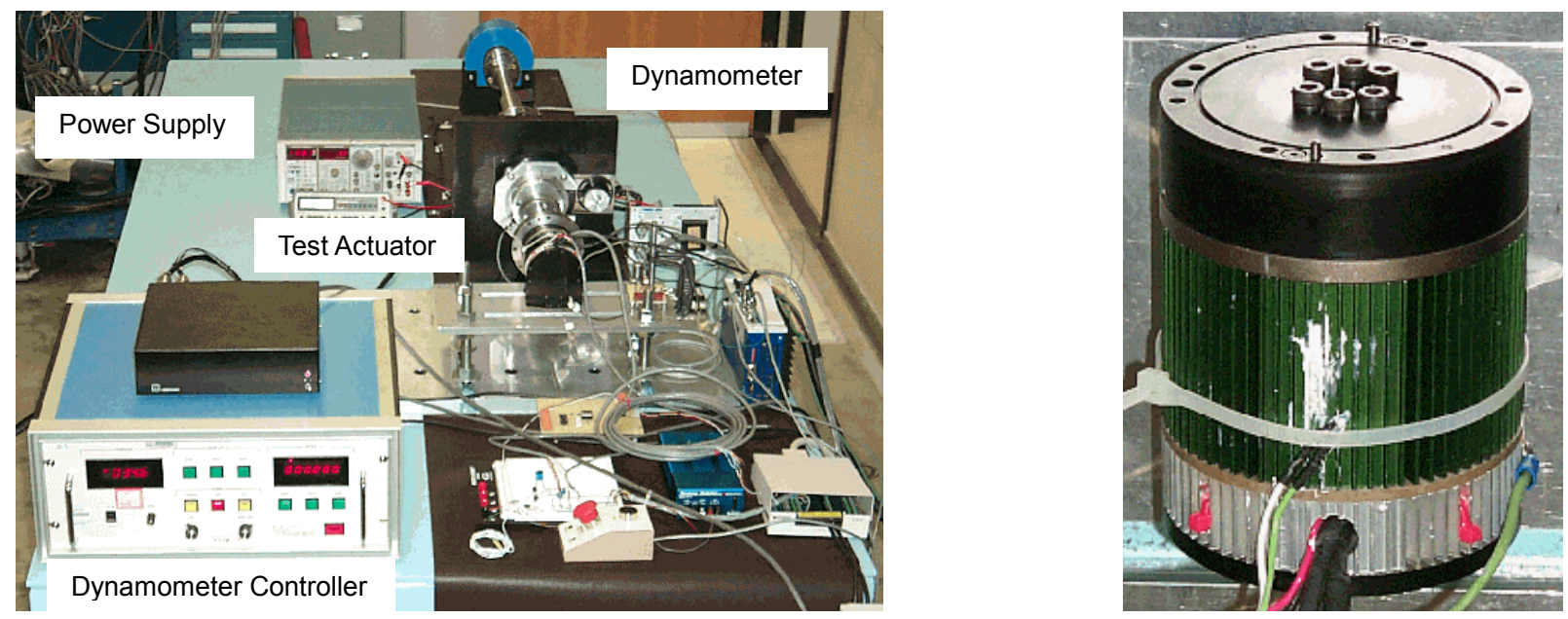

Figure 27: Laboratory test assembly and ARM20 actuator.

scale, an actuator output position sensor of ultra-high resolution was used to measure the position of the output shaft. Each actuator was mounted on a custom support frame. The actuator was controlled and monitored by a PC equipped with a DAC board running LabView ${ }^{\mathrm{TM}}$ data acquisition software.

ARM's actuator modules were provided to the UT facility fitted with temperature sensors. All actuator module tests were conducted under ambient temperatures of $25{ }^{\circ} \mathrm{C}+/-3{ }^{\circ} \mathrm{C}$. All surrounding bodies were assumed to behave as ideal blackbodies for the purposes of radiant heat transfer. Rigid thermal barriers were installed to limit conductive heat loss from the actuator through its mounts; however, considerable heat was still conducted along the shaft coupling to the dynamometer. ARM's actuator modules were tested without the integral DISC ${ }^{\text {TM }}$ amplifiers. The actuator mechanicals were controlled using an external standard industrial brushless servo amplifier manufactured by Kollmorgen provided and connected by ARM personnel.

All test measurements were recorded in SI units. The UTRRG mounted the test actuators on the dynamometer support structure and connected it to the control computer and power supply. Thermal sensors were mounted internal to the actuator and were connected to appropriate AtoD channels of the data acquisition board. The UTRRG performed tests on the actuators to determine their torque-speed curves, and their repeatability and accuracy.

The torque speed test measured the peak and continuous torque capability of the actuator as a function of speed. Figure 27 shows the test setup for this test. The continuous torque test was run by setting the actuator speed in increments of 2.5 RPM starting at 2.5 RPM and then adjusting the dynamic load until either the maximum continuous load on the gear train, the maximum current load on the motor, or the maximum system temperature was achieved. If the gear train or current was the limiting factor, the system was then run until the temperature of the system reached steady state at which point the output torque was recorded for the given speed. Figure 28 shows the measured torque-speed curve overlaid with the calculated theoretical torquespeed curve depicted in Figure 10.

To get torque vs. speed curves of the actuator, temperature measurements were used in the test procedure. Temperature rises due to changes in torque and speed were measured. The temperature effect is crucial in the performance of the actuator. Maximum limiting temperatures 


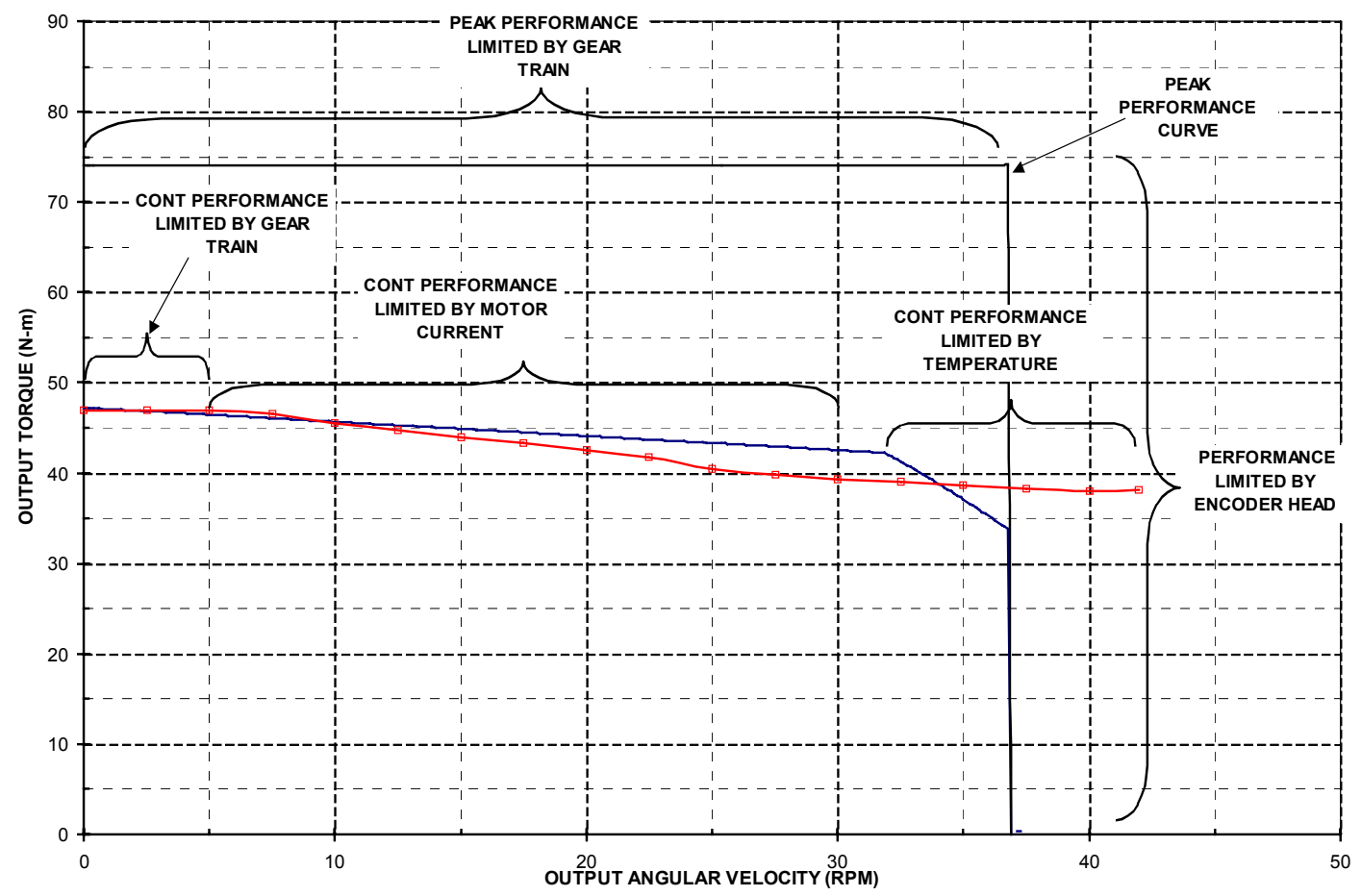

Figure 28: ARM20 rotary actuator torque vs. speed performance curve.

of components inside ARM20 are provided in Table 20. For desirable performance characteristics, these temperatures were not to be exceeded. If the actuator components are over their temperature limits, they may be overstressed, causing irreversible damage. Table 20 shows that motor coil, motor wall, and disk shell were the limiting factors on the performance of actuator and also during testing process. From the design specifications, maximum speed of the ARM20 is 36 RPM on the output due to limitations with the input encoder head count frequency. It was tested up to 42 RPM. As the result of tests, it turned out the ARM20 is capable of higher speeds providing the encoder head temperature is far below its maximum temperature. A complete report on the testing process is available in [Tesar, et al, 2001].

The measured torque vs. speed curve matched the expected curve reasonably close except the slope of the measured curve for constant current is slightly steeper than theoretical curve. This is most likely due to the additional drag load associated with the dynamometer between the motor mount and the hysteresis brake. The above test result can be interpreted as follows:

- At this initial stage, all the actuator component temperatures (such as motor coil, motor wall, motor housing, brake solenoid, disk housing, disk airspace, and atmosphere) do not

\begin{tabular}{|c|c|c|}
\hline Actuator Components & ARM 20 Max. Temp. & ARM32 Max. Temp. \\
\hline \hline Motor Coil & $150{ }^{\circ} \mathrm{C}$ & $150{ }^{\circ} \mathrm{C}$ \\
\hline Motor Wall & $125^{\circ} \mathrm{C}$ & $95{ }^{\circ} \mathrm{C}$ \\
\hline Disk Shell & $105^{\circ} \mathrm{C}$ & $95{ }^{\circ} \mathrm{C}$ \\
\hline Disk Housing & $85{ }^{\circ} \mathrm{C}$ & $85{ }^{\circ} \mathrm{C}$ \\
\hline Brake Wall & $105^{\circ} \mathrm{C}$ & $95{ }^{\circ} \mathrm{C}$ \\
\hline Brake Space & $105^{\circ} \mathrm{C}$ & $95{ }^{\circ} \mathrm{C}$ \\
\hline
\end{tabular}

Table 20: Actuator component temperature limitations used during test. 


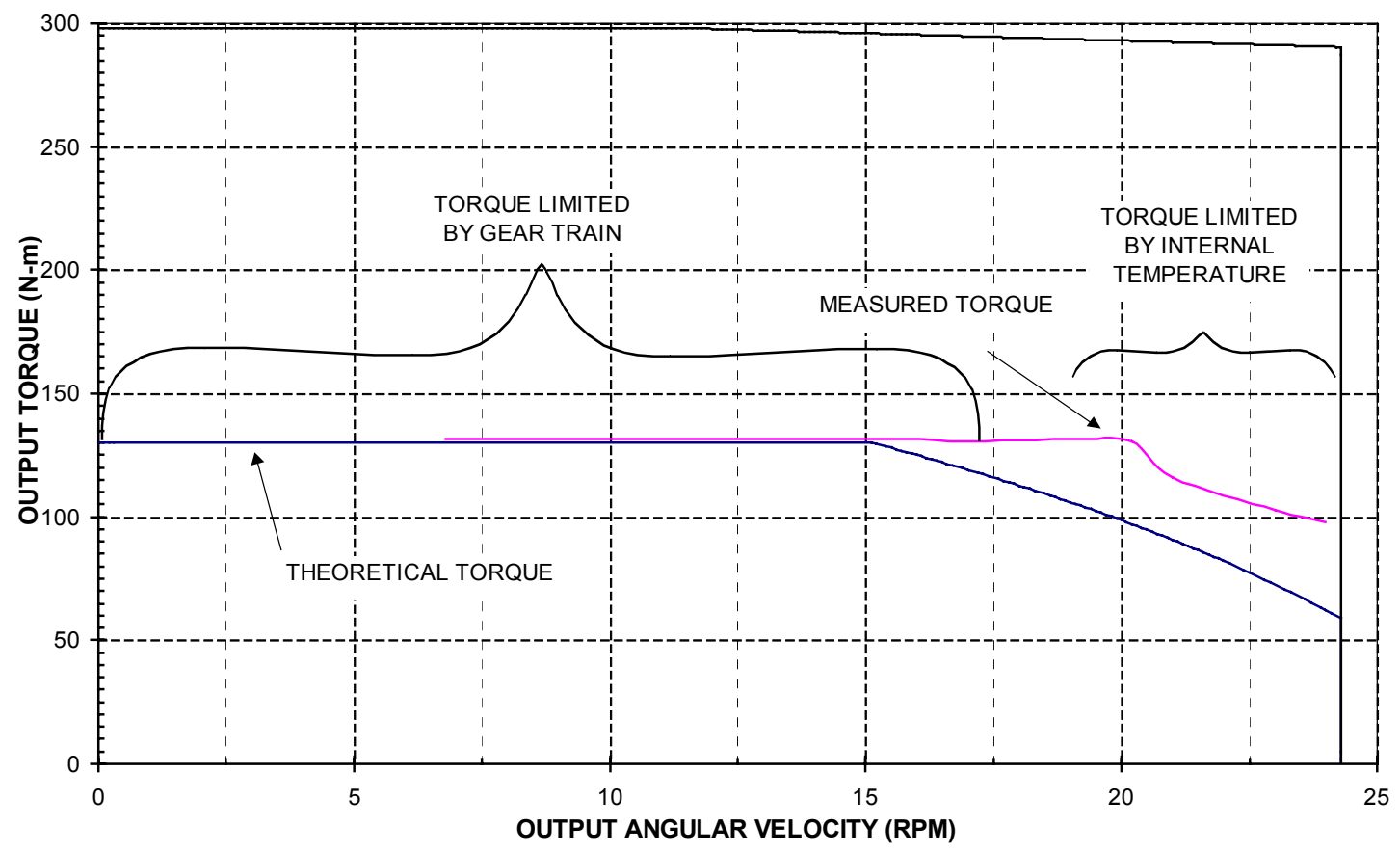

Figure 29: ARM32 measured torque-speed characteristics.

increase enough to have a significant effect on the torque vs. speed curve. The gear train is a limiting factor for actuator performance. Therefore, it determines the range of maximum output torque from the actuator at this juncture.

- At the next stage, gear train doesn't affect the actuator performance in this region. The continuous current rating of the motor effects the torque vs. speed curve and determines the range of maximum output torque from the actuator.

- At this stage, the actuator component temperatures may increase high enough to affect the performance. However, torque output is a limiting factor before the temperatures, since the torque/speed intersects the power curve. After careful study, it was determined that, in our case, the encoder used inside the actuator limited the performance. As temperature of the encoder increased, its capability of allowable count frequency decreased.

The torque speed test for the ARM32 measured the continuous torque capability of the actuator as a function of speed and the stall torque. The results found the stall torque at maximum continuous current of 2.5 Amps to be $164 \mathrm{~N} \cdot \mathrm{m}$ as compared to the theoretical value of $161.25 \mathrm{~N} \cdot \mathrm{m}$. Note that this value exceeds the continuous rating for the gear train. The test setup was similar to that for the ARM20. The continuous torque test was run by maintaining the dynamic load on the actuator at approximately $131 \mathrm{~N} \cdot \mathrm{m}$ by continuously increasing its current until the speed stopped increasing or a maximum system temperature was reached. Then, the dynamic load was adjusted until either the maximum current load on the motor, or the maximum system temperature was achieved. Figure 29 shows the measured torque-speed curve overlaid with the calculated theoretical torque-speed curve depicted in Figure 23.

To get torque vs. speed curves of the actuator, temperature measurements were used in the test procedure. Temperature rises due to changes in torque and speed were measured. The temperature effect is crucial in the performance of the actuator. Maximum limiting temperatures 


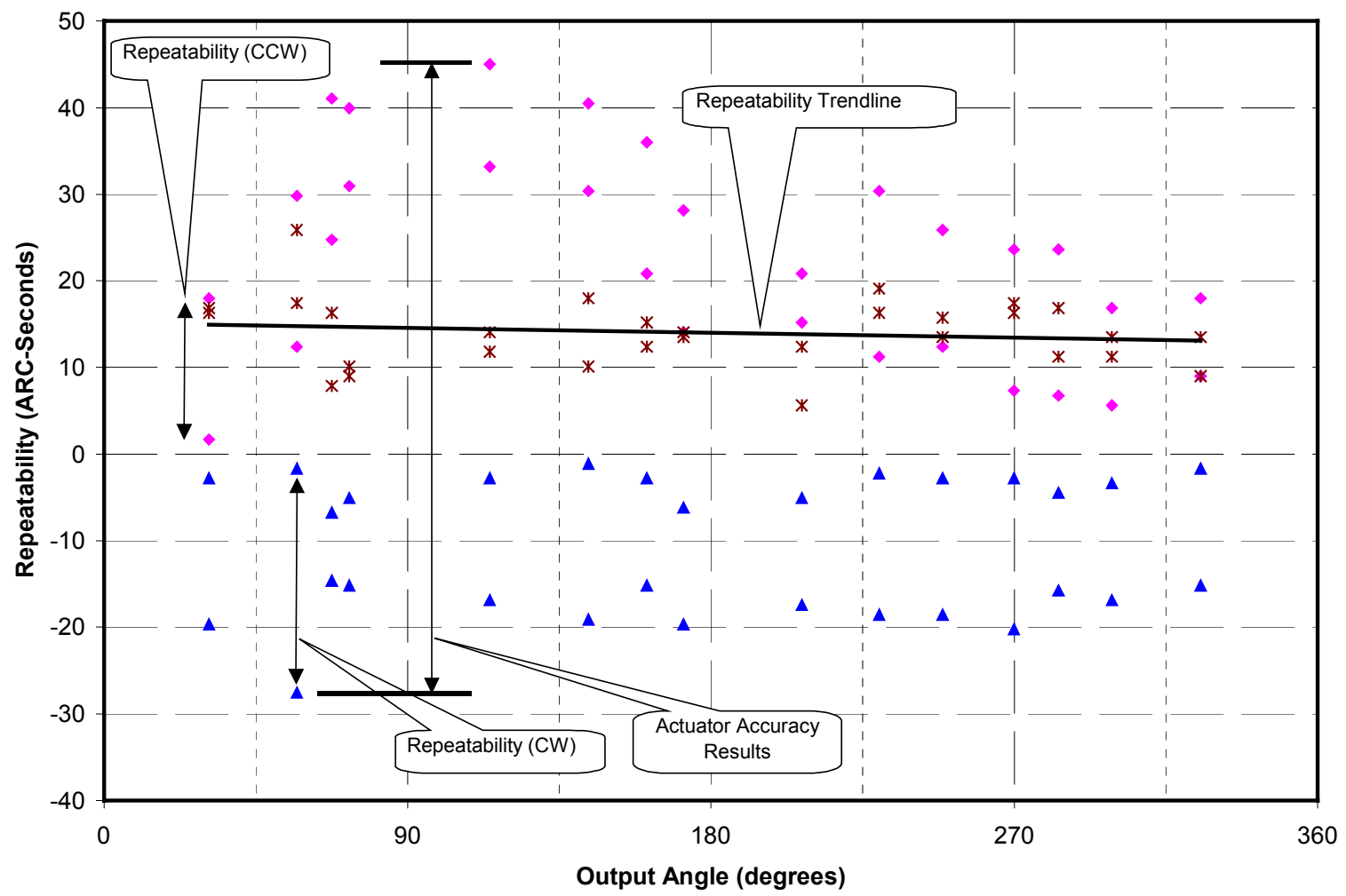

Figure 30: ARM20 repeatability and accuracy testing results.

of components inside ARM32 are provided in Table 20. For desirable performance characteristics, these temperatures were not to be exceeded. If the actuator components are over their temperature limits, they may be overstressed causing irreversible damage. Table 20 shows that motor coil, motor wall, and disk shell were the limiting factors on the performance of actuator and also during testing process. From the design specifications, maximum speed of the ARM32 is 23.4 RPM on the output due to limitations with the input encoder head count frequency. It was tested up to 24 RPM where it started to loose counts. The test results showed that the ARM32 is not capable of higher speeds.

The repeatability and accuracy test relied on acquiring multiple data points at the same point while approaching the reference point from multiple directions. Figure 30 shows the data acquired from this experiment. The repeatability is given for the worst deviation of approaching the point from the same direction. While the average was around 15 arc-seconds, the worst-case repeatability was 26 arc-seconds, which is well with the range published for the harmonic drive. The worst-case accuracy result was calculated from the maximum repeatability error less the minimum repeatability error from the commanded position. The resulting accuracy for the ARM20 was found to be 72 arc-seconds or 1.2 arc-minutes. This result is within the published range of 1.5 arc-minutes for the harmonic drive gear train which verifies the soundness of the mechanical packaging of the harmonic drive components. 

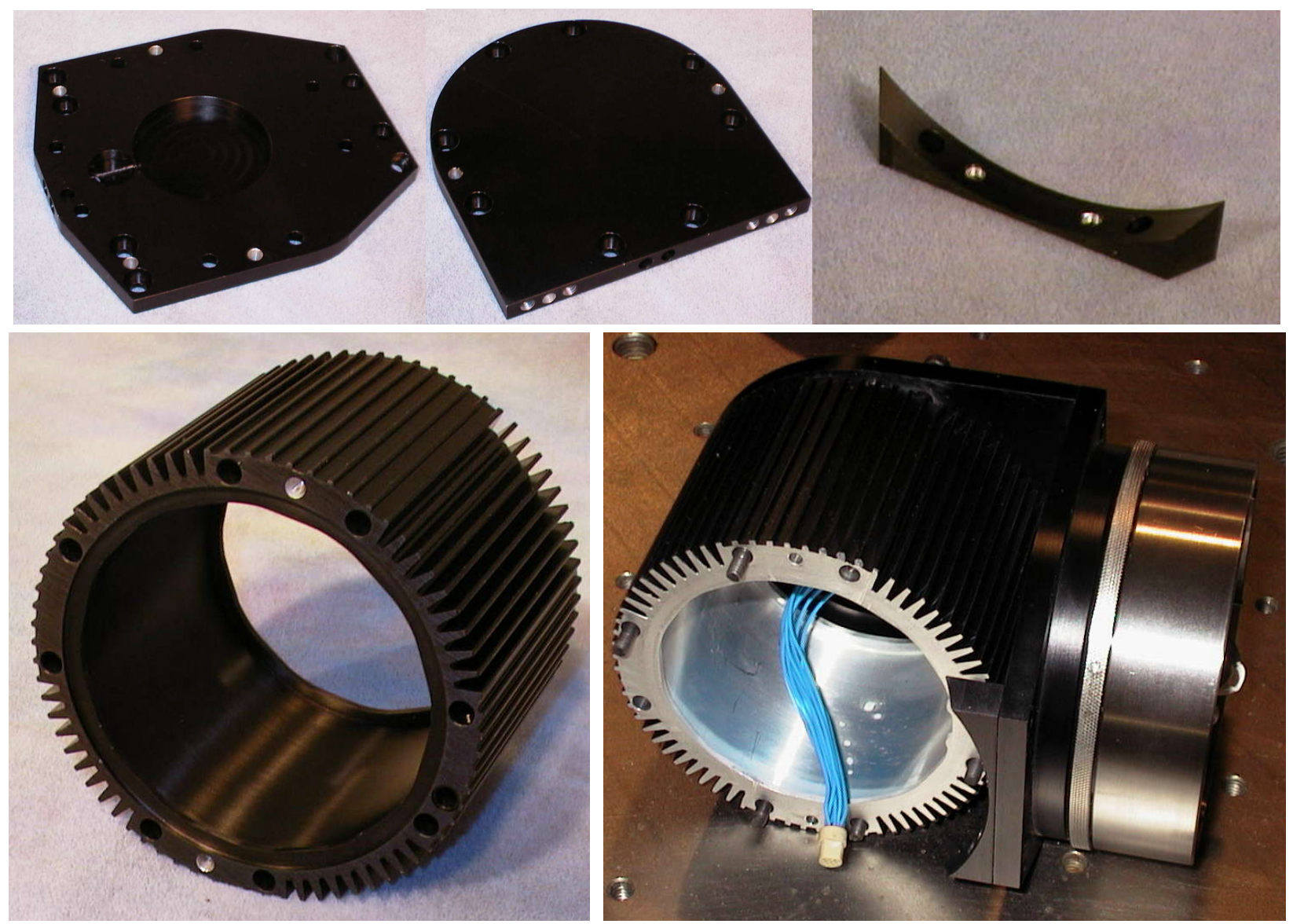

Figure 31: Yoke32 pieces: base plate (top left), end plate (top center), saddle (top right) and output extension (bottom left) and assembled Yoke20 output with a quick connect.

\subsubsection{Remaining Manipulator Components}

The remaining manipulator components to be built under this task include the yokes, the links, the quick connects, the base and end-effector plates, and the umbilical cord. Each of these systems will be discussed herein.

\subsubsection{Yoke Development}

While several yokes designs were conceptualized, a full offset was chosen for versatility. The yokes for the modular manipulator consist of two sections: output section and input section. The input section is comprised of three pieces: a base plate, an end plate, and a saddle piece. The output section consists of four pieces: the same three pieces as the input section plus an output extension used to make the output offset the same as the input. This yields a symmetric pitch joint about the interface between the input and output. The eight-bolt pattern on the output is the same as the eight-bolt pattern on the input. Figure 31 shows the yoke components for the Yoke-32 and a completed Yoke20 output section. The masses for the Yoke-20 input and output sections are $0.32 \mathrm{~kg}(11.4 \mathrm{oz})$ and $0.6 \mathrm{~kg}(21 \mathrm{oz})$, respectively, and for the Yoke-32 input and output sections are $0.87 \mathrm{~kg}(30.75 \mathrm{oz})$ and $1.55 \mathrm{~kg}(54.5 \mathrm{oz})$, respectively.

The effort to assemble the yoke on the output consists of four steps: connect the wire harness to the output of the actuator, mate the mechanical output with the dowel pins, insert and 


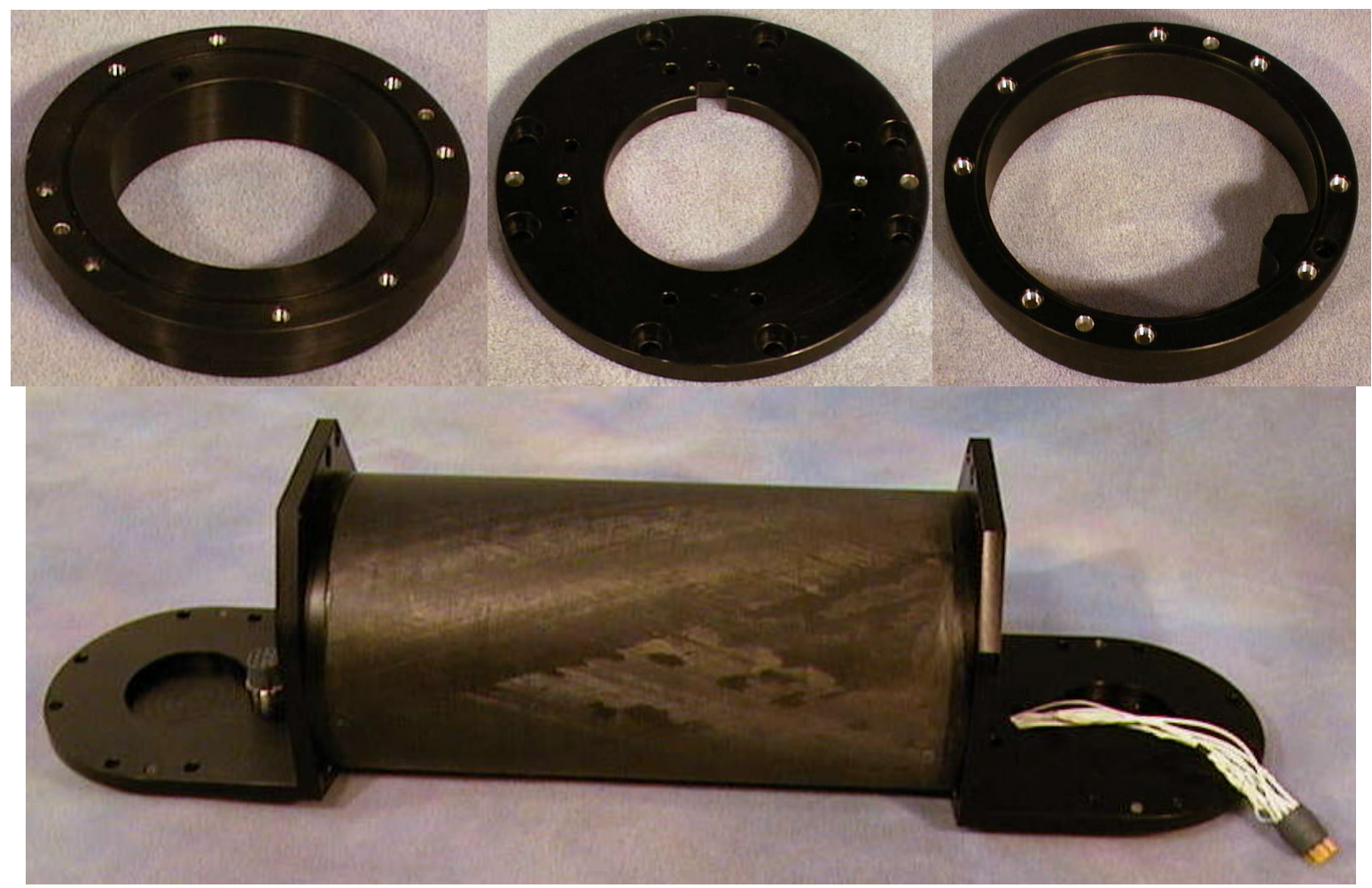

Figure 32: LINK20 flange (top left), LINK32-20 adapter plate (Top Center), LINK32 flange (Top Right) and an assembled LINK32 with Yoke32 plates (bottom).

tighten the 8 output bolts connecting the end plate to the output with the output extension, and then tighten the two saddle bolts on to the output of the actuator. The saddle piece is used to reduce the overturning moment in the end plate and is held in place by two radial bolts. These plates provide passageways the pneumatics and wire harness to/from the connecting link. The input plate has a fixed connector mounted in the end plate and the output connector has a pigtail for the connector. The ARM20 uses the same passage for the pneumatics and the wire harness. Since this proved to be too constrictive during testing, the ARM32 provides separate passageways for the pneumatics and wire harness.

\subsubsection{Link Development}

The links are comprised of carbon fiber tubes with flanges glued into both ends. The tubes have a tensile strength of $1450 \mathrm{MPa}$ (210,000 PSI) and a compressive strength of 1275 $\mathrm{MPa}(185,000 \mathrm{PSI})$ and a mass density of $1,520 \mathrm{~kg} / \mathrm{m}^{3}\left(0.055 \mathrm{lb} / \mathrm{in}^{3}\right)$. Thus, the stiffness of the carbon fiber link is about 3 to 4 times greater than that of aluminum with about half the mass density. The Link20 is designed with the same outside diameter as the ARM20 actuator and the Link32 is designed with the same outside diameter as the ARM32. These flanges have a bolt pattern identical to the actuators. For links configured for an inline roll, the flanges are designed to have the bolts tightened from the inside of the link such that they can be bolted to the actuator. Otherwise, the flanges are threaded such that they can be bolted to Yoke end plate. Figure 32 shows the threaded flanges for the Link32 and the Link 20, a Link32 to a Link20 adapter plate and a Link32 with two Yoke32s attached to either end. Each link flange is designed to pass air and electrical signals and power through it. The mass of the Link20 input flange plate is $0.15 \mathrm{~kg}$ 

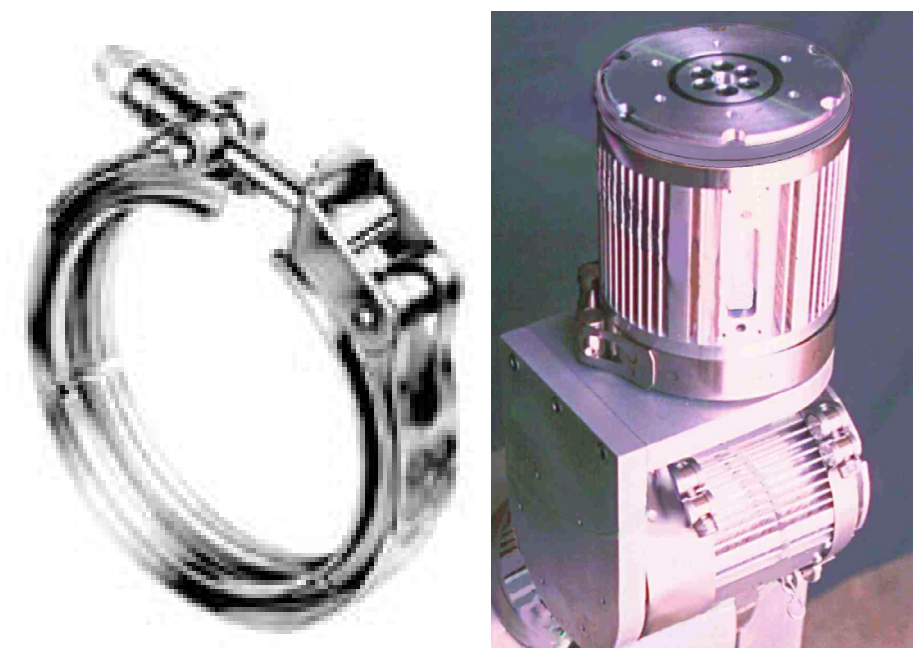

Figure 33: Typical Voss V-Band (left) and application to modular robotics (right).

( $5.4 \mathrm{oz})$ and the output flange's mass is $0.19 \mathrm{~kg}(6.8 \mathrm{oz})$. Each Link20 flange adds $10 \mathrm{~mm}$ to the length of the link when it is glued into the carbon fiber tube. The Link32 input and output flanges are identical. Their mass is $0.26 \mathrm{~kg}(9.2 \mathrm{oz})$ including the bolts for the interface. Each Link32 flange adds $9 \mathrm{~mm}$ to the length of the link when it is glued into the Link32 carbon fiber tube. The Link20 carbon fiber tube has a mass per unit length of $1.8 \mathrm{~kg} / \mathrm{m}(0.1012 \mathrm{lb} / \mathrm{in})$ and the Link32 has a mass per unit length of $1.75 \mathrm{~kg} / \mathrm{m}(0.101 \mathrm{lb} / \mathrm{in})$. The mass of the Link32-Link20 adapter plate is $0.19 \mathrm{~kg}(6.7 \mathrm{oz})$.

\subsubsection{Quick Connect Development}

The quick connect is the point of rapid assembly/disassembly for a modular manipulator. ARM originally envisioned the quick connects for the modular manipulator to consist of V-band clams similar to those shown in Figure 33. While this method of assembly is fairly economical, it is very difficult for a single person to assemble. Additionally, this approach presents pinch points that will be unacceptable for use in a glovebox environment. Consequently, ARM developed the design shown in Figure 34 that is simple for a single person to mate while minimizing the overall pinch points. The connection is designed such that the guide pins mate first followed by the electrical connector. Then the locking collar can be oriented to slip between the mating face's teeth at which point the two interfaces are pushed together. The locking collar is then rotated to hold the connection in place. The joint is cinched up by rotating the smaller ring until approximately $16 \mathrm{~N} \cdot \mathrm{m}(12 \mathrm{ft} \cdot \mathrm{lbs})$ of torque are applied to the collar. A spanner wrench can be used to apply the torque. When the collar is torqued to the specified value, the connection has approx. 20,000 $\mathrm{N}$ between the interfaces. A force of more than 12,500 $\mathrm{N}$ is required to avoid any interface separation resulting in maximum stiffness for the manipulator. The mass of the QC-20 is $0.2 \mathrm{~kg}(7 \mathrm{oz})$ for the passive flange and $0.61 \mathrm{~kg}(21.6 \mathrm{oz})$ for the active flange. The QC 20 adds an additional $42.8 \mathrm{~mm}$ (1.68 in) to the length if it is glued into the joint and an additional $46.2 \mathrm{~mm}$ (1.8 in) if it is bolted to the Link20 flange.

The only issue that arose with the quick connect design is its manufacturing cost of more $\$ 4000$ per joint. This is about 4 times the cost of a similar V-band joint. ARM also noted that while modularity has many good qualities, most systems are not reconfigured all that often. Additionally, each quick connect, whether it is a V-band or ARM's custom design, adds 


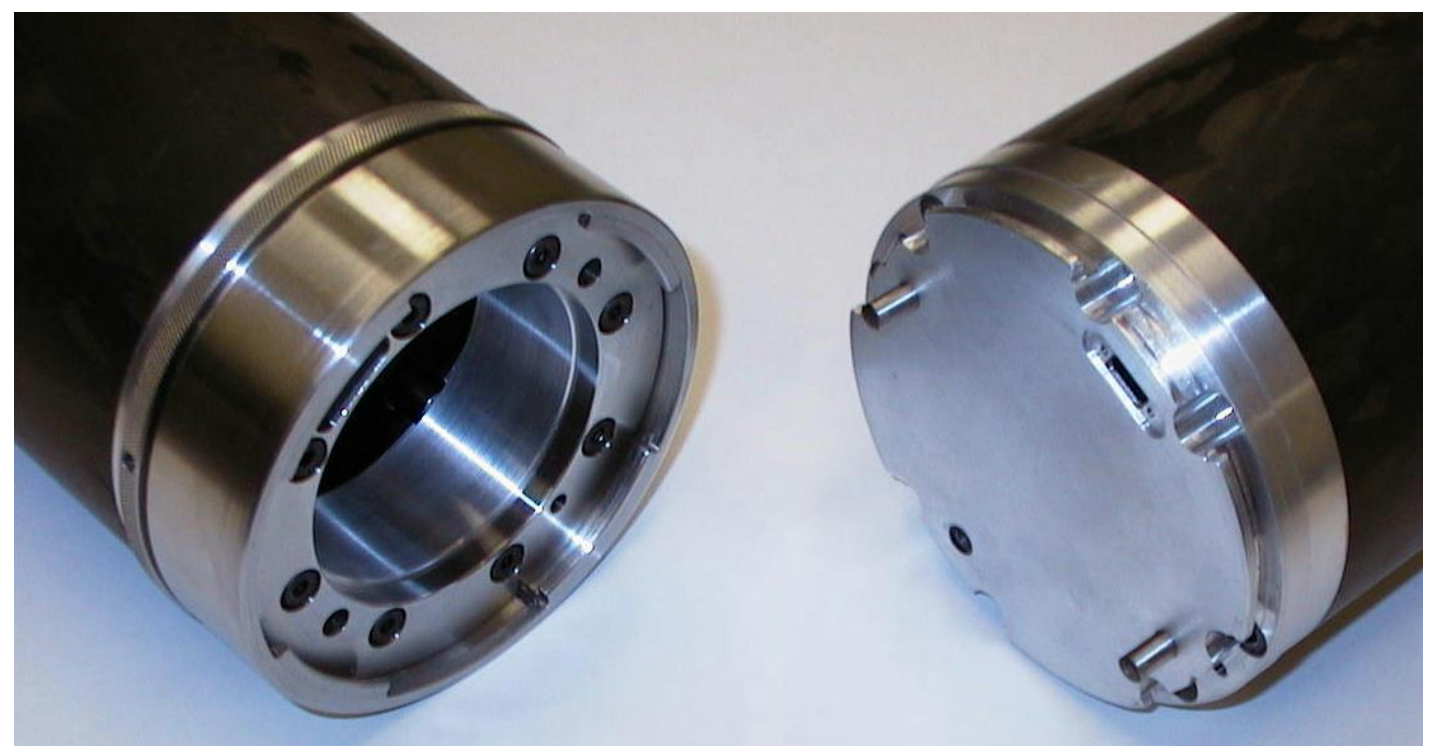

Figure 34: ARM's custom quick connect assemble.

considerable mass to the manipulator which in turns reduces its overall payload. For these reasons, ARM chose to use a bolted interface for the pitch joints while quick connects were utilized in the links. The quick connects take about 20 seconds to assemble/disassemble and the bolted interfaces take about one and a half minutes to assemble/disassemble. Thus, the current mixture of quick connects and bolted joints provide the flexibility required for a modular system with minimal additional cost. Furthermore, quick connects are necessary to facilitate assembly of the manipulator through lead-lined gloves inside the glovebox.

\subsubsection{Base And End-Effector Plate Development}

Two base plates were developed under this project. The first base plate was designed to mount to the linear track for the manipulator configuration similar to that shown in Figure 3. The second base plate was designed to mount directly to the demonstration table. These plates were primarily configured to have ARM32 actuators and associated hardware mounted to them but can be adapted to the ARM20 hardware with the use of a Link32 to Link20 adapter plate.

The gripper utilized in testing the manipulator was a TRI gripper, model number EP100/30 loaned to ARM from UT. This is a simple open/close gripper that operates from a $24 \mathrm{~V}$ supply. The voltage to the gripper was controlled from the power supply. The end plate was adapted to attach to a Yoke20 base plate. The data communications is looped back in the end-effector plate.

\subsubsection{Umbilical Cord Development}

The umbilical cord for the manipulator is comprised of a 7 wire cable, a 2 pair, shielded twisted pair cable, and a pneumatic line. The 7 wire stranded cable is an Olflex cable for the following signals: Earth Ground, Main Bus Power (2 wires), Control Power (+24 V) (2 wires), and end-effector power ( 2 wires). The twisted pair cable is for the SERCOS communications system. The length of the cord is 20 feet. 

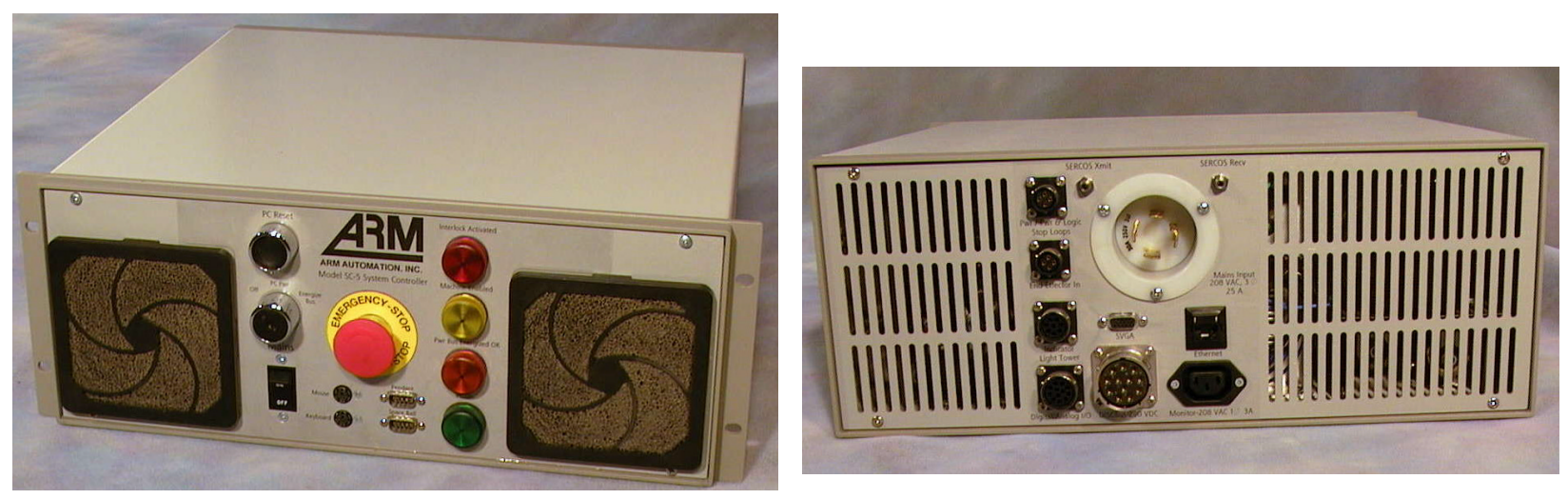

Figure 35: Front and rear panels of power supply.

\subsubsection{Integrated Power Supply And System Controller}

The power supply contains the utility interface, the main power conditioning circuitry, the control (24 V) supply, the system controller power supply, a PSSI board, relay control logic, and the system controller. The supply can be configured to interface to either single phase 120 or three-phase $208 \mathrm{~V}_{\mathrm{AC}}$. Figure 35 depicts the exterior of the supply and Figure 36 presents the interior wiring. Each of these will be expounded on in the paragraphs below.

The front panel of the power supply contains the local interface to the system controller as well as the control circuitry for bringing up the power supply. The four switches on the front panel perform the following functions: Power On/Off switch/breaker, Off/PC On/Energize High Power Bus key selector switch, PC Reset, and Emergency Stop. The switch/breaker initiates the power up of the system. The breaker is rated at 15A. This switch turns on the control power supply. The key selector switch is a 3-position switch with the following logic from left to right: Normally Open (NO) (Off), Normally Closed (NC) (PC On), and Momentary Closed (MC) with spring return to center (Energize Main Bus). When switched from the NO to the NC position, the switch turns on power to the system controller (PC) and the system controller begins its boot sequence. At the appropriate time after all the system's amplifiers have been initialized, the key switch is turned to the MC position to energize the main bus. Note that the main bus cannot be energized until the $24 \mathrm{~V}$ bus has been energized, and the safety and emergency stop loops have been closed. The front panel also has PS2 mouse and keyboard connections to the system controller. Two RS232 DB9 connections are brought out to interface to a teach-pendant and a space ball. The four lights on the front panel represent from top to bottom the state of the emergency stop loop, the machine enable, power bus energized, and PC power. The emergency stop loop light is illuminated anytime the loop is not closed. The machine enable light is activated when the system controller enables the DISC ${ }^{\mathrm{TM}} \mathrm{S}$. The Power Bus lamp is lit when there is voltage on the main power bus. The PC On light is activated when the key switch is turned to PC On. There are two fans on the front panel, each capable of moving up to $120 \mathrm{CFM}$ of air.

The rear panel has 11 connectors. The large white connector is for the connection to the utility supply. The power supply can be configured to take in either $120 \mathrm{~V}_{\mathrm{AC}}$, single phase or $208 \mathrm{~V}_{\mathrm{AC}}$, three-phase power. The four connectors on the left column are from top to bottom for the external emergency and safety stop loops, external end effector power in, light tower interface, and external signal interface. The pin configuration for each of these four connectors 


\begin{tabular}{|c|c|c|c|}
\hline \multicolumn{2}{|c|}{ Emergency and Safety Stop Loops } & \multicolumn{2}{c|}{ Light Tower Interface } \\
\hline Pin \# & Description & Pin \# & Description \\
\hline A & E-Stop Loop Out & A & For Future Functionality \\
\hline B & E-Stop Loop In & B & For Future Functionality \\
\hline C & Safety Stop Loop Out & C & For Future Functionality \\
\hline D & Safety Stop Loop In & D & For Future Functionality \\
\hline \multicolumn{2}{|c|}{} & E & For Future Functionality \\
\hline \multicolumn{2}{|c|}{ External End Effector Power (1) } & F & For Future Functionality \\
\hline A & Positive End Effector Power & G & For Future Functionality \\
\hline B & End Effector Power Return & H & For Future Functionality \\
\hline C & Not Used & & External Signal Interface \\
\hline D & Not Used & A & For Future Functionality \\
\hline (1) & Pin Configuration when Used without & B & For Future Functionality \\
\cline { 2 - 4 } & External Isolation Transformer & C & For Future Functionality \\
\cline { 2 - 4 } & & D & For Future Functionality \\
\cline { 2 - 4 } & & E & For Future Functionality \\
\hline \multicolumn{2}{|c|}{ External Isolation Power Transformer (2) } & F & For Future Functionality \\
\hline A & Isolated Main Power In 1 & G & For Future Functionality \\
\hline B & Isolated Main Power In 2 & H & For Future Functionality \\
\hline C & Main Power Out 1 & I & For Future Functionality \\
\hline D & Main Power Out 2 & J & For Future Functionality \\
\hline (2) & Pin Configuration when Used with External & K & For Future Functionality \\
\cline { 2 - 4 } & Isolation Transformer & J & For Future Functionality \\
\hline
\end{tabular}

Table 21: Pin assignment for power supply's rear panel connectors.

is located in Table 21. The emergency and safety stop loop connector allow external loops to be closed remotely from the system controller. If this functionality is not desired, then a loop back plug must be used for the system to function properly. The external end effector power connector's originally intended use was for supplying external power to the end effector; however, ARM wired this connector to interface to an external isolation transformer to step up $120 \mathrm{~V}_{\mathrm{AC}}$ to $208 \mathrm{~V}_{\mathrm{AC}}$. This allows the power supply to interface to a normal wall outlet at reduced power that was necessary for remote demonstrations since $208 \mathrm{~V}_{\mathrm{AC}}$ three-phase was not often readily available. The external light tower interface outputs the same four signals that are represented on the front panel. The external signal interface allows the system controller to interface to local digital and analog I/O signals. The two external FSMA fiber optic connectors are for closing the SERCOS loop with an external SERCOS amplifier. The SVGA connector is for interfacing the System Controller with a local monitor. Power for the monitor is available on the bottom just below the Ethernet connector. The Ethernet connector is for placing the system controller on a local network. This is necessary to remotely operate the system controller. The 12-pin interface for the umbilical cord is located on the bottom center.

Figure 36 shows the internal wiring of the system controller and power supply. The power supply contains the utility interface, the system controller, the control power supply $(24 \mathrm{~V})$, the main power supply, the robot relay ladder logic, and a power supply system interface board. Each of these aspects will be discussed below. 


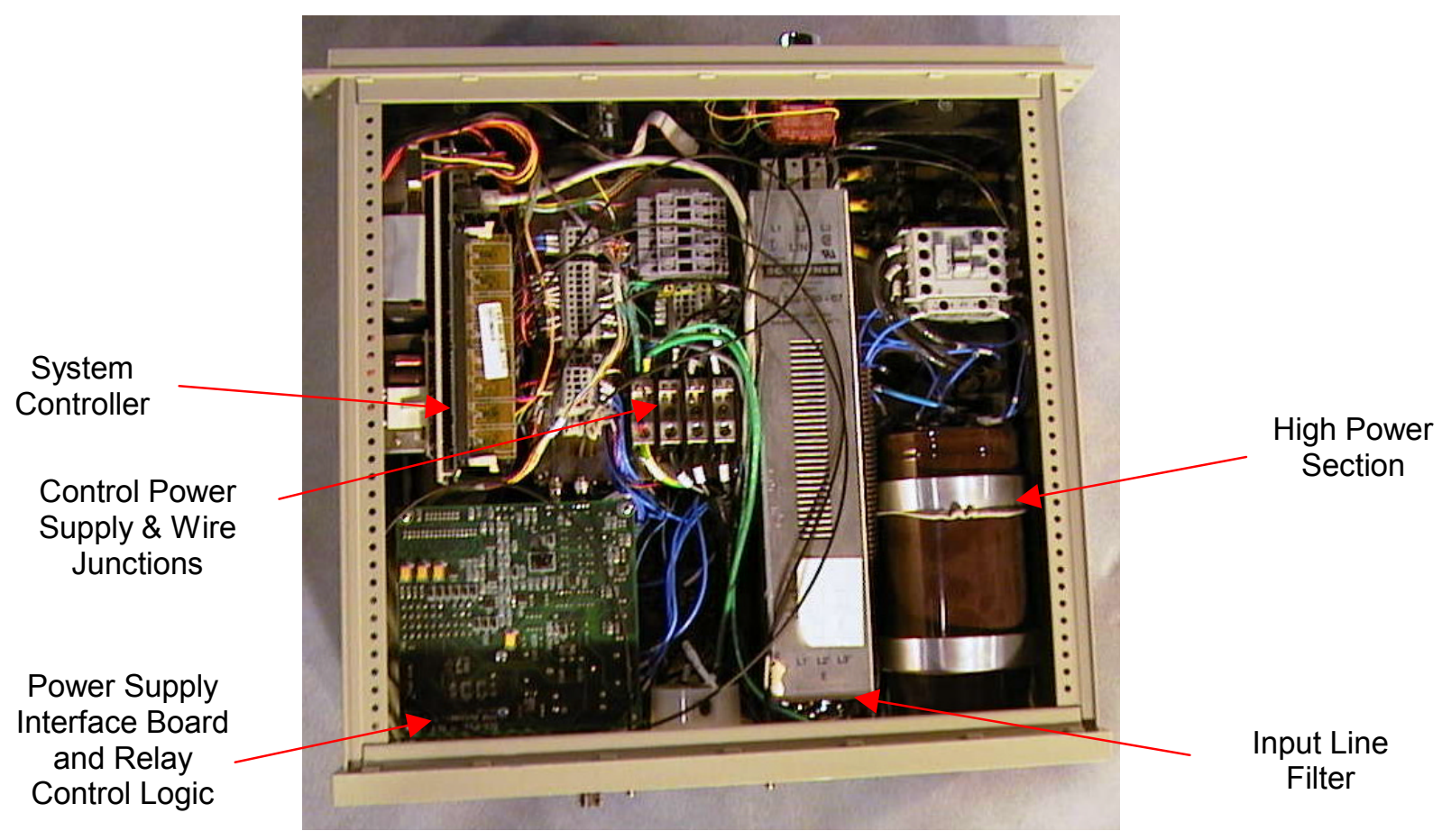

Figure 36: Power supply's internal configuration.

The utility interface was originally designed for three-phase $208 \mathrm{~V}_{\mathrm{AC}}$. However, due to constraints for demonstrating the system at DOE conferences, the supply was rewired to accommodate $120 \mathrm{~V}_{\mathrm{AC}}$ single-phase input. To allow for the proper voltages to the motors, an external step-up isolation transformer was used to step the $120 \mathrm{~V}_{\mathrm{AC}}$ up to $208 \mathrm{~V}_{\mathrm{AC}}$ for the main power supply voltage. A CE rated filter filters the input line voltage to prevent internal noise being put back on the mains. The line power is limited to $15 \mathrm{~A}$ by a combination breaker switch located on the front panel. The line input is then fused going into the fans, the control power supply, the external monitor plug, the system controller power supply, and the main power supply.

The system controller is a single board computer consisting of an Ethernet controller, an IDE controller, a PC-104 expansion bus, several digital I/O and analog input lines, and a Pentium Class processor with $64 \mathrm{M}$ of memory. There is a 4 Gigabyte hard drive connected to the IDE controller. An MEI SERCOS motion card is resident on the PC-104 bus. This card is used to control the trajectories on the individual axes. The system controller's operating system is NT 4.0. The open-architecture system controller software used for this task is Cimetrix.

The control power supply is a $24 \mathrm{~V}, 400 \mathrm{~W}$ power supply. It supplies power for the power supply and system interface board, relay ladder logic, light tower, robot control supply, and end effector. The system controller monitors the current supplied to the end effector and control bus.

The main power supply is designed to function from 60 to 320 Volts, DC. It is designed to deliver up to $16 \mathrm{~A}$ of current. It can store over 10 Joules of regenerative energy for a voltage rise from 320 Volts to 355 Volts before it dumps the dynamic braking energy from the motors into power resistors thermally isolated underneath the storage capacitor in the high power section. The PSSI board controls the dumping of this energy. The PSSI board also controls the soft starting of the main power bus for initially charging the large capacitor up to the main bus voltage. 
The PSSI board provides the glue logic for the power supply, the relay logic, the system controller, and the communications. The board has the following functionality: System controller interface, general purpose external digital I/O and analog inputs, front and real panel indicator lamp control, SERCOS fiber optics signal to RS-485 conversion, internal power supply signal conditioning, relay ladder logic drivers, end effector control, and end effector current sensing, ground fault sensing, main power bus soft starting, over-voltage sensing and dynamic braking, and board power conditioning. All system controller digital interface signals are optoisolated from the conditioned signals on the PSSI board. There are 3 general-purpose inputs and two general-purpose outputs from the system controller that are connected to the output. There are also two analog inputs that can also be used for general purposes. These signals are connected to the back panel of the power supply. The analog signals are not opto-isolated. The SERCOS signal is converted from fiber optics to RS-485 for transmission to the ARM actuator modules. This conversion was necessary to reduce the complexity of the wire harness traversing through the manipulator. The return signal is then converted back to a fiber optic signal and transmitted to the rear of the power supply where it can communicate with external SERCOS devices. The external return is then attached to the MEI motion card.

The relay ladder logic is designed with a specific order in which the robot is to be energized per [RIA, 1999]. After the breaker is switched on, the first relay is engaged by turning the key switch to the center position. This turns on the power to the system controller, which then initiates its boot sequence. At this point the green PC On light on the front panel is lit. The next rung on the ladder logic is the emergency stop loop (E-Stop). This loop consists of a serial chain of contacts with inputs from the external emergency stop, the system controller on the PSSI board, the PC power relay, the emergency stop button on the power supply, and the supply's case interlock switch. If any one of these contacts is opened, then the E-Stop is open causing all rungs after the E-Stop to also be open. The red light on the system controller front panel is lit when this loop is opened. As soon as the E-Stop loop is closed, the system controller can control the end effector on/off and power select relays and the control power supply relay to the manipulator. The manipulator's safety stop loop can be closed as soon as the control power is available to the manipulator. The safety stop loop is used to remove the motor power from the manipulator. The system controller, an over-current on the main or control power bus, or an external safety stop can break it. The main bus is then powered up by turning the key switch clockwise to the momentary position. As soon as the bus is power up to the full voltage, the orange light on the front panel is lit. The machine enable light can then be turned on and off by the system controller software.

The system controller coordinates the motion of the joints of the modular robot. The control architecture chosen for this project was an open-architecture system developed by Cimetrix. This controller is client-server format with the server running in the power supply and the client running either on the power supply or externally and communicating to the server using a TCP/IP protocol (see Figure 37). The client generates the manipulator's joint set points and passes them to the server. The server then generates the desired motion profile and passes the commands on to a motion card interface. The server monitors external signals through the I/O signal interface. The CODE (Cimetrix Open Development Environment) interface is a set of library function calls that creates the interface between the user's application environment and the server. The user develops his/her application in $\mathrm{C}$, Visual $\mathrm{C}++$, Visual Basic, or Delphi using library CODE function calls supplied by Cimetrix. These function calls then pass 


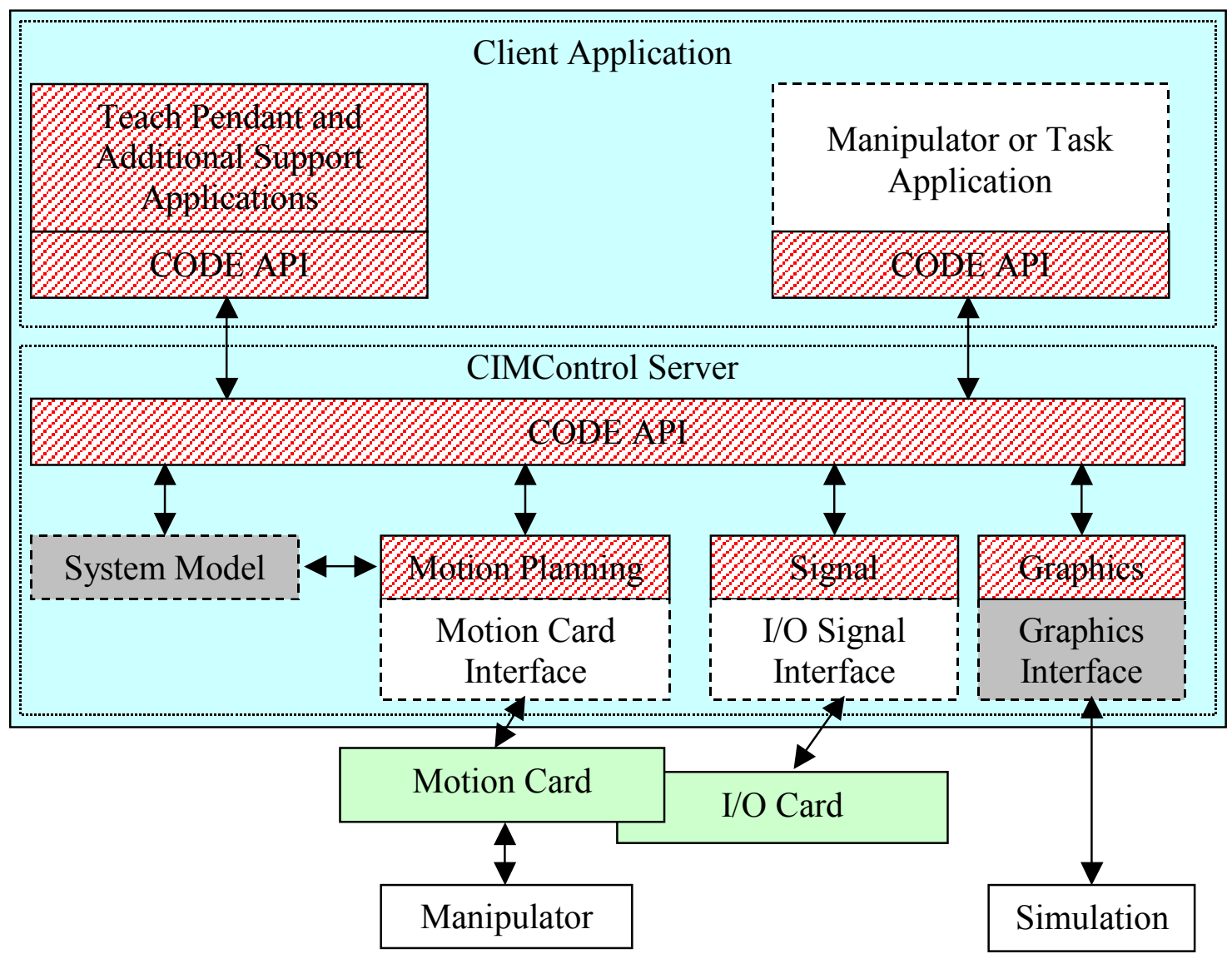

Figure 37: Block diagram of Cimetrix control hierarchy.

information to the server. The server is a multi-threaded program operating on an NT 4.0 platform that performs the motion control, error monitoring, and I/O control. The server then passes motion commands to the motion $\mathrm{I} / \mathrm{O}$ card interface.

As Cimetrix did not have an interface completed for the MEI PC-104 SERCOS card, ARM wrote this interface. This effort required the enhancements to four software modules for driving the MEI motion card supplied by Cimetrix: rob_to_mei, mei_homing, mei_config, and mei_param. The changes to the rob_to_mei module ignored MEI motion card errors until the manipulator was properly homed. This was necessary because the MEI card only reset its integral parameters on one of two conditions: reset or error. Thus, after the SERCOS drive is homed, an error needs to be thrown on one of the drives to reset the card's control parameters due to the change in position. The mei_homing module needed routines for performing drive controlled homing procedures over the SERCOS network. The mei_config module is responsible for initializing the MEI card. Thus, ARM added code to this module to initialize the SERCOS ring whenever the card gets initialized. The mei_param needed the code to turn on the front panel light whenever the robot is enabled.

\subsection{UT Software Enhancements}

The UTRRG has developed application software for tele-operation of ARM's modular robotic arms. The system uses a Magellan 6 DOF hardware device for the manual controller 
input. The application software transfers the desired end-effector location from the input device to the Cimetrix controller, which is used in the control hierarchy of the ARM manipulator. The application software is built using the OSCAR software library originally developed by UTRRG but the robot is not controlled using OSCAR. The application software is based on a specified 6 DOF robot configuration of the ARM modules operating inside of a specified glovebox workspace. However, due to the generality of the software, any configuration of modules used to build a 6 DOF robot may be controlled by simply changing the data file in the application software containing the geometric parameters for the robot. The geometric parameters are also referred to as the Denavit-Hartenberg (DH) parameters. With a bit more effort, robots with varying numbers of DOF can be controlled by changing the inverse kinematics routine specified in the application source code, although this feature does not exist in the delivered application software.

The workspace of the glovebox is modeled in a static, world-model representation. Updates to a changed glovebox environment require modifying a data file containing world model information. The collision avoidance software is therefore currently limited to a static glovebox environment.

To run the demonstration, the following executable software files must be executed in order: manual controller interface, Cimetrix Cimulation (and desired workspace), and main demonstration program. Following the initialization of the software, the Magellan 6 DOF manual controller can then be used to command the robot end-effector position within the glovebox workspace. The rate of motion is automatically slowed near obstacles. If the manual controller commands the robot such that a collision with the glovebox could occur, the collision avoidance algorithms filter out the violating commands. For example, when the portion of the command causing a collision is filtered out, the operator can move along a wall at a specified distance without danger of collision.

Functional testing of the application software through simulation was successful. Control of the manipulator was intuitive and simple, and the application software prevented all potential workspace collisions. The initial application software under delivery is a base starting point for tele-operation of reconfigurable manipulators. As the application software is used for a variety of ARM's module configurations, a library of additional DH parameter files and workspace modeling files need to be created for use with the application software.

\subsection{Manipulator Testing Results}

The manipulator testing was performed with several different configurations. The first configuration was comprised of 3 ARM20 modules and a linear track operated by a Pacific Scientific SERCOS motor/amplifier pair (See Figure 38 (a)). The use of the Pacific Scientific's amplifier demonstrated the interoperability of the amplifiers with the SERCOS specification. This configuration was used to both develop and debug the Cimetrix software and enhance and test the DISC ${ }^{\mathrm{TM}}$ software. The second configuration used both the ARM20 and ARM32 components and demonstrated the complete capability of ARM's modular approach (See Figure 38 (b)). A brief discussion of each configuration will be given below.

The first configuration used three ARM20s, two QC-20s, associated Link20s and Yoke20s, and a 1.5 meter linear track mounted on a precision tabletop. The configuration allowed for the testing and qualification of the changes made to Cimetrix, the DISC ${ }^{\mathrm{TM}}$ software, and the overall modular concept. The changes incorporated into Cimetrix primarily involved the 

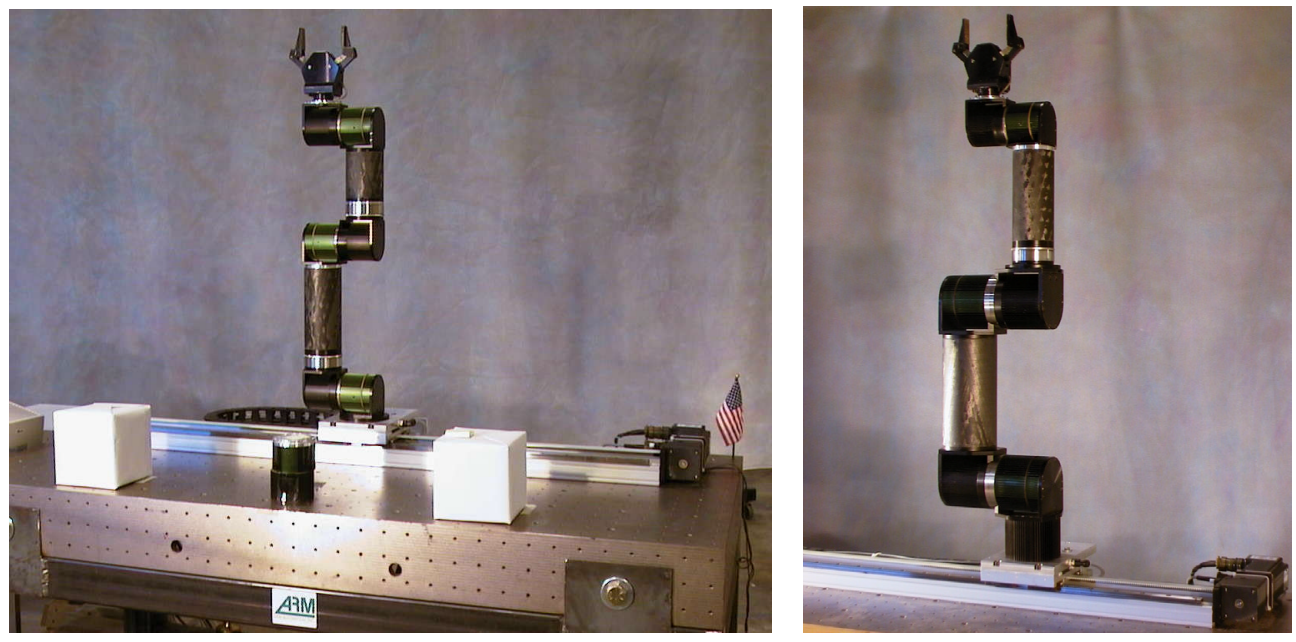

Figure 38: Manipulator configuration \# 1(a) and configuration \#2 (b).

development of the MEI interface for SERCOS as Cimetrix only supported interfaces to analog motion control cards. Additionally, the homing routine for the DISC ${ }^{\mathrm{TM}}$ was configured to behave like an analog amplifier such that the Cimetrix teach-pendant routine could be utilized.

After all software was completed, an application program was developed to test the modular concept. This test consisted of the manipulator pulling two screwdrivers from holes located on the tabletop and placing them in adjacent holes. Then the manipulator would go to a set of dial indicators and measure its position in the $\mathrm{X}$ and $\mathrm{Y}$-axes, then it would return the screwdrivers to the original position and return to the dial indicators to re-measure its point in space. The positive $\mathrm{X}$-axis is in the horizontal direction from the base of the robot towards the front of the table and the positive $\mathrm{Y}$-axis is in vertical direction coming up out of the tabletop. This test measured the manipulators repeatability, DH parameters, and ability to follow a path. The last two parameters were measured by the ability of the manipulator to complete the task of placing the screwdrivers into the holes by following a linear path along the z-axis until the screwdriver was completely in the hole. The linear path was generated from the inverse of the manipulator. Figure 39 presents the repeatability results. The test consisted of four parts: 1) initialize the two dial indicators and take data points for ten cycles; 2) disassemble and assemble manipulator and take data points for ten more cycles; 3) disassemble and assemble manipulator for a second time and take data points for ten more cycles; and 4) wait for the following day, disassemble and assemble manipulator for a second time and take data points for ten more cycles. The four batches of repeatability data given for the manipulator being dismantled and reassembled demonstrate the accuracy associated with the quick connect interfaces. For each test, the manipulator was repeatable to within \pm 3 thousands of an inch. The accuracy of the manipulator from the disassembly and reassembly operation was \pm 18 thousands of an inch. The payload capacity of this configuration was measured to be $1.5 \mathrm{~kg}$, including the mass of the gripper $(1.5 \mathrm{~kg})$, which matches its theoretical capacity with Joint 1 limited to maximum range of $\pm 135^{\circ}$. With this load, the motor reached a maximum temperature of $79.2^{\circ} \mathrm{C}$ and the DISC ${ }^{\mathrm{TM}}$ reached a steady state temperature of $85.0{ }^{\circ} \mathrm{C}$. The mass of the three DOF sections of the manipulator was $14.6 \mathrm{~kg}(\sim 32 \mathrm{lbs})$.

For the second manipulator configuration, the manipulator was tested assembling a can in can from the ARIES line under development at LANL. The repeatability required of the 
4 DOF Manipulator Repeatability Data

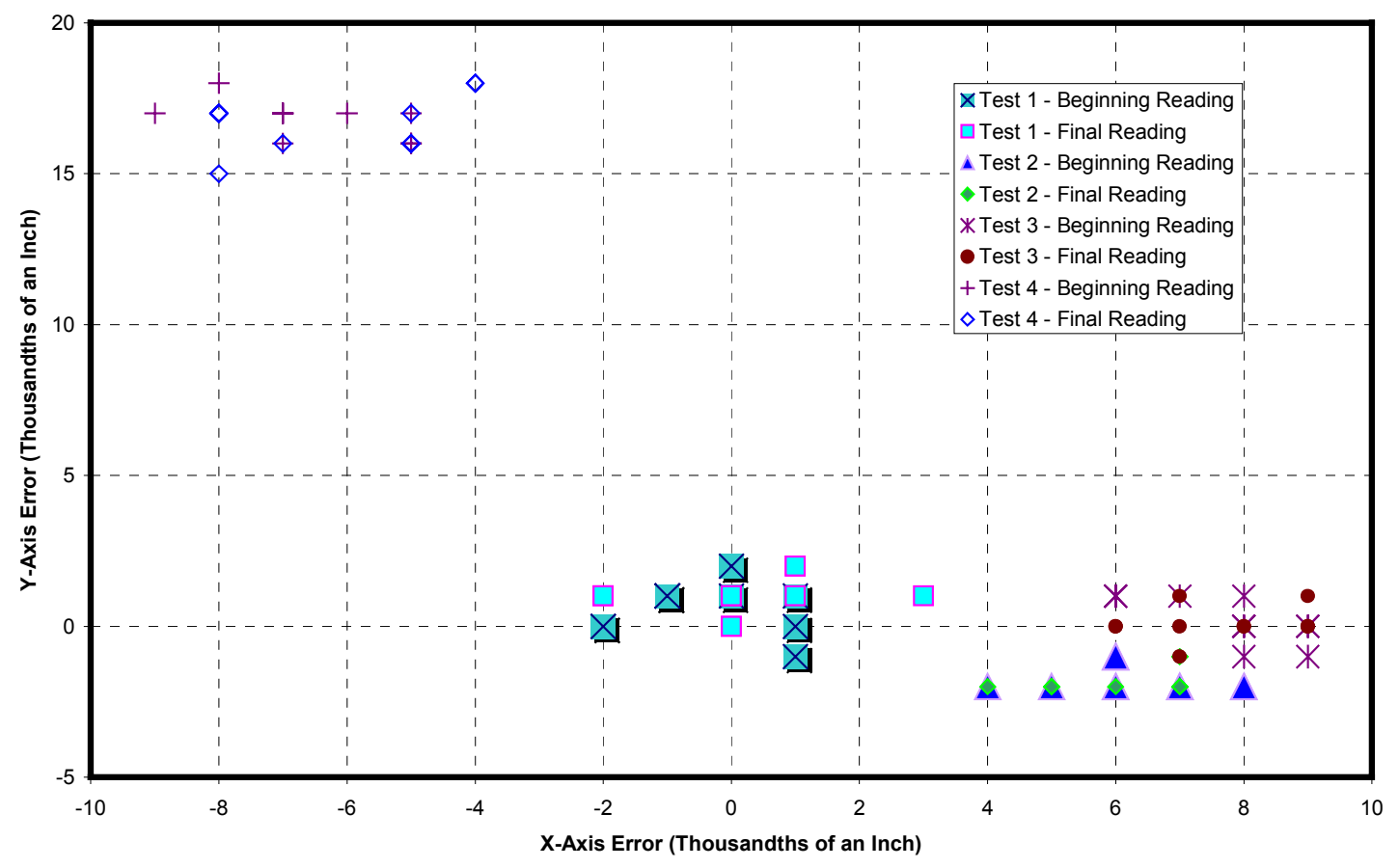

Figure 39: Repeatability data for 4 DOF manipulator.

manipulator was $\pm 0.3 \mathrm{~mm}(0.012 \mathrm{in})$. The maximum payload capacity for this configuration was the assembled container which was measured to be $5.1 \mathrm{~kg}$, excluding the mass of the gripper (1.5 $\mathrm{kg}$ ). This represented $70 \%$ of its theoretical wrist payload capacity of $9.5 \mathrm{~kg}$ with Joint 1 limited to maximum range of $\pm 135^{\circ}$. The mass of the three DOF sections of the manipulator was 25.6 $\operatorname{kg}(\sim 56 \mathrm{lbs})$.

\subsection{DOE Demonstration And System Quantification}

The system demonstration consisted of two parts: 1) demonstrating the usefulness of the quick connects for passing the modules through the glove port and then assembling the manipulator and 2) demonstrating the assembly of a convenience can inside a stainless steel primary can.

\subsubsection{Assembly Of Manipulator Using Quick Connects}

Figure 40 depicts the assembly sequence of the smaller modular manipulator shown in Figure 38 using quick connects. A joint, link and half of a quick connect are assembled external to the glovebox and then passed into the glovebox through a glove port. The pieces of the manipulator are then assembled in the glovebox by the following procedure:

1. Locate retaining ring in open position.

2. Pick up adjoining module assembly and insert in quick connect by aligning dowel pins.

3. Turn retaining ring into closed position.

4. Used spanner wrench to secure link in place by turning the preload ring. 

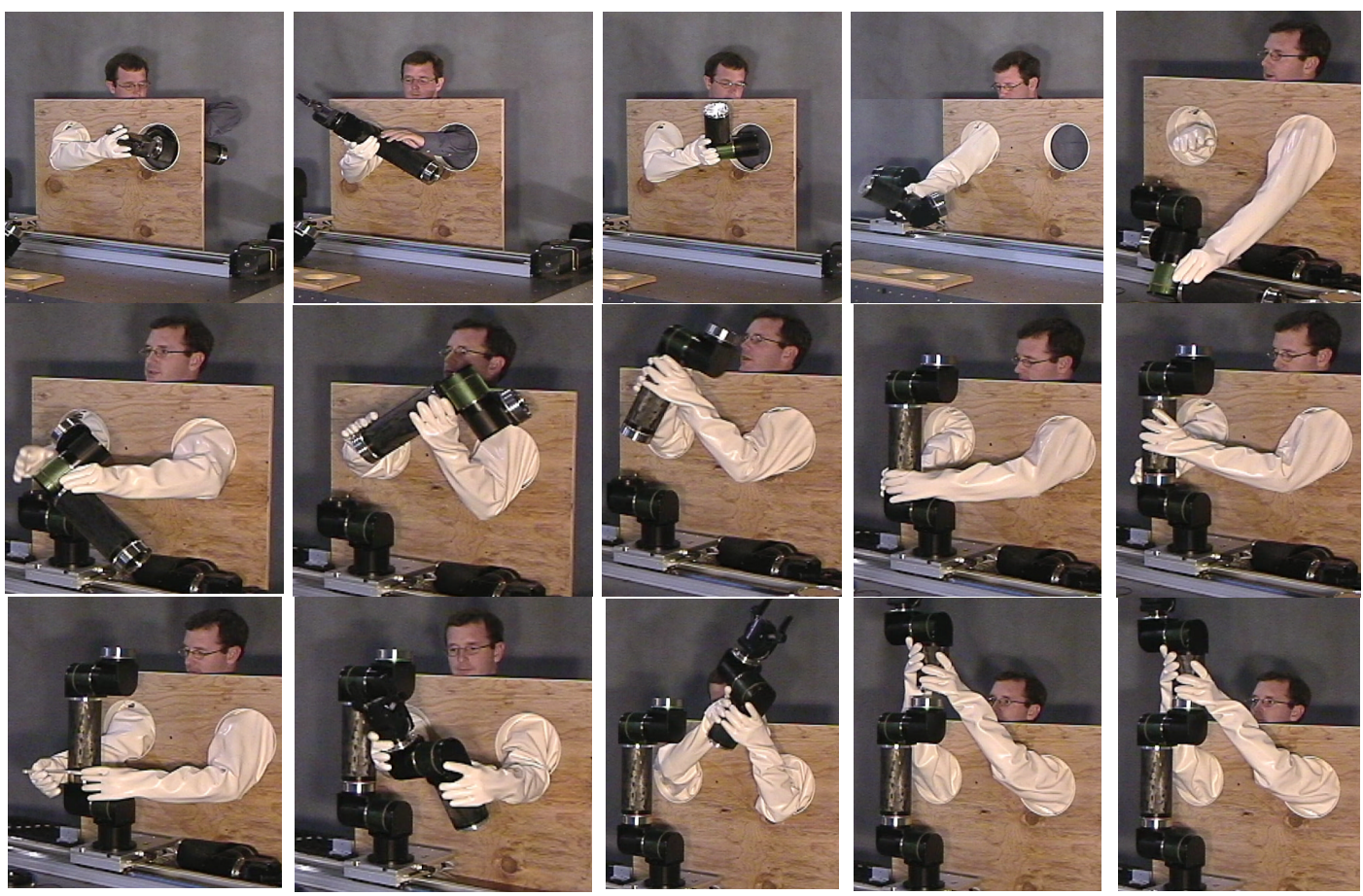

Figure 40: Assembly sequene of the smaller 4 DOF manipulator using gloves.

5. Repeat with next joint.

The simplicity of the quick connects allows a manipulator to be assembled/disassembled in a matter of minutes. Since the quick connect is a very accurate interface, a manipulator can be assembled external to the glovebox and then fully qualified before it is disassembled and passed into a confined environment. This demonstrated the first objective of this project in that a modular manipulator could be passed through a glove port, and reassembled using lead lined gloves.

Figure 41 shows the assembly sequence for the larger manipulator shown in Figure 38 (b) from passing the components through a glove port to the assembly of the manipulator. This demonstrated that the ARM32 modules and links can also be passed through the glove port and the assembled internal to the glovebox.

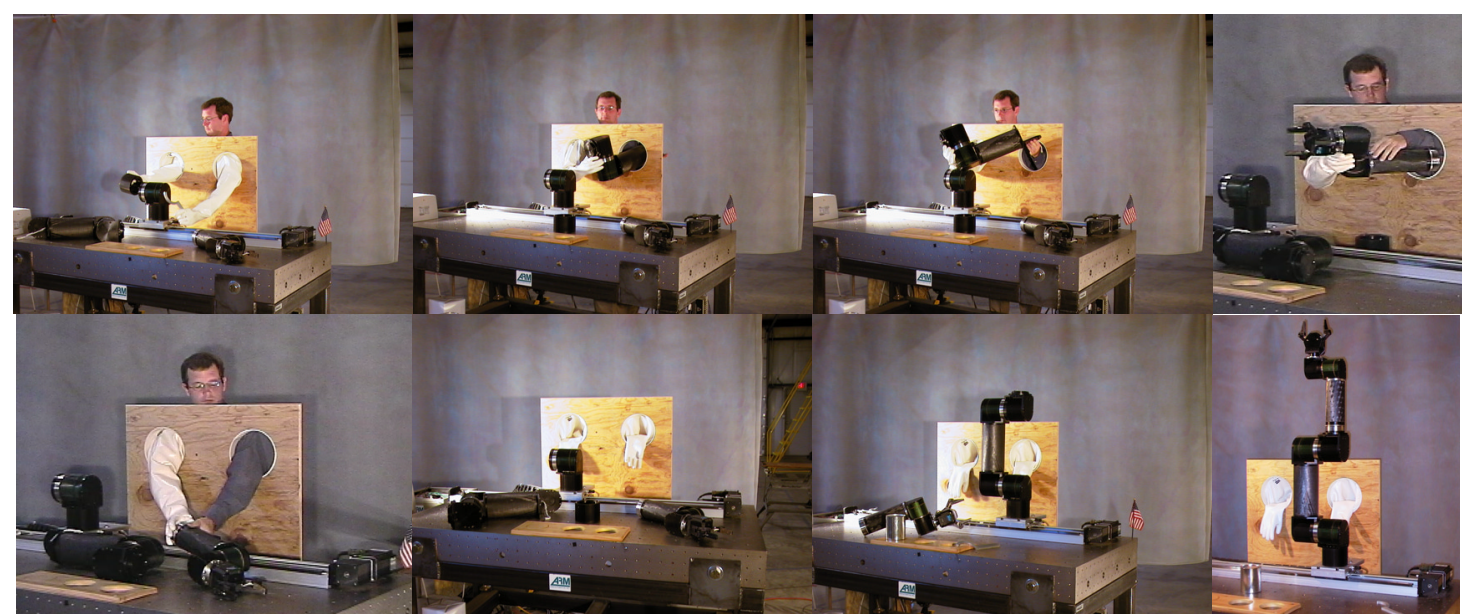

Figure 41: Large 4 DOF modular manipulator assembly sequence. 

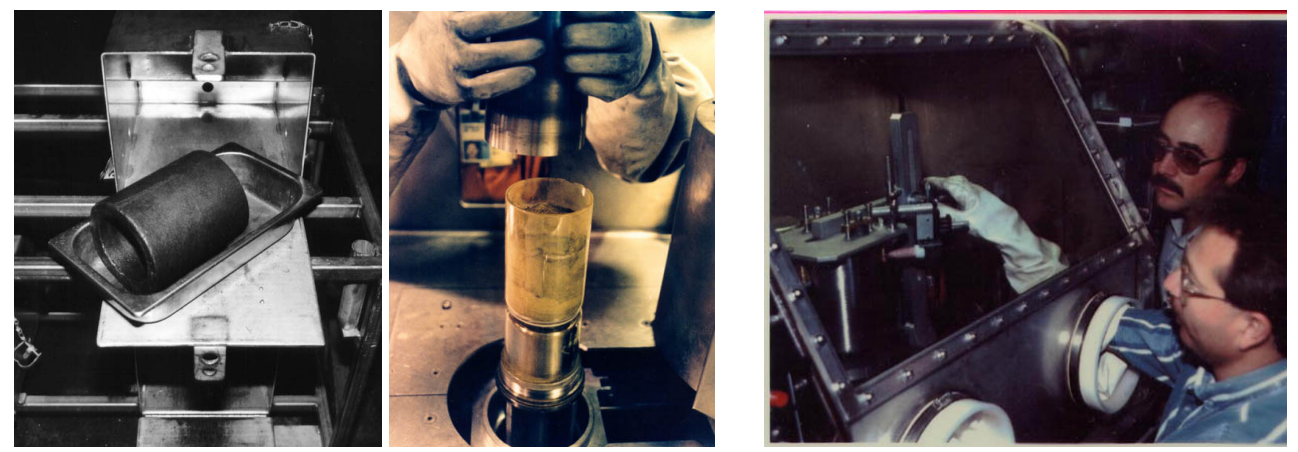

Figure 42: Insertion of MOX (lft) into convenience can (ctr) and primary can (rt) [MD, 2001].

\subsubsection{Assembly of a Convenience Can Inside a Primary Can}

There are several procedures associated with processing of pure Plutonium into a MOX fuel. Figure 42 demonstrates several of these tasks. A simple demonstration of the capabilities of the modular manipulator developed under this project is the assembly of a convenience can inside a stainless steel primary can. A convenience can is a hermetically sealed can that contains Plutonium that has been converted to a MOX fuel. The convenience can, when full, weighs approximately $4.8 \mathrm{~kg}$ and the complete assembly weights $5.1 \mathrm{~kg}$. For the demonstration, the clearance for the convenience can inside the primary can is less than $1.2 \mathrm{~mm}$ and the clearance for the lid on the primary can is less than $0.55 \mathrm{~mm}$.

Two demonstrations were performed, one with an empty convenience can using a 4 DOF system with the arm consisting of ARM20s and a 4 DOF system with the arm consisting of ARM32s for the lower two joints and an ARM20 for the distal joint. Figure 43 shows the sequence of the first manipulator assembling the canisters and then moving the complete assembly. Figure 44 shows the sequence of the second manipulator assembling the canisters and then moving the complete assembly. A jig was manufactured for positioning the convenience container, primary container and lid. This test not only demonstrates the repeatability of the manipulator but also the agility of the kinematics of this configuration.

\section{CONCLUSION}

Conclusions drawn on this project fall under two categories: 1) Baseline Technology Comparison, and 2) Commercial viability of modular robotics within the DOE Complex. Each of these issues will be discussed in the following subsections.

\subsection{Technology Advancements}

The successful demonstration of the modular technology developed under this project is a significant engineering feat. However, at its current state, the technology is not such that one can purchase actuators off-the-shelf and build one's own manipulator. The very fact that a modular system can be tailored to a task means that each system will always have a certain degree of customization. As such, it is difficult to directly compare this technology to the baseline of the LR-Mate 100i. Nonetheless, five technology advancements that were stated in the beginning will be addressed herein: 1) Increased net payload to weight ratio; 2) Smaller umbilical cords; 3) Capacity for customization; 4) Easily repairable or replaceable components; and 5) 


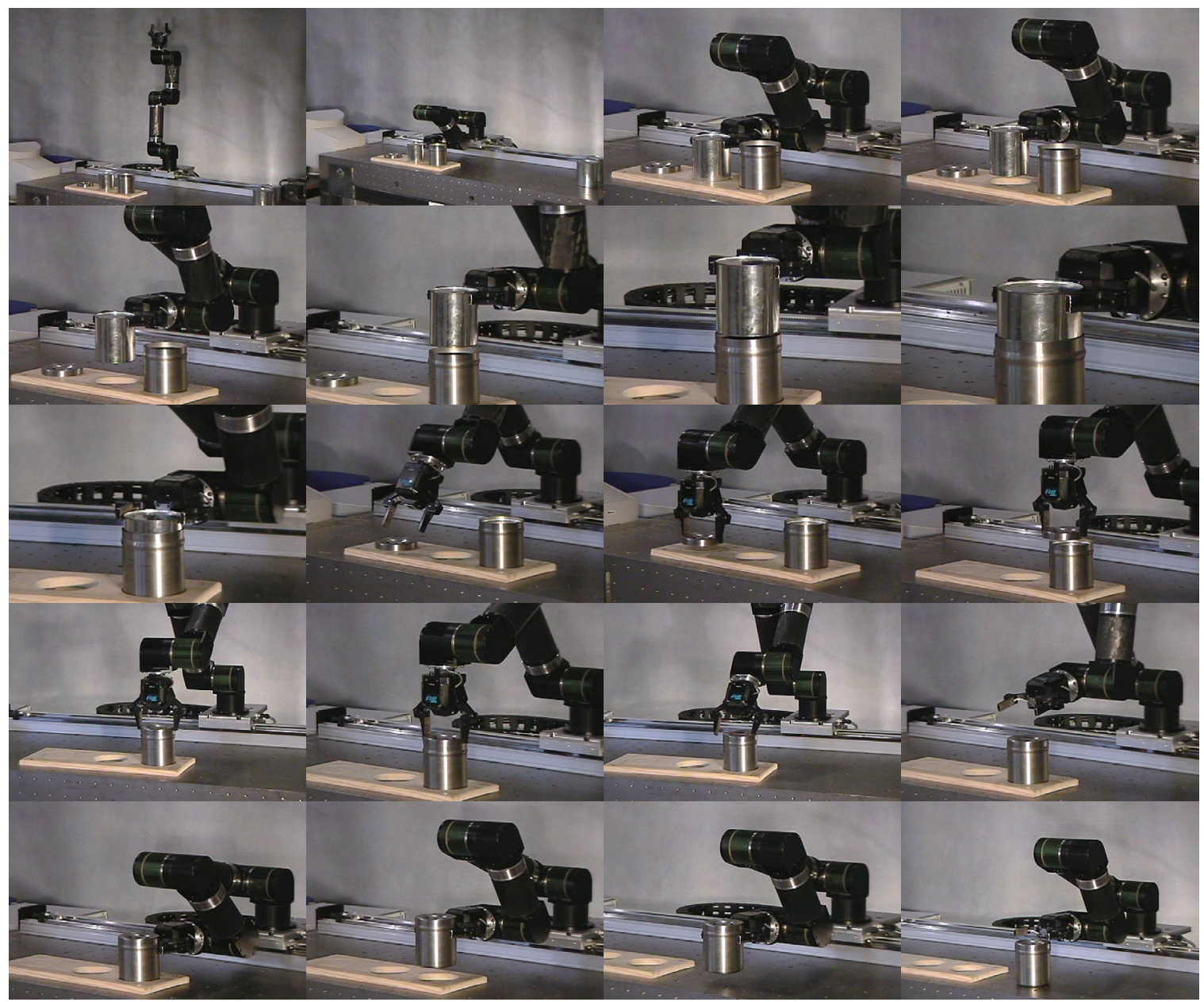

Figure 43: Smaller modular manipulator assembling Plutonium canisters.

Reconfigurable. Table 22 compares the baseline system to the two manipulators constructed for the demonstrations detailed herein.

The payload for the LR-Mate $100 i$ is specified $3 \mathrm{~kg}$ wrist payload with a maximum wrist moment of $55.5 \mathrm{~kg}_{\mathrm{f}} \mathrm{cm}$ about axis 4 . Thus, the center of mass including the end effector is located $185 \mathrm{~mm}$ (7.28 in) from axis 4. The mass of LR-Mate $100 i$ is $32 \mathrm{~kg}$ yielding a 3:32 wrist payload to weight ratio which is less than 1:10. The system tested in Figure 43, consisting of a Linear Track - P - P - P where each pitch joint consisted of an ARM20, had a wrist payload-toweight ratio of $3 \mathrm{~kg}$ with a reach of $790 \mathrm{~mm}$ to the center point of mass for the gripper. This is comparable to that of $785 \mathrm{~mm}$ for the LR-Mate 100i. The modular manipulator's mass for this configuration is $13.1 \mathrm{~kg}$ excluding the mass of the linear track yielding a payload to weight ration of 3:13.1. While this does exceed the LR-Mate 100i, it is not a fair comparison since the limiting factor is actually the joint with the lowest torque capability relative to its tool frame. For the LR-Mate, it is specified as the wrist axis (axis 4) while for the modular ARM it is the ARM20 in the proximal pitch position. By replacing the lower two pitch joints with ARM32s and maintaining the same configuration (see Figure 38 (b)), the wrist payload and manipulator mass increases to $9.5 \mathrm{~kg}$ (in full extension) and $25.6 \mathrm{~kg}$, respectively. This yields a wrist payload to weight ratio of 1:2.7. The reach for this configuration is $960 \mathrm{~mm}$ to the end effector. In this 


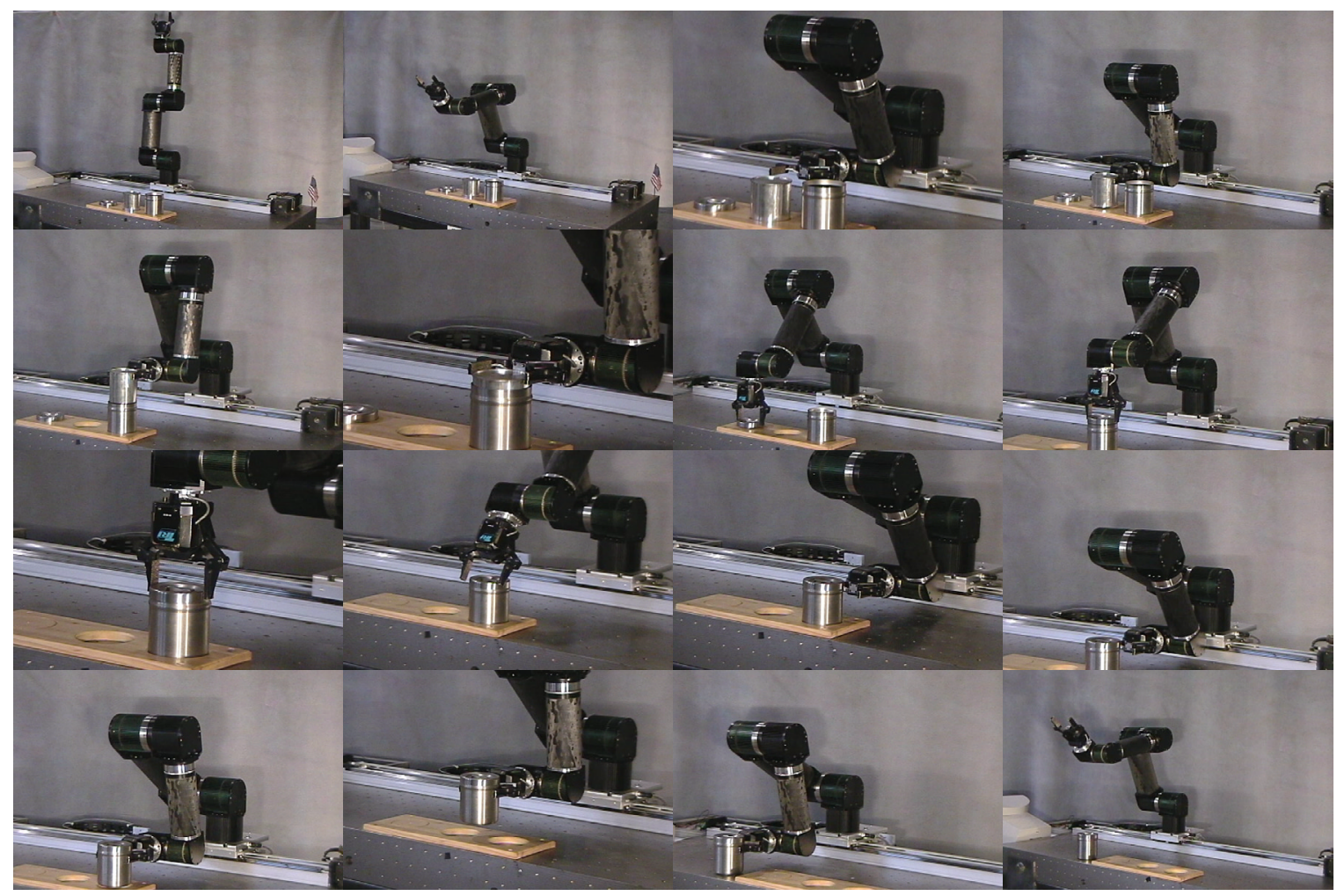

Figure 44: Larger modular manipulator assembling Plutonium canisters.

configuration, the limiting joint is the proximal ARM32. For reference, the mass of the linear track is $26.3 \mathrm{~kg}$. If the linear track is replaced with an ARM32 and mounting plate, the total manipulator mass becomes $34.58 \mathrm{~kg}$. Note that the effect that the manipulator \#2's reach is 960 $\mathrm{mm}$ versus $640 \mathrm{~mm}$ significantly reduces it effective wrist payload from $15.5 \mathrm{~kg}$ to $9.5 \mathrm{~kg}$.

The LR-Mate $100 i$ umbilical consists of 100 total wires, bundled into two 50-wire cables. One bundle is primarily power for the various components, and the other consists of command, control and sensing. These two wire cables are passed out of the glovebox using two fifty pin PAVE Technology feed-through connectors. These large cables placed inside a glovebox present access difficulties in being laid from the feed-through to the robot. In LANL instance, the umbilicals restrict access to several glove ports [Turner, 2001]. The umbilical for the modular robot is twelve wires regardless of the number of modules in the manipulator. This smaller umbilical associated with modular robotics will facilitate easier deployment of automation inside both new and existing glovebox facilities.

The LR-Mate $100 i$ robot has little capacity of customization other than, as options, one

\begin{tabular}{|l|c|c|c|}
\hline \multicolumn{1}{|c|}{ Item } & LR-Mate 100i & Manipulator \#1 & Manipulator \#2 \\
\hline Degrees of freedom & 5 & 4 & 4 \\
\hline Payload & $3 \mathrm{~kg}$ & $3 \mathrm{~kg}$ & $9.5 \mathrm{~kg}$ \\
\hline Mass of Modular Manipulator & $32 \mathrm{~kg}$ & $13.1 \mathrm{~kg}$ & $25.6 \mathrm{~kg}$ \\
\hline Mass with 1.2 m Track $(26.3 \mathrm{~kg})$ & $32 \mathrm{~kg}$ & $40.9 \mathrm{~kg}$ & $51.9 \mathrm{~kg}$ \\
\hline Mass with Base Roll & $32 \mathrm{~kg}$ & $18.3 \mathrm{~kg}$ & $34.58 \mathrm{~kg}$ \\
\hline Repeatability & $+/-0.04 \mathrm{~mm}$ & $+/-0.08 \mathrm{~mm}$ & $+/-0.08 \mathrm{~mm}$ \\
\hline Reach from base pitch to wrist plate & $620 \mathrm{~mm}$ & $640 \mathrm{~mm}$ & $960 \mathrm{~mm}$ \\
\hline
\end{tabular}

Table 22: Baseline and modular manipulator specifications. 

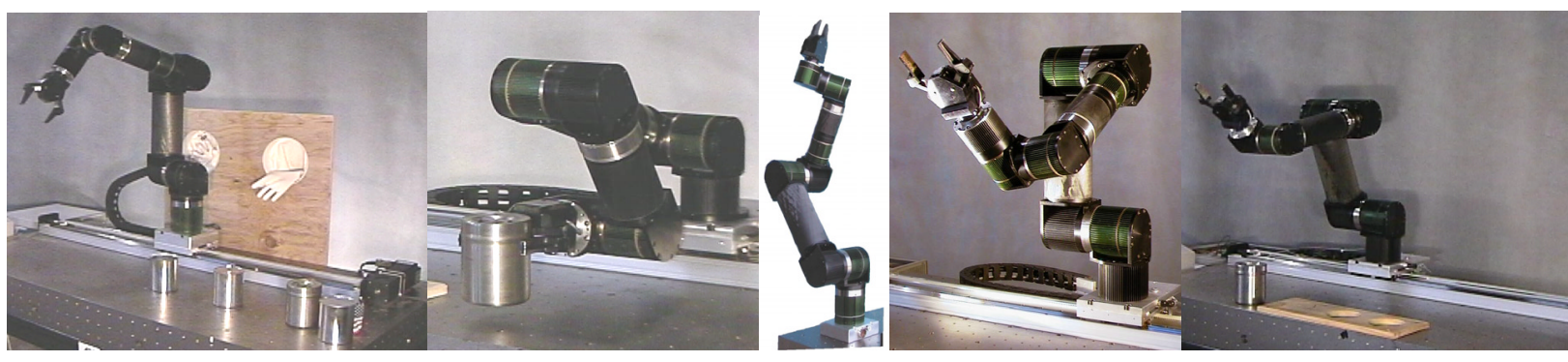

Figure 45: Various manipulator configurations comprised of ARM32 and ARM20 modules.

can increase the payload capacity at a cost of reducing the speed, and add a brake to joint 1 . The links in a modular robot can be fully customized to optimally meet the desired kinematics of an application. Additionally, with the incorporation of quick connects, a modular manipulator can be broken down and passed through an access port on the side of a glovebox. Figure 40 shows the process of three ARM20's being passed through a glove port and then assembled. Additionally, when the LR-Mate is inserted in the glovebox, it looses all of its configuration and homing parameters. These parameters have to be recalibrated inside the glovebox and this process a very time consuming task. This process is eliminated via the power-up homing technique employed by the DISC ${ }^{\mathrm{TM}} \mathrm{S}$ on the ARM modules. For glovebox automation, it becomes very important to match the workspace of the robot with the available area in the glovebox. With commercial robotics such as the LR-Mate 100i, the glovebox operations are designed for the robot, making it difficult to also accommodate manual operations [Turner, 2001].

With a pure custom robot, you design the robot for the glovebox, but only at the expense of time, and with the additional issues of unknown maintenance and reliability brought into play. With a modular robotic system, the maintenance and reliability histories of commercial parts can be utilized, while having considerable design freedom to produce a custom configuration. While this may not be as economical as purchasing a commercial robot straight up, it is certainly less costly than a purely custom design, and offers several significant advantages when compared to a commercial robot.

The LR-Mate being a monolithic manipulator is not reconfigurable for new or changing applications other than it can be mounted in either the upright or inverted position. In contrast, with just two base modules, ARM has configured a manipulator into at least 5 different configurations using these two modules. Figure 45 presents a sampling of these configurations. The issue of performing even the routine maintenance recommended by FANUC for the LRMate $100 i$ is a cause for concern.

\subsection{DOE Commercial Viability Of Modular Robotics}

The greatest benefit of automation and robotics comes in terms of worker safety. Any automation that removes humans from the risk of exposure provides a great and almost immeasurable service. As reviewed in Section 1.3, there are many DOE applications needing this technology included material processing in existing gloveboxes, characterization problems needing custom kinematics, and mixed waste sorting requiring low DOF. As demonstrated herein, the modular approach offers a viable solution for glovebox automation in new and existing systems that currently have no alternative but to use human labor. Still, this technology can be justified on this basis alone. Hence, one must present an argument justifying the 


\begin{tabular}{|c|r|r|r|}
\hline Description & Custom Robot & LR-Mate 100i & Modular Robot \\
\hline Initial Cost & $\$ 777,000$ & $\$ 50,000$ & $\$ 120,000$ \\
\hline Glovebox Customization & $\$ 9,360$ & $\$ 37,440$ & $\$ 9,360$ \\
\hline Place Manipulator in Glovebox & $\$ 936$ & $\$ 5,616$ & $\$ 936$ \\
\hline Calibrate Manipulator & $\$ 234$ & $\$ 1,872$ & $\$ 234$ \\
\hline Repair Manipulator Joint & $\$ 35,000$ & $\$ 50,000$ & $\$ 15,000$ \\
\hline Remove and Replace Joint & $\$ 1,872$ & $\$ 11,232$ & $\$ 1,872$ \\
\hline Place Manipulator in Glovebox & $\$ 936$ & $\$ 5,616$ & $\$ 936$ \\
\hline Calibrate Manipulator & $\$ 234$ & $\$ 1,872$ & $\$ 234$ \\
\hline Dispose of Broken Joint & $\$ 200$ & $\$ 15,000$ & $\$ 200$ \\
\hline Remove Broken Manipulator & $\$ 936$ & $\$ 5,616$ & $\$ 936$ \\
\hline Dispose of Broken Manipulator & $\$ 7,500$ & $\$ 15,000$ & $\$ 7,500$ \\
\hline Total Life Cycle Cost & $\underline{\mathbf{8 3 3 , 2 7 2}}$ & $\underline{\mathbf{\$ 1 9 3 , 6 4 8}}$ & $\underline{\mathbf{\$ 1 5 6 , 2 7 2}}$ \\
\hline
\end{tabular}

Table 23: Cost comparison of glovebox manipulators.

implementation of automation. This implementation must include the total life cycle costs associated with the automation in order to make a fair comparison between the use of personnel and automation. The technological advantages of modular robotics will decrease to an overall life cycle cost of a project by lowering the maintenance costs associated with a custom robot, decreasing timelines for customization of automation, etc. This section will present a comparison of the life cycle costs/savings against the baseline of the LR-Mate 100i and custom robotic systems.

\subsubsection{Methodology}

This analysis is based on calculating the estimated total life cycle costs for a custom robotic system, an LR Mate 100i, and a modular system. In presenting a life cycle cost comparison for glovebox manipulators, the following issues were taken into account: initial cost, glovebox customization, introduction of manipulator into glovebox, calibration of manipulator inside of glovebox, repair of manipulator inside of glovebox, disposal of broken joints and disposal of complete manipulator. Table 23 presents the estimated life cycle cost for a custom manipulator designed by the DOE personnel, a LR-Mate 100i and a 4 DOF modular system similar to that shown in Figure 38 (b). At the present time and state of the modular technology, the per unit reproduction costs for a P-R-R-R manipulator comprised of a linear track, two ARM32s and an ARM20 is estimated to resale at roughly $\$ 120,000$ depending upon the exact configuration which takes into account a degree of customization associated with a particular task. Major assumptions in arriving at these estimates are as follows:

- The materials for producing the custom manipulator cost $\$ 75,000$.

- The custom manipulator takes three man-years to design and build.

- The custom manipulator is of similar size to the modular manipulator.

- The LR-Mate is not repairable and the complete manipulator is disposable.

- The custom manipulator has similar specifications to the modular manipulator.

- All manipulators break at the same time. This is reasonable for the LR-Mate and modular manipulator since both use optical encoders which are lowest radiation resistant components in the system. If the custom manipulator has a high radiation tolerance, then its initial cost would be higher than the estimated time and materials. It is also assumed that only one joint fails and needs to be replaced. 
- The linear track is not disposed. Only the motor driving the linear track. The volume of the modular manipulator is less than half the LR-Mate. The custom designs volume is similar to that of the custom manipulator.

- The savings for removing DOE personnel is the same for all systems and not included in the overall system comparison.

Additionally, the cost comparison of introducing these manipulators to an existing glovebox cannot be made since the LR-Mate cannot be introduced into an existing glovebox due to the amount of customization required of the glovebox. Furthermore, the payload of the LR-Mate is not sufficient to perform many of the tasks involved with handling of MOX fuel.

\subsubsection{Cost Analysis}

\subsubsection{Estimated Life Cycle Costs Incurred}

There are many scenarios for implementation of these technologies. This analysis chose a very rudimentary approach. While the total life cycle costs associated with automation are much more complex than that presented herein, it does present a basis for comparing the technologies. The analysis below justifies the numbers presented in Table 23 .

Since the LR-Mate 100i is a monolithic device that can only be bagged in and out by a laborious procedure taking an estimated 6 man-days for removal, acquisition and installation, DOE personnel anticipate that a hot robot may be treated more as a disposable item, rather than a maintainable piece of equipment [McKee, 1998], [Turner, 2001]. A modular manipulator, on the other hand, can be broken down inside the glovebox and the failed joint removed through a simple procedure as shown in the previous section easily taking less than one-man day. Thus, only the broken component will require disposal as compared to a complete manipulator.

According to [Schaade, 1998] and [DOE, 2000c], the TRansUranic Waste (TRUW) disposal costs to the DOE sites per cubic meter range from $\$ 35,000$ to $\$ 100,000$ with a majority of the costs falling around $\$ 50,000$. The disposal volume of the LR-Mate is approximately 0.15 $\mathrm{m}^{3}$ and the disposal volume of an ARM module is $0.002 \mathrm{~m}^{3}$, thus the disposal cost will be 75 times less than that of the LR-Mate which is estimated to range from $\$ 5,250$ to $\$ 15,000$.

As commented by [Turner, 2001], the ability of modular robots to be customized to a task rather than the task being retrofitted to conform to the robot will save significant time. Additionally, unlike pure custom robotics, commercial repair parts will be readily available to maintain these systems. On the conservative side, it takes at least two to three man-years to develop a custom manipulator. Using the labor rate of $\$ 117 /$ man-hour as presented in [Dyches, et al, 1999] and a 2000 hour man-year, this means that a custom designed manipulator will cost the DOE between $\$ 468,000$ and $\$ 702,000$ plus the cost of materials. Additionally, the ability to purchase an off-the-shelf system versus building a custom manipulator, frees up DOE Site personnel to address the automation tasks rather than just the automation.

\subsubsection{Estimated Cost Reduction}

The nuclear industry estimates that it costs between \$2,000 [DOE, 1999] and \$10,000 [Bennett and Posey, 1997] per rem eliminated worker. The LANL Laboratory Procedure (LP) 107-16 provides the following guidelines for estimated person dosage costs for determining the cost savings for implementing ALARA principles: $\$ 2,000$ per rem-person dose if individual is below $2 / 3$ recommended amount of rem/year and $\$ 10,000$ per person-rem thereafter [LANL, 1998]. For purposes of drawing a cost comparison, this section will assume half the personnel 


\begin{tabular}{|c|c|c|c|}
\hline Plutonium Reduction Method & \# of Personnel & Max. Person-rem/yr & Est. Cost Reduction $^{1}$ \\
\hline LANL MOX Conversion & 120 & 150 & $\$ 450,000$ \\
\hline SRS Approach \#1 & 674 & 410 & $\$ 1,230,000$ \\
\hline SRS Approach \#2 & 991 & 561 & $\$ 1,683,000$ \\
\hline
\end{tabular}

Table 24: Estimated cost reduction for the baseline automation implementations.

will fall below and the other half will go over the $2 / 3$ recommended amount. This will effectively present a cost of approximately $\$ 6,000$ person-rem. This is a reasonable assumption since the required maximum annual dosage is presently set at 2 rem with anticipation of moving to $1 \mathrm{rem}$ in which case, all workers will their 2/3 maximum annual dose.

LANL estimates that the pit disassembly operation will expose annual average individual worker associated with the proposed action to an estimated at 750 mrem per year. They estimate this operation will require 120 individuals to complete the task resulting in 90 person-rem/year dose rate [DOE, 1998c]. Adding the pit disassembly dose rate estimate from SRS of 500 $\mathrm{mrem} /$ year, the LANL process will incur an additional $60 \mathrm{mrem}$-person/year in disassembling the pit. The SRS has proposed two approaches for disposing of the weapons grade plutonium: 1) Pit Disassembly and plutonium immobilization, and 2) Pit disassembly with a portion converted to MOX the remaining portion immobilized. The estimated dosages for the first method are 192 mrem-person/year and 218 mrem-person/year for pit disassembly and immobilization, respectively. This yields a total dosage of $410 \mathrm{mrem}$-person/year with an average worker dose of $618 \mathrm{mrem} /$ year. The estimated dosages for the second method are 192 mrem-person/year, $194 \mathrm{mrem}$-person/year and $175 \mathrm{mrem}$-person/year for pit disassembly, pit immobilization and MOX conversion, respectively. This yields a total dosage of $561 \mathrm{mrem}$ person/year with an average worker dose of 565 mrem/year ([DOE, 1997]). Table 24 summarizes the estimated cost reduction if half of these personnel were removed from the exposure.

\subsubsection{Return On Investment}

Each LANL MOX conversion demonstration line will require two manipulators: one for the pit disassembly and one for the canning and measuring process. LANL is anticipating using only a single line with two manipulators. The SRS approach \#1 calls for 2 lines to perform the pit disassembly and immobilization. It is anticipated with each line incorporating a gantry robot and two robots similar to the LR Mate. Approach \#2 calls 2 lines to perform the pit disassembly, MOX conversion, and immobilization. It is anticipated with each line incorporating a gantry robot and two robots similar to the LR Mate. Thus, Approach \#1 will utilize 4 manipulators and Approach \#2 will utilize 6 manipulators. Based on this analysis, all three automation solutions show a positive Return On Investment except for the single line at LANL. Table 25 summarizes the presentation presented herein. As this analysis shows, the modular technology achieves at least a $\$ 100,000$ in cost savings for almost all types of implementations over the baseline technology and can achieve a total savings in excess of $\$ 700 \mathrm{~K}$ if implemented on a large scale basis. 


\begin{tabular}{|c|r|r|r|}
\hline Plutonium Reduction Method & \multicolumn{1}{|c|}{ Est. Cost } & Est. Cost Reduction & Est. Savings/<Loss> \\
\hline \multicolumn{5}{|c|}{ Modular Manipulator } \\
\hline LANL MOX Conversion & $\$ 156,272$ & $\$ 450,000$ & $\$ 137,456$ \\
\hline SRS Approach \#1 & $\$ 625,088$ & $\$ 1,230,000$ & $\$ 604,912$ \\
\hline SRS Approach \#2 & $\$ 937,632$ & $\$ 1,683,000$ & $\$ 745,368$ \\
\hline \multicolumn{5}{|c|}{ Custom Robot } \\
\hline LANL MOX Conversion & $\$ 964,544$ & $\$ 450,000$ & $\$ 2,912$ \\
\hline SRS Approach \#1 & $\$ 1,227,088$ & $\$ 1,230,000$ & $\$ 193,368$ \\
\hline SRS Approach \#2 & $\$ 1,489,632$ & $\$ 1,683,000$ & $\$ 62,704$ \\
\hline \multicolumn{2}{|c|}{ LR-Mate $100 i$} \\
\hline LANL MOX Conversion & $\$ 193,648$ & $\$ 450,000$ & $\$ 455,408$ \\
\hline SRS Approach \#1 & $\$ 774,592$ & $\$ 1,230,000$ & $\$ 521,112$ \\
\hline SRS Approach \#2 & $\$ 1,161,888$ & $\$ 1,683,000$ & \\
\hline
\end{tabular}

Table 25: Estimated Return On Investment for the baseline automation implementations.

\subsubsection{Cost Conclusions}

As was demonstrated by this project, modular robotic technology can bring automation to existing gloveboxes reducing worker exposure and thereby reducing DOE's total cost. While it is difficult to estimate the total savings of reducing worker exposure, history has shown that automation within the DOE complex has proven to decrease worker accidents by decreasing their exposure [DOE, 2001]. The lines at LANL and SRS are excellent candidates to demonstrate this technology and prove that it is a viable and cost effective solution to the many DOE automation needs.

\section{FUTURE WORK}

The current commercial status of the modular robotics technology developed under this project is that it is ready for beta type testing. It is at the stage where an experienced engineer in the field of robotics can fully utilize the technology. The technology that still needs the most effort is the system level software. ARM had anticipated that the software purchased off-theshelf would be sufficient in controlling the manipulator. However, the interface between the system level software, which was designed for analog amplifiers, and the SERCOS communication system took more effort than anticipated. As a result, ARM has not significantly developed the error handling capability of the system level software. Another area of investigation is to get a better understanding of the radiation hardness of the system. ARM has done some preliminary investigation into the radiation hardness of some of the components but has not performed a full study of the complete system.

\subsection{Further System Level Testing}

Before the modules can become a commercial product that can be purchased off-theshelf, they will need to undergo more system level testing in real applications. Along that line, ARM is pursuing opportunities to advance the technology by getting systems into UT-RRG, 
LANL, and SNL for further testing. If funding is found to purchase a system by these groups, then outside testing by these knowledgeable personnel can be used to increase the overall reliability of these systems. This testing will be necessary to get the modular robotic technology approved by the Defense Nuclear Facilities Safety Board for glovebox use. Additionally, ARM is deploying this technology, under an SBIR Phase II award, for use in a remote site characterization application for D\&D at SRS. This effort will also further the overall reliability of the current system.

\subsection{Further Technology Developments}

There are several areas of the project that will need further study. The issues which need to be addressed in order to increase the overall system reliability are the error handling process of the system controller, the noise immunity of the communications signal, change out the brake material, implement a position loop in the DISC ${ }^{\text {TM }}$ and correct the windings on the ARM32.

The system controller presently handles all errors by completely shutting down the system which means removing power from the $\mathrm{DISC}^{\mathrm{TM}} \mathrm{S}$. The removal of power results in the loss of information regarding the cause of the error. An error handling parser needs to be written to determine the overall severity of the error and then determine if the system can recover from the error. The effort to fully implement such an error handler is estimated to take several man months.

The ARM20s can easily operate the main power bus at an excess of $340 \mathrm{~V}_{\mathrm{DC}}$ without any effects of noise but the ARM32 can currently function just $200 \mathrm{~V}_{\mathrm{DC}}$ before noise effects start to show up on the communications signals. The system is currently using an RS-485 line driver/receiver. ARM is considering changing this transmission technology to Low Voltage D Low Voltage Differential Signaling (LVDS) in an effort to overcome some of the shortcomings of the RS-485 communications medium.

Both the PSI and CCI boards had some very minor problems that need to be addressed. These issues are outlined for the CCI, PSI1 and PSI2 in their respective sections. These problems are presently corrected using jumper wires soldered externally on the board.

In order to incorporate a brake in the actuator design, ARM needed to pursue a custom design. The material selected for use as the frictional surface against the brake disk had a high initial coefficient of friction. ARM found that this coefficient degraded over time due to heat and wear. Additionally, there is no way to adjust the pressure applied by the brake after assembly to account for tolerances in the coefficient of friction. Both of these issues will need to be addressed in future redesigns.

A position loop with trajectory generation would be beneficial to the design of the system controller since the motion control board could then be eliminated. The DISC ${ }^{\mathrm{TM}} \mathrm{S}$ currently only have velocity and current control loops implemented. However, the DISC ${ }^{\mathrm{TM}} \mathrm{S}$ do have the SERCOS functional structure in place such that the position loop could easily be implemented.

The windings for the ARM32 were terminated with a high stress point where they are tied together and brought off of the motor. This has resulted in three of the original five ARM32s failing. Tying off the windings with more flexible wire under the coil bands and then bringing them off the stator can correct this rather than using the magnetic wire that is currently brought out. This will be implemented on the next redesign. 


\subsection{Radiation Hardening}

The radiation levels in many of the DOE applications range from a few $\mathrm{mRad} / \mathrm{hr}$ for filter change-out applications to up to approximately $8,000 \mathrm{Rad} / \mathrm{hr}$ in canyon remediation applications. For the most part, components for the modular actuators were selected such that a radiationhardened component could be substituted at a higher cost. A discussion below will cover some of the issues regarding radiation hardness of the components inside the modules. This discussion will be broken down into three sections: Mechanical Component Selection, wire selection, and electronics. Only the Total Ionizing Dose (TID) levels will be discussed. The data for the discussion presented below was obtained from [Bostock and Sias, 1994], [IR, 1998], [HP, 1984], [Bennett and Posey, 1997], and [NASA, 1996].

The mechanical components that are most effected by ionizing radiation are the seals, motor and solenoid windings, gear train (grease), and bearings. The seals used in the initial design of the modules are not radiation resistant although the company from which they were acquired does make seals that are radiation resistant to at least $100 \mathrm{MRad}$ (Si). These seals cost an order of magnitude greater than the commercially available seals used in the actuator. The polyethylene insulation on the motor and solenoid windings is good to at least $100 \mathrm{MRad}(\mathrm{Si})$ [Harper, 1997]. The grease used in the gear train is a commercial variant of an equivalent grease used by both the Aerospace and Space industries. The radiation tolerant grease can be used in place of the current grease at a higher cost and is good to about $10 \mathrm{MRad}$ (Si) [Harper, 1997]. The bearing's retaining rings and grease lubricants can be selected such that their radiation tolerance is increased. However, commercial grade bearings were selected to keep development costs down. The current devices with the nylon retainers are estimated to be good to approximately 1 MRad (Si) [Harper, 1997].

The wire insulation selected for the wire coil in the actuator was comprised of silicone rubber which is radiation tolerant to more than $10 \mathrm{MRad}$ (Si) [Harper, 1997]. This insulation also exhibited good lubrication qualities for the wire coil that was in torsion. The wire leads coming off the motor and sensors were coated in Teflon insulation and are good to about $30 \mathrm{Rad}$ (Si) [Bostock and Sias, 1994].

The electronics can be subdivided into two categories: Sensors and Controller. Since the DISC $^{\text {TM }}$ is enclosed inside an aluminum shell, Alpha and Beta radiation sources will have no effect on the degradation of its electronics. Consequently, only gamma radiation particles will have any effect on the life of the electronics. The sensors consist of Hall-Effect devices, thermistors, an RTD sensor, an optical encoder, a reflective optical sensor, and a switch. The switch is estimated to have a capacity to withstand at least $1 \mathrm{MRad}$ (Si). The Hall-Effect devices are CMOS based technology which is tolerant between the range of 3 to $50 \mathrm{KRad}$ (Si) [NASA, 1996], although according to [Bennett and Posey, 1997], they tend to range closer to $20 \mathrm{KRad}$ (Si). The thermistors and RTD sensor can withstand more than $1 \mathrm{MRad}(\mathrm{Si})$. According to properties of similar data taken from [HP, 1984], the encoder head should be tolerant to at least 3 $\mathrm{KRad}(\mathrm{Si})$ and components tested were tolerant to over $100 \mathrm{KRad}(\mathrm{Si})$.

The passives on the DISC ${ }^{\mathrm{TM}}$ consist of ceramic capacitors, metal film and carbon film resistors and silicon varistors of which all can withstand more than 1 e 8 Rad [Bennett and Posey, 1997]. The optocouplers on the board have a tolerance between 3 and $100 \mathrm{KRad}$ (Si). No data was found for the crystal oscillator but it is assumed to be at least as tolerant as the CMOS devices and [Bennett and Posey, 1997] has shown them to be at least $100 \mathrm{KRad}$ (Si). The microcontroller and digital logic on the CCI and PSI boards are CMOS based technology which 
technology is tolerant between the range of 3 to $50 \mathrm{KRad}$ (Si) [NASA, 1996], although according to [Bostock and Sias, 1994], they tend to range closer to $25 \mathrm{KRad}$ (Si). The DC-DC is not presently radiation-hardened but radiation-hardened versions with a TID of $50 \mathrm{KRad}$ (Si) or 100 KRad ( $\mathrm{Si}$ ) can be purchased. The transistors can be radiation-hardened to $1 \mathrm{MRad}$ ( $\mathrm{Si}$ ) [IR, 1998]. The transistor drivers are comprised of proprietary HVIC technology. No data is available on this technology, but it is comprised of Al-Si using large gates. It is therefore estimated to be at least tolerant to $10 \mathrm{KRad}(\mathrm{Si})$.

From the data presented herein, it is estimated that the $\mathrm{DISC}^{\mathrm{TM}} \mathrm{S}$ are tolerant to at least 3 $\mathrm{KRad}(\mathrm{Si})$ although conclusive results can only be obtained through actual testing. ARM is presently talking with Dr. Tulenko about performing future tests on the DISC ${ }^{\mathrm{TM}}$ electronics. This is the only way to arrive at any conclusive evidence as to exactly how radiation-tolerant the DISC $^{\mathrm{TM}} \mathrm{S}$ actually are.

\section{REFERENCES}

[ARIES 1998] U.S. Department of Energy (DOE). “ARIES Overview," Web Page, http://www.lanl.gov/aries/, 1998.

[ARIES, 2000] U.S. Department of Energy (DOE) NMFA. "Advanced Recovery and Integrated Extraction System (ARIES)," http://www.lanl.gov/orgs/esa/epe/ar_proj2000 aries.html, 2000.

[ARM, 1997] ARM Automation, Inc. “Accelerated Development Leading to Rapid Deployment of Cost Effective Modular Robotic Systems for Environmental Remediation,” April 1997.

[ARM, 2001] ARM Automation, Inc. “DISC "TM Description of SERCOS IDNs," 2001.

[Bajura, 2001] Bajura, R. A. "Memorandum: Environmental Management Call for Proposals to National Laboratories," DOE National Energy Technology Laboratory (NETL), February 2001.

[Bennett and Posey, 1997] Bennett, P. C. and L. D. Posey. "RHOBOT: Radiation Hardened Robotics,” SANDIA REPORT, SAND97-2405. October 1997.

[Black, 1998] Black, D. and Grupinski, S. "Evaluation of State-of-the-Art Manipulators and Requirements for DOE Robotics Applications." Topical Report, October 1998.

[BNFL, 1998] U.S. Department of Energy (DOE). "Rocky Flats Pu Stabilization System Behind Schedule, Over Budget," Weapons Complex Monitor, May 25, 1998, pp.5-6.

[BNFL, 1996] Uranium Institute Information Service. “UI News Briefing 96/13." Web Page, http://www.uilondon.org/nb/nb96/nb9613.htm, April 1996.

[Bostock and Sias, 1994] Bostock, J. L. and F. R. Sias. "Radiation Hardening for Terrestrial Robots For An Intelligent Inspection and Survey Robot," Report Prepared for the DOE under Contract No. DE-AC21-92MC29115. April 1994.

[Bronson, et al, 1997] Bronson, M., Ebbinghaus, B., Armantrout, G. and Gray. L. "Dealing with a Dangerous Surplus from the Cold War," Science and Technology Review. (pp. 5-13). Los Alamos, NM: Department of Energy, April 1997. 
[Burks, et al, 1997] Burks, B. L., D. D. Falter, R. L. Glassell, S. D. Van Hoesen, M. A. Johnson, P. D. Lloyd, and J. D. Randolph. "Development and Demonstration of a Remotely Operated Tank Waste Retrieval System for the Oak Ridge National Laboratory," ORNL9703, 1997.

[DDFA, 2000a] U.S. Department of Energy (DOE) D\&D Focus Area. “D\&D Focus Area Mission Statement," http://www.fetc.doe.gov/dd/, September 2000.

[DDFA, 2000b] U.S. Department of Energy (DOE) D\&D Focus Area. "Deactivation and Decommissioning Focus Area QUARTERLY REPORT - April - June 2000 Activities," Deactivation and Decommissioning Focus Area, July 2000.

[Dennison, et al, 1995] Dennison, D., R. L. Hurd, R. D. Merrill, and T. C. Reitz. "Application of Glovebox Robotics to Hazardous Waste Management," The ANS 6th Topical Meeting on Robotics and Remote Systems Conference Proceedings, Monterey, California, February 5-10, 1995.

[DOE, 1997] U.S. Department of Energy (DOE), Federal Register, "Surplus Plutonium Disposition Environmental Impact Statement," Notice of Intent, 62 FR 28009, Office of the Federal Register, Washington, DC, May 22, 1997.

[DOE, 1998a] U.S. Department of Energy (DOE). "Accelerating Cleanup: Path to Closure," DOE/EM-0362, June 1998.

[DOE, 1998b] U.S. Department of Energy (DOE). "Environmental Management Strategic Plan For Science and Technology," November 1998.

[DOE, 1998c] U.S. Department of Energy (DOE) Office of Fissile Materials Disposition. "Pit Disassembly and Conversion Demonstration Environmental Assessment and Research and Development Activities," Washington, DC, August 1998.

[DOE, 1999] U.S. Department of Energy (DOE) Industry Programs and Robotics Crosscutting Program. "Houdini TM-II Remotely Operated Vehicle System" DOE Document Number OST/TMS ID 2085. December 1999.

[DOE, 2000a] U.S. Department of Energy (DOE). "A Strategic Approach to Integrating the Long-Term Management of Nuclear Materials," DOE Report to Congress, June 2000.

[DOE, 2000b] U.S. Department of Energy (DOE). "DOE Environmental Quality R\&D Portfolio FY 1999-2001; Chapter 9: Deactivation and Decommissioning," February 2000.

[DOE, 2000c] U.S. Department of Energy (DOE). "DOE/AL Site Technology Coordinating Group FY-2000 Problem Statements: Los Alamos National Laboratory.” 2000.

[DOE, 2001] U.S. Department of Energy (DOE). "Tritium Facility Operation." http://www.srs.gov/general/srtech/tritweb/operations.htm, 2001.

[DP, 1996] U.S. Department of Energy (DOE) Office of Defense Programs. "Safety Evaluation Report of the Los Alamos National Laboratory Technical Area 55 Plutonium Facility 4 Safety Analysis Report and Technical Safety Requirements," December 1996. 
[Dyches, et al, 1999] Dyches, G., B. Fiscus, L. Hamilton, G. Hovis, R. Jones, E. Kriikku, B. Randall, M. Restivo, J. Steed, and C. Ward. "Plutonium Immobilization Can Loading FY99 Component Test Report," WSRC-TR-1999-00318, 1999.

[EM, 1998] U.S. Department of Energy (DOE). "Environmental Management Strategic Plan For Science And Technology," June 1998.

[EM, 2000] U.S. Department of Energy's Environmental Management Program. “Technology Development Statement," http://www.em.doe.gov/emprimer/techdev.html, May 2000.

[EPE, 2000] U.S. Department of Energy (DOE), ESA-EPE Group Office. "The MOVER System," EPE News, Vol. 1, No. 1, pg. 1. September 2000.

[Evans, 2000] Evans, J. M., National Institute of Standards and Technology (NIST). “Open Architecture Controls: The Key to Interoperability" Slide \# 2. Slide Presentation given during RIA Annual Forum, Orlando, FL. November 2000.

[FANUC, 1993] FANUC Robotics North America. "LR-Mate" product literature, 2000 South Adams Road, Auburn Hills, MI 48326-2800, (313) 377-7000, 2 pages, 1993.

[GNET, 1998] U.S. Department of Energy (DOE) National Energy Technology Laboratory and The Global Environment \& Technology Foundation. "Remote Automated Systems and Tooling," http://www.gnet.org/helpcenter/minfo.cfm, December 1998.

[Harper, 1997] Harper, C. A. "Electronic Packaging and Interconnection Handbook." $2^{\text {nd }}$ Edition. McGraw-Hill. 1997.

[Hollen and Rzeszutko, 1997] Hollen, R. and C. Rzeszutko. "Automating the Analytical Laboratory via the Chemical Analysis Automation (CAA) Paradigm," Los Alamos National Laboratory, LAUR-97-4023, pp. 5, and 7, 1997.

[HP, 1984] Hewlett Packard (HP). "Radiation Immunity of Hewlett-Packard Optocouplers." Application Note No. 1023, 1984.

[HP, 1994] Hewlett Packard (HP). "The ORCA System - Optimized Robot for Chemical Analysis," product brochure, 4 pages, March 1994.

[IEC, 1995] International Electrotechnical Commission (IEC). "Electrical Equipment of Industrial Machines - Serial Data Link for Real-Time Communication Between Controls and Drives" IEC Std. No. 1491, First Edition. 1995.

[IR, 1998] International Rectifier (IR). “International Rectifier's Total Dose Radiation Hardness Assurance (RHA) Test Program.” Application Note No. 999, 1998.

[Kiebel, 1997] Kiebel, G. R. "Light Duty Utility Arm Deployment in Hanford Tank T-106". PNNL-11635, Pacific Northwest National Laboratory, Richland, Washington, 1997.

[Kriikku, et al, 1998] Kriikku, E., and J. Brault. "Plutonium Immobilization - Puck Handling," WSRC-MS-98-00897, 1998.

[Kriikku, et al, 1999] Kriikku, E., and G. Hovis. "Plutonium Immobilization - Can Loading," WSRC-TR-99-00318, 1999. 
[LANL, 1998] Los Alamos National Laboratory. "The Actinide Research: Nuclear Materials Research and Technology Quarterly", $1^{\text {st }}$ Quarter, 1998.

[Lyman, 1998] Lyman, E. S., Department of Energy (DOE). "DOE reprocessing Policy and The Irreversibility of Plutonium Disposition,” Nuclear Control Institute, April 1998.

[McKee, 1998] McKee, R. Conversations with Randy McKee during site visit to Sandia National Labs, June 18, 1998.

[MD, 2001] U.S. Department of Energy (DOE) National Energy Technology Laboratory Material Deposition Focus Area. "Pu Photo Gallery - Pit Disassembly and Conversion", "http://www.esa.lanl.gov/groups/esaepe/aries.html, 2001.

[MFM, 1998] MFM Technology, Inc. “Motion Control Selection Guide,” Product Literature, 200-13 Avenue, Ronkonkoma, NY 11779, (800) 636-6867, 1998.

[NASA, 1996] National Aeronautical Space Agency (NASA). "Space Radiation Effects On Electronic Components In Low-Earth Orbit." Preferred Reliability Practices: Practice NO. PD-ED-1258, April 1996.

[NMFA, 1999] U.S. Department of Energy (DOE) NMFA. "Nuclear Materials Focus Area FY 2000 - FY 2004 Multi-Year Program Plan,” DOE/ID-10728, November 1999.

[NMFA, 2000a] U.S. Department of Energy (DOE) NMFA. "NMFA July 2000 Monthly Report". July 2000.

[NMFA, 2000b] U.S. Department of Energy (DOE) NMFA. "Nuclear Materials Focus Area FY 2000 Annual Report," , November 2000.

[NMFA, 2000c] U.S. Department of Energy (DOE) NMFA. "Request for Proposals to Support the Nuclear Materials Focus Area (NMFA) Fiscal Year (FY) 2001 Technical Program Attachment 2: Scope of Work for FY2001 NMFA Call for Proposals," July 2000.

[Noakes, et al, 1997] Noakes, M. W., Haley, D. C., and Willis. W. D. "The selective equipment removal system dual arm work module," ANS Proceedings of the 7th Topical Meeting on Robotics \& Remote Systems (pp. 478-483). LaGrange Park, IL: American Nuclear Society, 1997.

[OST, 1999] U.S. Department of Energy (DOE) Office of Science and Technology. "Environmental Management Office of Science and Technology Management Plan," February 1999.

[Parker, 1996] Parker Hannifin Corporation. "SM Series Brushless Servo Motors," Product Literature, 5500 Business Park Drive, Rohnert Park, CA 94928, (800) 358-9070, July 1996.

[RFCAB, 2000] Rocky Flats Citizens Advisory Board. "Rocky Flats Cleanup Progress Report," Autumn 2000 Issue, September 2000.

[RIA, 1999] Robotic Industries Association (RIA)/American National Standards Institute, Inc. (NIST). "American National Standard for Industrial Robots and Robot Systems - Safety Requirements" 1999.

[Richardson, 2000] Richardson, et al. “Long-Term Stewardship Study Draft,” October 2000. 
[RIM, 1998] U.S. Department of Energy (DOE) RIM. "Robotics And Intelligent Machines In The U.S. Department Of Energy: A Critical Technology Roadmap," SANDIA REPORT, SAND98-2401. October 1998.

[RIM, 2000] U.S. Department of Energy (DOE). "The Robotics and Intelligent Machines (RIM) Initiative At The U.S. Department Of Energy: Basis," Web Page. http://www.rim.doe.gov/documents/WhatisRIM.html, 2000.

[Roberson, 1998] Roberson, J. "Re: Turnover of the Plutonium Stabilization and Packaging System”, Memorandum. U.S. Department of Energy RFFO, September 1998.

[Rocky Flats, 1998] U.S. Department of Energy (DOE). "Rocky Flats Closure Project Management Plan,” pp. 4-2, June 1998.

[Schaade, 1998] Schaade, J. "FY98 Accomplishments - Nuclear Materials Stabilization," Presentation, 1998.

[Schilling, 1999] ALSTOM Automation Schilling Robotics. "The TITAN 3 Remote Manipulator System," Product Brochure. 201 Cousteau Place, Davis, California 95616, (530) 753-6718, 1999.

[SERCOS, 1998a] Interests Group SERCOS Interface e. V. "SERCOS Interface: Update 98.1" Update to IEC Std. No. 6-1491. 1998.

[SERCOS, 1998b] Interests Group SERCOS Interface e. V. "SERCOS Interface: Description of Indentnumbers" Supplement to IEC Std. No. 6-1491. 1998.

[Tesar, et al, 2001] Tesar, D., D. Cox, C. Kapoor, and S. Kang. "Test Result of Modular Actuator (ARM20) Using Actuator Endurance \& Reliability Test Bed," The University of Texas At Austin Robotics Research Group, 2001.

[Tibrea and Maddux, 1998] Tibrea, S. L. and, P. E. Maddux. "Proposed New DOE Remote Facilities Overview - Designing for the Next Century," Westinghouse Savannah River Company - Savannah River Site, WSRC-MS-98-00571, 1998.

[Turner, 2001] Turner, C. Email from Cameron Turner regarding glovebox automation at Los Alamos National Labs, July 9, 2001. 


\section{LIST OF ACRONYMS AND ABBREVIATIONS}

$\begin{array}{ll}\text { A } & \text { Amp } \\ \text { ALARA } & \text { As Low As is Reasonably Achievable } \\ \text { ANL } & \text { Argonne National Laboratory } \\ \text { API } & \text { Application Programming Interface } \\ \text { APP } & \text { Automated Plutonium Processing } \\ \text { ARIES } & \text { Advanced Recovery and Integrated Extraction System } \\ \text { ARM } & \text { ARM Automation, Inc. } \\ \text { ARM20 } & \text { Small Actuator designed under DOE contract } \\ \text { ARM32 } & \text { Large Actuator designed under DOE contract } \\ \text { AT } & \text { Amplifier Telegram } \\ \text { AtoD } & \text { analog to digital } \\ \text { BDM } & \text { Background Debugger Mode } \\ \text { CAD } & \text { Computer Aided Drafting } \\ \text { CAN } & \text { Controller Area Network } \\ \text { CCI } & \text { Control and Communications Interface } \\ \text { CCW } & \text { Counter-Clock Wise } \\ \text { CE } & \text { Conformité Européenne } \\ \text { CFM } & \text { Cubic Feet Per Minute } \\ \text { CMST } & \text { Characterization, Monitoring \& Sensor Technology } \\ \text { CODE } & \text { Cimetrix Open Development Environment } \\ \text { CP } & \text { Communication Phase } \\ \text { CPLD } & \text { Complex Programmable Logic Device } \\ \text { CPU } & \text { Central Processing Unit } \\ \text { D\&D } & \text { Deactivation \& Decommissioning } \\ \text { DAC } & \text { Data Acquisition } \\ \text { DC } & \text { Direct Current } \\ \text { DH } & \text { Denavit-Hartenberg } \\ \text { DISC } & \text { Distributed Intelligent Servo Controller } \\ \text { DOE } & \text { Department of Energy } \\ \text { DOF } & \text { Degrees of Freedom } \\ \text { DP } & \text { Defense Programs } \\ \text { DSP } & \text { Digital Signal Processor } \\ \text { DWPF } & \text { Defense Waste Processing Facility } \\ \text { EEPROM } & \text { Electrically Erasable/Programmable ROM } \\ \text { EM } & \text { Environment Management } \\ \text { EMF } & \text { ElectroMotive Force } \\ \text { EMI } & \text { ElectroMagnetic Interference } \\ \text { ESP } & \text { Efficient Separations and Processing } \\ \text { E-Stop } & \text { Emergency Stop } \\ \text { FA } & \text { Focus Areas } \\ \text { FFT } & \text { Fast Fourier Transform } \\ \text { FSMA } & \text { Fiber Sub Miniature Assembly } \\ \text { FY } & \text { Fiscal Year (Government) } \\ & \end{array}$




\begin{tabular}{|c|c|}
\hline HAN & Hanford Complex \\
\hline $\mathrm{I} / \mathrm{O}$ & Input/Output \\
\hline IBM & International Business Machines \\
\hline IDE & Integrated Drive Electronics \\
\hline IDN & SERCOS Identification Number \\
\hline INEEL & Idaho National Engineering and Environmental Laboratory \\
\hline IP & Industry Programs \\
\hline Kbytes & Kilo-Bytes \\
\hline $\mathrm{kg}$ & Kilogram \\
\hline $\mathrm{kHz}$ & Kilo-Hertz \\
\hline LANL & Los Alamos National Laboratory \\
\hline $1 b_{\mathrm{m}}$ & Pound Mass \\
\hline LED & Light Emitting Diode \\
\hline LLNL & Lawrence Livermore National Laboratory \\
\hline LVDS & Low Voltage D Low Voltage Differential Signaling \\
\hline $\mathrm{m}$ & meter \\
\hline $\mathrm{mm}$ & millimeter \\
\hline $\mathrm{mA}$ & milliamp \\
\hline $\mathrm{MC}$ & Momentarily Closed \\
\hline MD & Materials Disposition \\
\hline MDT & Master Data Telegram \\
\hline MEI & Motion Engineering, Inc. \\
\hline $\mathrm{MHz}$ & Megahertz \\
\hline MLUDA & Modified Light Duty Utility Arm \\
\hline MOX & Metal Oxide \\
\hline $\mathrm{MPa}$ & MegaPascals \\
\hline MTBF & Mean Time Between Failure \\
\hline MW & Mixed Waste \\
\hline $\mathrm{N}$ & Newton \\
\hline $\mathrm{N} / \mathrm{A}$ & Not Applicable \\
\hline NASA & National Aeronautical Space Agency \\
\hline $\mathrm{NC}$ & Normally Closed \\
\hline NDA & Non-Destructive Assay \\
\hline NM & Nuclear Materials \\
\hline NO & Normally Open \\
\hline$\Omega$ & $\mathrm{Ohm}$ \\
\hline OSCAR & Operational Software Components for Advanced Robots \\
\hline OST & Office of Science and Technology \\
\hline $\mathrm{OZ}$ & Ounces \\
\hline $\mathrm{P}$ & Pitch \\
\hline $\mathrm{PC}$ & Personal Computer \\
\hline PCB & Printed Circuit Board \\
\hline PID & Proportional-Integral-Derivative \\
\hline P-IDN & Product Specific IDN \\
\hline PIP & Plutonium Immobilization Project \\
\hline
\end{tabular}




$\begin{array}{ll}\text { PLL } & \text { Phase Lock Loop } \\ \text { PSI } & \text { Power and Sensor Interface } \\ \text { PSI } & \text { Pounds per Square Inch } \\ \text { PSI20 } & \text { Small actuator's Power and Sensor Interface board } \\ \text { PSI32 } & \text { Large actuator's Power and Sensor Interface board } \\ \text { PSSCI } & \text { Power Supply and System Controller Interface } \\ \text { Pu } & \text { Plutonium } \\ \text { PuSPS } & \text { Plutonium Stabilization and Packaging Systems } \\ \text { PWM } & \text { Pulse Width Modulation } \\ \text { QC } & \text { Quick Connect } \\ \text { R } & \text { Roll } \\ \text { rad } & \text { Radian } \\ \text { RAM } & \text { Random Access Memory } \\ \text { Rem } & \text { roentgen equivalent man } \\ \text { RFETS } & \text { Rocky Flats Environmental Technology Site } \\ \text { RIM } & \text { Robotics In Manufacturing } \\ \text { RMS } & \text { Root Mean Square } \\ \text { ROM } & \text { Read Only Memory } \\ \text { RPM } & \text { Revolutions Per Minute } \\ \text { RTD } & \text { Resistance Temperature Detector } \\ \text { SERCOS } & \text { SErial Real-time COmmunications System } \\ \text { S-IDN } & \text { SERCOS specific IDN } \\ \text { SMT } & \text { Surface Mount } \\ \text { SNL } & \text { Sandia National Laboratory } \\ \text { SRAM } & \text { Static RAM } \\ \text { SRS } & \text { Savannah River Site } \\ \text { SSI/SPI } & \text { Synchronous Serial Interface/Serial Peripheral Interface } \\ \text { START } & \text { Strategic Arms Reduction Treaty } \\ \text { STCG } & \text { Site Technology Coordination Group } \\ \text { SVGA } & \text { Super Video Graphics Array } \\ \text { TCP/IP } & \text { Transmission Control Protocol/Internet Protocol } \\ \text { TID } & \text { Total Ionizing Dose } \\ \text { TRUW } & \text { TRansUranic Waste } \\ \text { TWR } & \text { Tank Waste Retrieval } \\ \text { UT } & \text { The University of Texas at Austin } \\ \text { UTRRG } & \text { UT Robotics Research Group } \\ \text { V } & \text { Volt } \\ \text { V } \text { AC } & \text { Volts, Alternating Current } \\ \text { W } & \text { Watt } \\ \text { WIPP } & \text { Waste Isolation Pilot Plant }\end{array}$

